PHYSICAL MODEL TESTING OF AN EXPERIMENTAL VORTEX DEVICE FOR CONTROLLING TOTAL SUSPENDED SOLIDS IN STORMWATER

\author{
by \\ Zihan Mi \\ B.Eng, Yunnan Agricultural University, China, 2017
}

\author{
An MRP \\ presented to Ryerson University \\ in partial fulfillment of the \\ requirements for the degree of \\ Master of Engineering \\ in the program of \\ Civil Engineering \\ Toronto, Ontario, Canada, 2020 \\ (C) Zihan Mi, 2020
}




\section{Author's Declaration}

I hereby declare that I am the sole author of this MRP. This is a true copy of the MRP, including any required final revisions.

I authorize Ryerson University to lend this MRP to other institutions or individuals for the purpose of scholarly research.

I further authorize Ryerson University to reproduce this MRP by photocopying or by other means, in total or in part, at the request of other institutions or individuals for the purpose of scholarly research.

I understand that my MRP may be made electronically available to the public. 


\title{
Physical Model Testing of an Experimental Vortex Device for Controlling Total Suspended Solids in Stormwater
}

\author{
Zihan Mi \\ Master of Engineering, 2020 \\ Civil Engineering, Ryerson University
}

\begin{abstract}
In highly urbanized area, lack of space limits the application of most stormwater quality treatment technologies. Oil/Grit Separators (OGSs) are preferred in these cases due to their compact size and reasonable solids removal efficiency. The objective of this research is to identify the challenges and practical potential solutions of solids treatment performance testing on a full-scaled experimental vortex device (EVD) adopting TRCA's regulatory guideline titled the "Procedure for Laboratory Testing of Oil/Grit Separators" (referred to in this paper as the Procedure) which stipulates the standards of sediments and oil removal tests in Canada. The test results indicated that: (1) TSS treatment efficiency of EVD was observed to decline with the particle size and flow rate; and (2) the average overall TSS treatment efficiency decreased from $52 \%$ to $44 \%$ as the flow rate doubled.
\end{abstract}




\section{Acknowledgements}

I would like to take this opportunity to thank a multitude of people who have supported me throughout my graduate study and all along.

Firstly, I would like to express my sincere gratitude to my supervisor Dr. James Li, who has offered great guidance and generous support to me with considerate encouragements.

My thank also extends to my instructor Dr. Darko Joksimovic and aunt Dr. Weihua Cao for their superior inspiration and guidance in my project.

Furthermore, I wish to especially acknowledge the help and funding support provided by the Natural Science and Engineering Research Council and Nankai University, as well as the technical support by staffs at the Chinese Communications Construction Inc. for my experimental testing. 


\section{Dedication}

To the ones I care the most, who always have faith in me, support me without any doubt, comfort me when difficult times arrive, guard my dreams with all the strength, and love me as much as I love them. I believe here and now is a perfect chance to let you know that, Mingming, Pangzi, and Ranran, you are the light of my life. 


\section{Table of Contents}

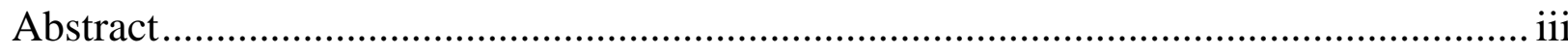

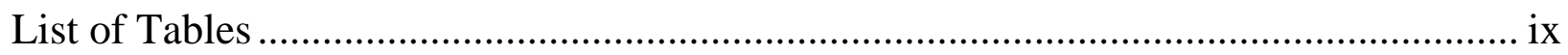

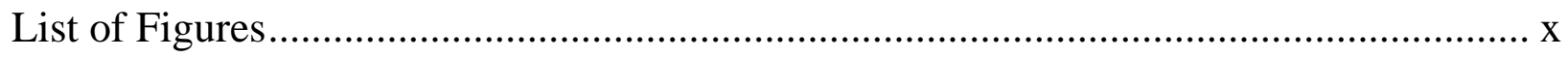

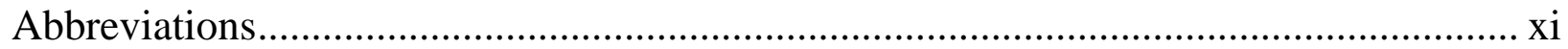

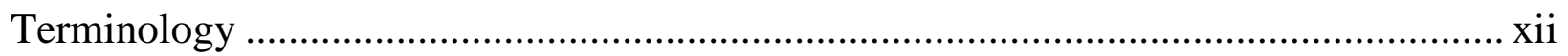

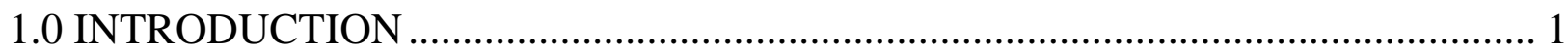

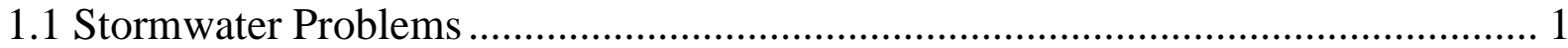

1.2 Stormwater Management Development............................................................... 2

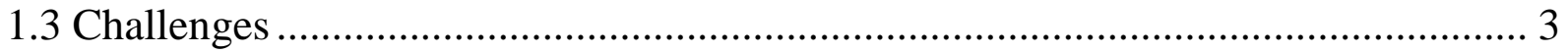

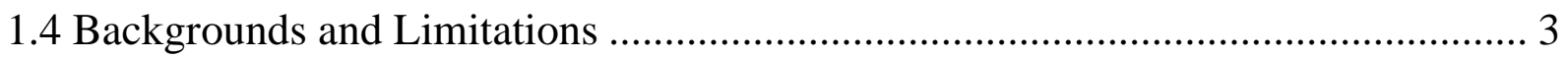

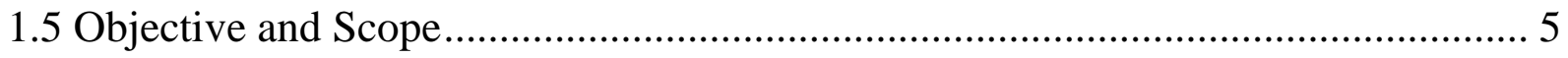

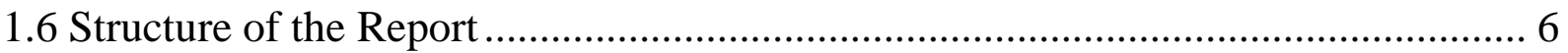

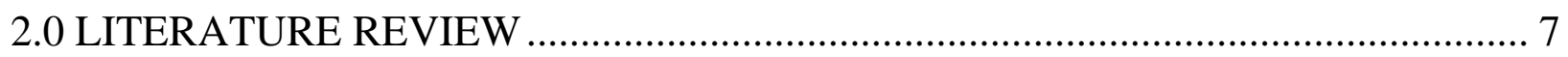

2.1 Review of Hydrodynamic Vortex Separators ……................................................... 7

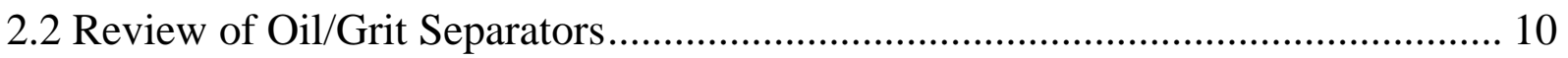

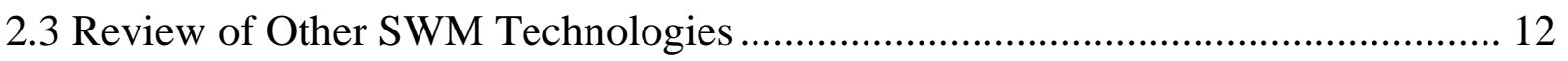

2.4 Physical Hydraulic Modeling ............................................................................ 12

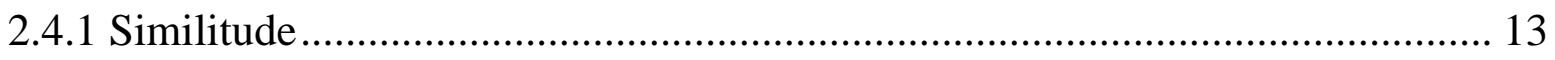

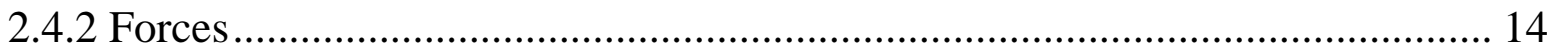

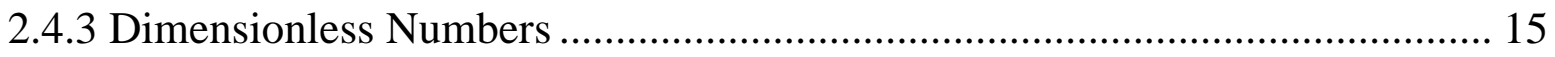

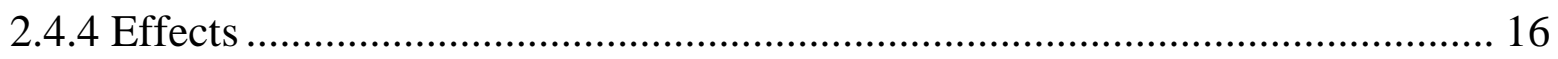

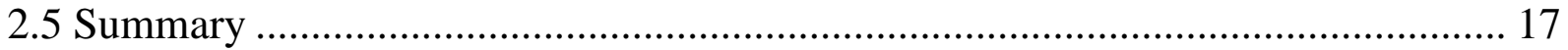

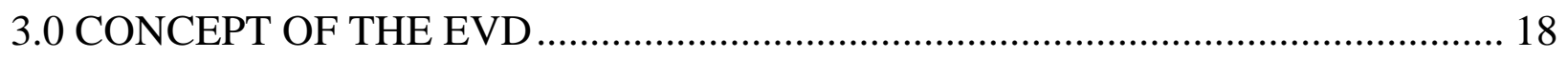

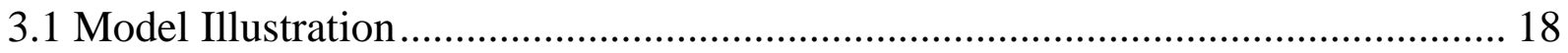

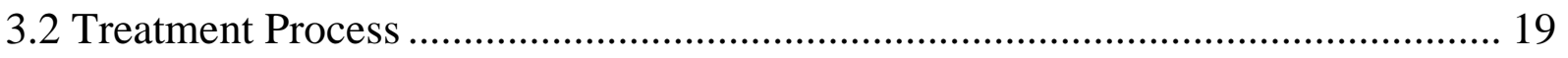

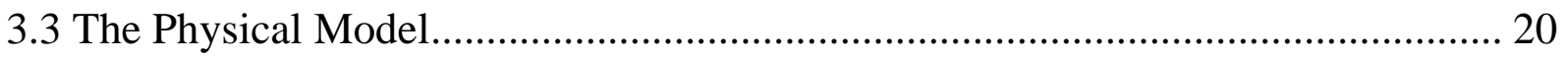




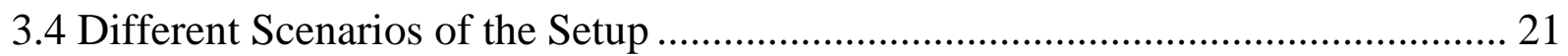

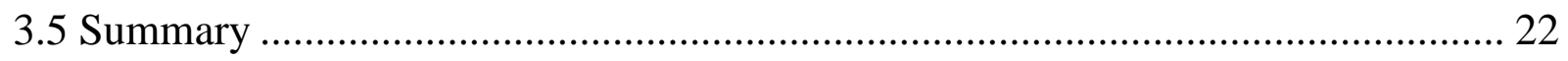

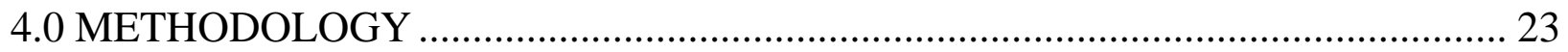

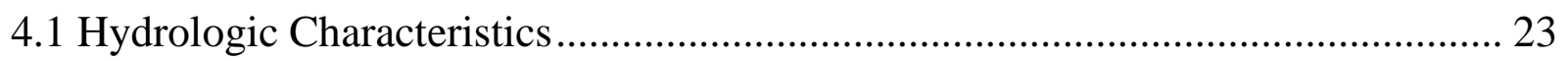

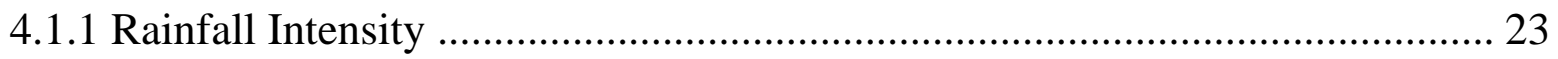

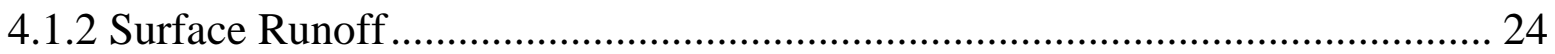

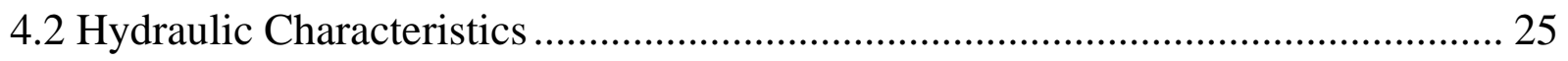

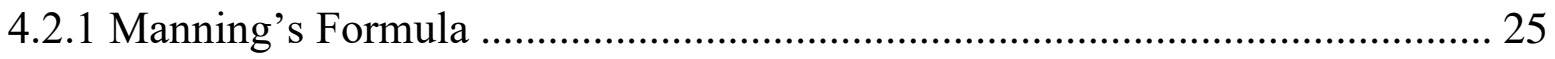

4.2.2 On-Site Layout and Profile _................................................................................ 26

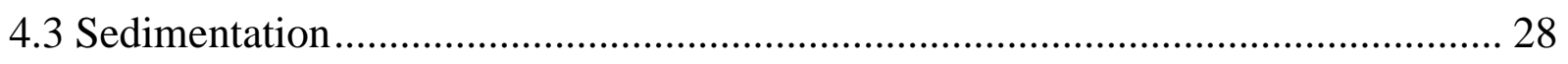

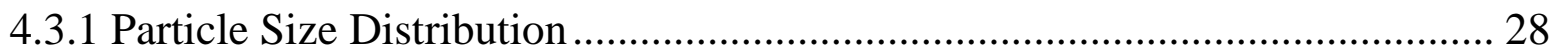

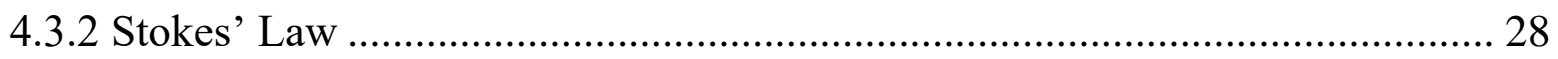

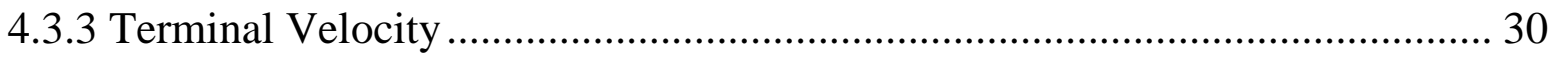

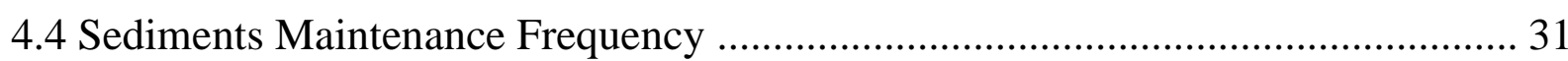

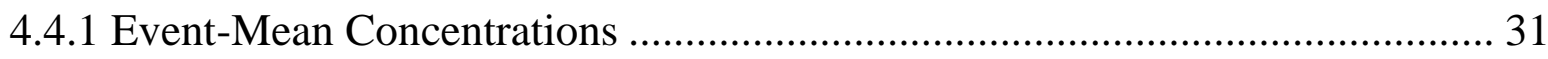

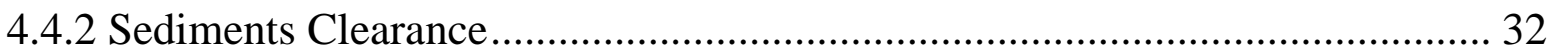

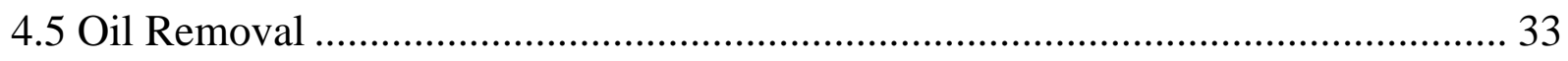

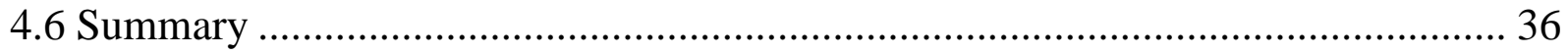

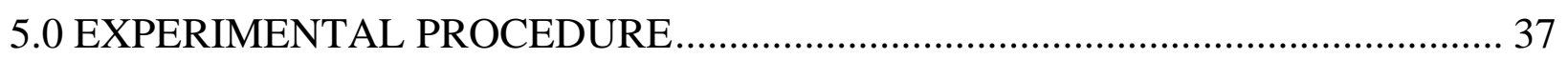

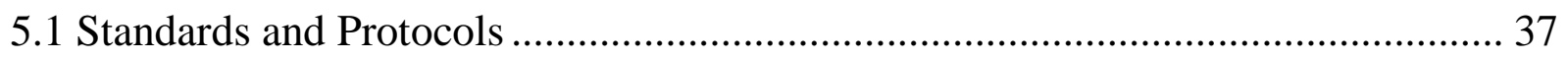

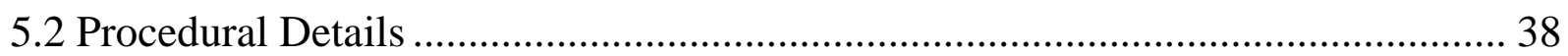

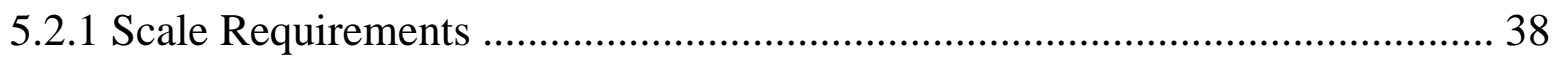

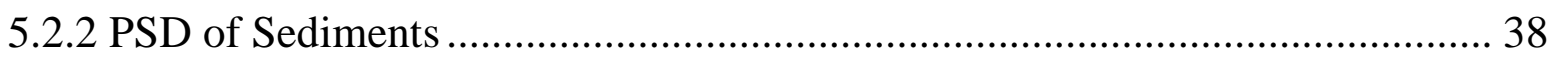

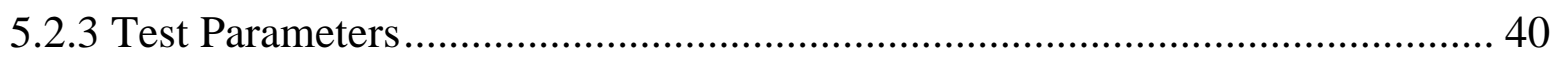

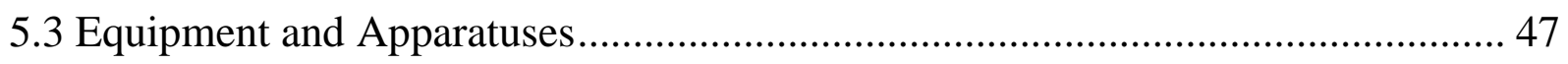

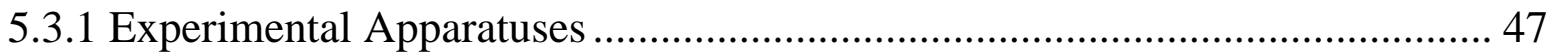

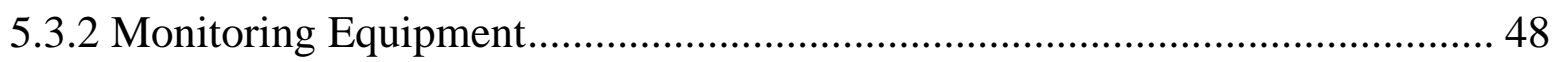

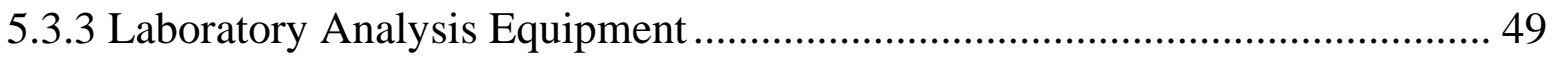

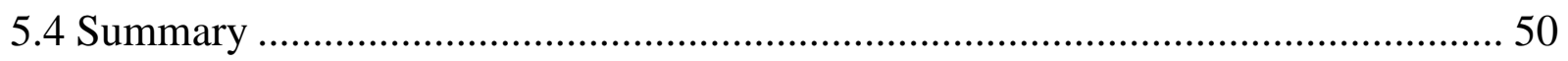




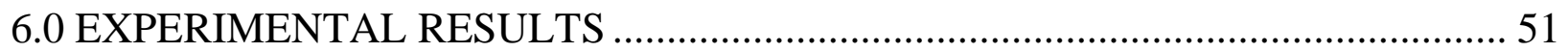

6.1 Laboratory Results and Analysis ........................................................................ 51

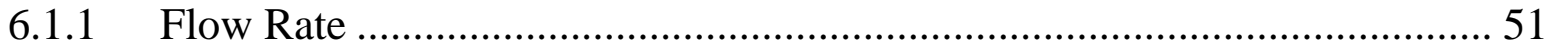

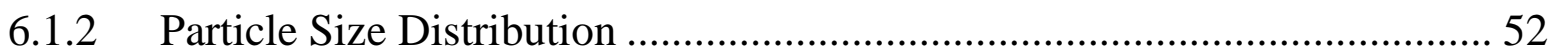

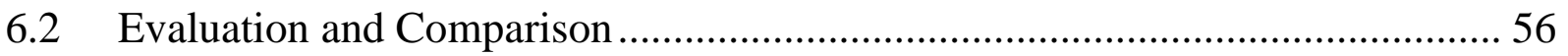

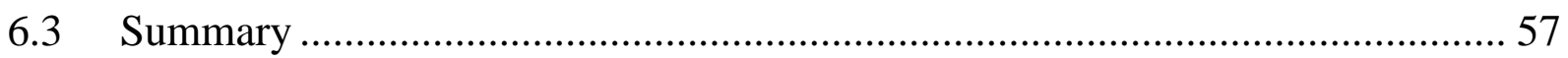

7.0 CONCLUSIONS AND RECOMMENDATIONS ................................................... 58

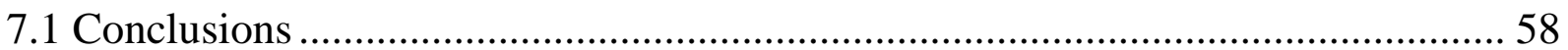

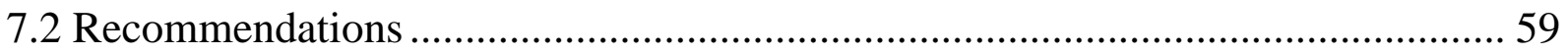

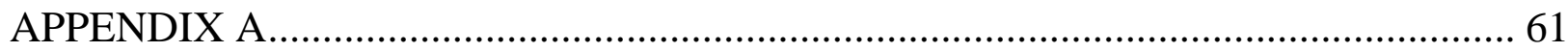

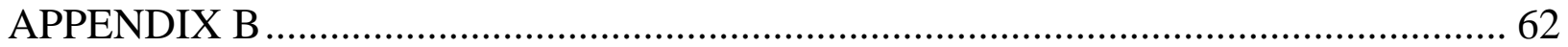

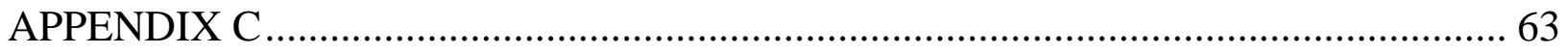

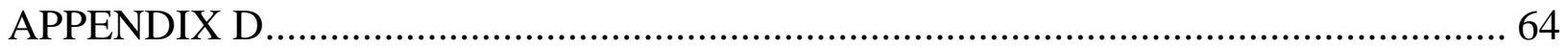

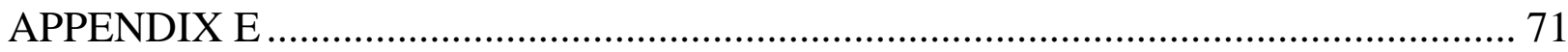

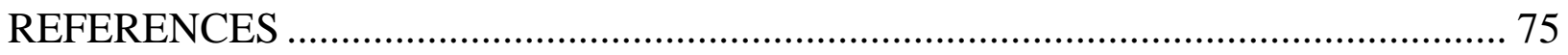




\section{List of Tables}

Table 1 Related Parameters in the Outdoor Drainage Design Standard of China ........... 24

Table 2 Road and Traffic Management Facilities Design Standards in China ................ 25

Table 3 Statistics of Event-Mean Concentrations (EMCs) for Storm Events in Beijing . 31

Table 4 Particle Size Distribution Required for the Removal Testing ............................. 40

Table 5 Modified Particle Size Distribution for Laboratory Experiments ........................ 40

Table 6 Required SLRs and the Corresponding Flow Rates.......................................... 41

Table 7 Mean, Standard Deviation and Coefficient of Variation of Two Flow Rates..... 43

Table 8 Individual Preparation for Sediments with Different Sizes ................................ 45

Table 9 Comparison between Key Requirements Stated in TRCA's Procedure and

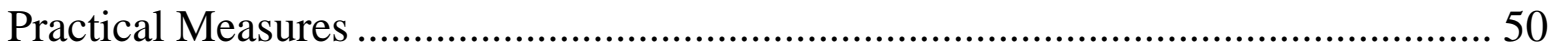

Table 10 Particle Size Distribution, Accumulation Range, Fluctuation Threshold, and

Mass of Influent Sediments ..................................................................................... 52

Table 11 Removal Efficiency Results of Effluent Sediments' Particle Size Distribution 53

Table 12 Recorded Overall and Individual Sediments Removal Results (5 L/s Test Flow) 54

Table 13 Recorded Overall and Individual Sediments Removal Results $(10$ L/s Test

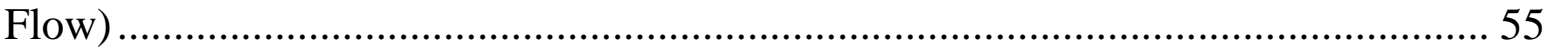




\section{List of Figures}

Figure 1 Illustration of Feeding Chamber ................................................................. 18

Figure 2 Illustration of Central Vertical Outlet Pipe and Arc Baffle ............................... 19

Figure 3 Diagram of Treatment Process of Experimental Vortex Device ……............... 20

Figure 4 Photograph of the Physical Model of Experiment Vortex Device (5 L/s)........ 20

Figure 5 Photograph of the Physical Model of Experiment Vortex Device $(48$ L/s)....... 21

Figure 6 Diagram of the Off-line System of Experimental Vortex Device along with

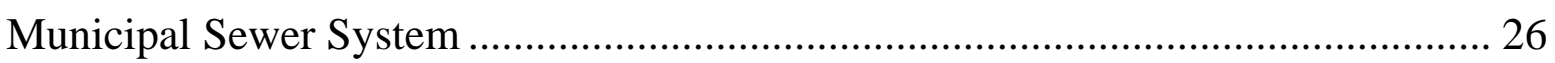

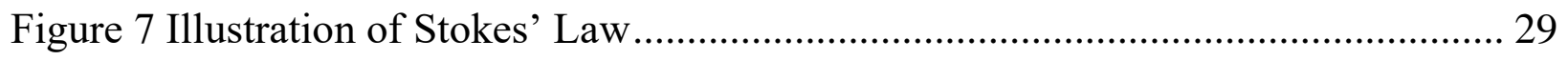

Figure 8 Proportional Comparison of Individually Sized Treatment Efficiencies between

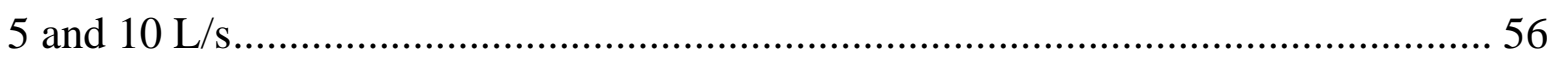




\section{Abbreviations}

(Alphabetic Order)

- $\boldsymbol{A D V}$ : Acoustic Doppler Velocimeter

- BMP: Best Management Practice

- CFD: Computational Fluid Dynamics

- COV: Coefficient of Variation

- CSO: Combined Sewer Overflows

- EVD: Experimental Vortex Device

- HDVS: Hydrodynamic Vortex Separators

- IDF Curve: Intensity-Duration-Frequency Curve

- IPS: Inclined Plate Settler

- LID: Low Impact Development

- MOE: Ministry of Environment

- MTD: Manufactured Treatment Device

- $O \& G$ : Oil and Grease

- OGS: Oil/Grit Separator

- PSD: Particle Size Distribution

- SUDS: Sustainable Urban Drainage System

- SWM: Stormwater Management

- TRCA: Toronto and Regions Conservation Authority

- TSS: Total Suspended Solids

- WSUD: Water Sensitive Urban Design

- WTP: Water Treatment Plant

- WWF : Wet Weather Flow 


\section{Terminology}

- $\lambda$ : Scale Factor, or Scale Ratio

- $L_{p}$ : Value of Characteristics of Prototype in One-Dimension

- $L_{m}$ : Value of Characteristics of Scaled Model in One-Dimension

- $R_{e}$ : Reynolds Number

- $u$ : Velocity of the Fluids

- $D$ : Diameter of the Tube

- $v$ : Kinematic Viscosity of the Fluids

- $F_{r}$ : Froude Number

- L: Hydraulic Depth

- $\log _{2}$ : Binary Logarithm (i.e., lg)

- $T$ : Return Period (year)

- $i$ : Rainfall Intensity ( $\mathrm{mm} / \mathrm{min})$

- $t / t_{c}$ : Time of Concentration (min or s)

- $A_{s u}$ : Controlled Surface Area $\left(\mathrm{m}^{2}\right)$

- $C$ : Runoff Coefficient (no unit)

- $Q_{r}$ : Surface Runoff ( $\mathrm{m}^{3} / \mathrm{s}$ or $\left.\mathrm{L} / \mathrm{s}\right)$

- $v$ : Manning's Velocity $(\mathrm{m} / \mathrm{s})$

- $n$ : Manning's n (no unit)

- $R$ : Hydraulic Radius (m)

- A: Area of Pipe $\left(\mathrm{m}^{2}\right)$

- $P$ : Wetted Perimeter (m)

- $D$ : Diameter of Pipe (m)

- $S$ : Slope of Pipe (\%)

- $v_{p}$ : Velocity in Pipe $(\mathrm{m} / \mathrm{s})$

- $Q_{p}$ : Flow Rate in Pipe $\left(\mathrm{m}^{3} / \mathrm{s}\right)$

- $Q$ : Discharge Flow Rate $\left(\mathrm{m}^{3} / \mathrm{s}\right)$ 
- $C_{d}$ : Coefficient of Discharge (no unit)

- $A_{o r}$ : Area of Orifice $\left(\mathrm{m}^{2}\right)$

- $g$ : Acceleration from Gravity $\left(\mathrm{m} / \mathrm{s}^{2}\right)$

- $h$ : Head acting on the Centerline (m)

- $F_{d}$ : Drag Force (newtons)

- $\eta$ : Viscosity of Fluid $(\mathrm{kg} / \mathrm{m} / \mathrm{s})$

- $r$ : Radius of the Particle (m)

- $u$ : Terminal Velocity of Particle $(\mathrm{m} / \mathrm{s})$

- $F_{g}$ : Gravity of the Particle (newtons)

- $\rho_{s}$ : Density of Particle $\left(\mathrm{kg} / \mathrm{m}^{3}\right)$

- $\rho_{f}$ : Density of Fluid $\left(\mathrm{kg} / \mathrm{m}^{3}\right)$

- $d$ : Diameter of Particle (m)

- $C_{s}$ : Concentration of TSS (mg/L)

- $V_{r a}$ : Volume of Stormwater Generated per Rainfall Event $\left(\mathrm{m}^{3}\right)$

- $M_{s}$ : Mass of Sediments Generated per Rainfall Event $(\mathrm{kg})$

- $V_{s}$ : Volume of Sediments Generated per Rainfall Event $\left(\mathrm{m}^{3}\right)$

- $V_{s-m a x}$ : Maximum Volume of Sediments $\left(\mathrm{m}^{3}\right.$ or $\left.\mathrm{L}\right)$

- $r_{m}$ : Radius of Manhole (m)

- $h_{s}$ : Height of Accumulated Sediments (m)

- $P$ : Liquid Pressure ('w' for 'water', 'o' for 'oil', Pa)

- $\rho_{w}$ : Density of Pure Water $\left(\mathrm{kg} / \mathrm{m}^{3}\right)$

- $\rho_{o}$ : Density of Oil $\left(\mathrm{kg} / \mathrm{m}^{3}\right)$

- $h_{w}:$ Height of Water Layer (m)

- $M_{o}$ : Mass of Oil Generated per Rainfall Event $(\mathrm{kg})$

- $C_{o}$ : Concentration of Oil (mg/L)

- $V_{o}$ : Volume of Oil Generated per Rainfall Event ( $\mathrm{m}^{3}$ or L)

- $V_{e}$ : Effective Storing Volume for Oil $\left(\mathrm{m}^{3}\right)$

- $V_{m}$ : Volume of Manhole $\left(\mathrm{m}^{3}\right)$ 
- $V_{\text {cvop: }}$ Volume of Central Vertical Outlet Pipe $\left(\mathrm{m}^{3}\right)$

- $r_{c v o p}$ : Radius of Central Vertical Outlet Pipe (m)

- $h_{o}$ : Thickness of Oil Layer (m)

- $V_{\text {chamber: }}$ Volume of the Sedimentation Chamber $\left(\mathrm{m}^{3}\right)$

- $R_{\text {device: }}$ Radius of the Device (m)

- $h_{\text {chamber }}$ : Height of the Sedimentation Chamber (m)

- $T_{\max }$ : Maximum Time for Eight Complete Volume Exchanges (min)

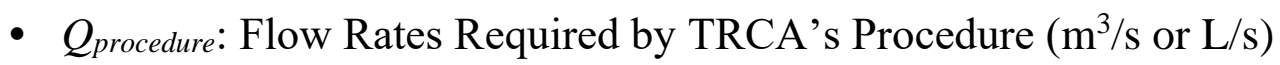

- $Q_{\text {test }}$ Test Flow Rate (L/s)

- $T_{d u}$ : Test Duration (min)

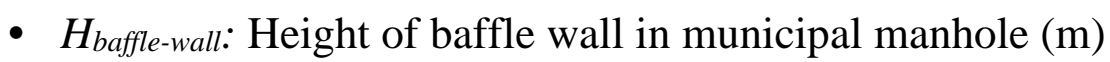

- $M_{\text {s-test: }}$ Sediments' Total Mass (g)

- $M_{s-5}$ : Sediments' Total Mass under 5 L/s Flows (g)

- $M_{s-10}$ : Sediments' Total Mass under 10 L/s Flows (g) 


\subsection{INTRODUCTION}

This chapter overviews the common stormwater problems occur in highly urbanized areas, followed by the development and challenges of the stormwater management of all time including the background information of stormwater management of the People's Republic of China. Finally, the objectives and scope of this research as well as the structure of the paper are described.

\subsection{Stormwater Problems}

Generating from unpredictable precipitation events, stormwater has been, always is and still will be bringing a series of troubles and nuisances. The two main categories of which, can be labeled as the quantity and quality issues. In recent decades, rapid urbanization has resulted in the proportional increase of total impervious areas contributed by all the buildings, pavements, roads, parking lots and other surfaces. Unfortunately, what coinciding with this change is the aggravation of the stormwater problems.

In terms of quantity issues, without enough natural adjustment strength, large amount of urban stormwater cannot be controlled by impoundment, infiltrating and evapotranspiration, as good as before, resulting in a growing number of observed urban floods. Furthermore, a greater volume, sooner and higher peak of post-development storm runoff tends to happen when compared to those of the pre-developed conditions ( $\mathrm{Li}, 2019)$. On the other hand, quality issues are also severe. The pollutions from non-point sources have brought all sorts of problems. For example, the greatest proportion among all sources of the total phosphorus loading to the Lake Simcoe, is generated by urban stormwater runoff with a value of $31 \%$ (Ontario, 2010). Large amount of uncontrolled surface runoff containing sediments, oil, nutrients, heavy metals, thermal pollutants, pathogens, 
will adversely affect the receiving water bodies and bring unwanted outcomes such as eutrophication, dissolved oxygen reduction, bad odor, water ecological imbalance and so on. Many mitigation solutions (e.g., erosion control, plant covering) have been executed in order to abate stormwater pollution (Wikipedia - Water Pollution, 2020). However, it is extremely timeconsuming and costly to achieve the stormwater management goal.

\subsection{Stormwater Management Development}

Traced back to approximately $3500 \mathrm{BC}$, with the occurrence of the first-ever human settlement, stormwater management (SWM) was emerged (Angelakis, 2017). As the civilization gradually became mature, stormwater management started to be focusing on the quantity control of stormwater (Koloski-Ostrow, 2015). Prior to 1980's, the objective of the management was primarily to convey the flows away from the living zone in a more convenient, safe and economical way. After 1990s, stormwater quality control started to emerge and has been valued by civil engineers and experts since then in Canada (Li, 2019). In 2003, the Ontario's Ministry of Environment (MOE) published the Stormwater Management Planning and Design Manual. The manual indicates that "mitigating the effects of urbanization on the hydrologic cycle" is the ultimate goal of the SWM, while an improper decision can lead to adverse consequences such as "water quality degradation, baseflow reduction, flooding and erosion, loss of properties and human lives, etc." (MOE, 2003). At present, the manual (MOE, 2003) and other related acts have been developed elaborately to provide a thorough guidance to SWM projects in Ontario by considering the control of water quantity as well as quality at the very same time. 


\subsection{Challenges}

Stormwater management has never been an easy task to fulfill with the presence of all sorts of challenges. For starters, urban areas, especially downtown areas of old large cities, are highly developed and occupied. It is very complicated to build, repair or reconstruct the infrastructures of stormwater management because of the limited space, heavy traffic and tremendous population density. Secondly, although many stormwater management (SWM) practices (e.g., low impact developments, best management practices, etc.) are widely adopted in many counties, some cannot bring out the expected benefits due to all kinds of limitations (e.g., application conditions, cost, local policy, public acceptance, operation and maintenance, etc.). The suitability of SWM practices applied at the designated place is the most important prerequisite, without which the efficiency will not be satisfying. What is more, the great difficulty of background information collection and analysis can also be the barrier of SWM. The characteristics such as surface topography, soil type, hydrology and hydrogeology, groundwater depth, climate and ecology conditions may vary greatly among different subdivisions, cities, or watersheds. With such a high variability and versatility, most of the cases cannot be compared or equalized and thus the detailed data shall be collected and organized separately for site specific stormwater management. These challenges have been the stumbling block on the road of SWM. Proper, integrated, sustainable methods are in great need for the sake of human beings and the nature.

\subsection{Backgrounds and Limitations}

China, the country which has the largest population in the world, has been developing with an extremely high rate over the recent decades. "Between 1980 and 2012, China's urbanization has increased from $19.4 \%$ to $52.6 \% "$ (Science, 2013), and there were more than 10 million people 
moving to the city from rural districts every year. Therefore, if improper stormwater management plan was adopted under this circumstance, problems such as urban flooding, water body pollution, water ecology deterioration, etc. will be induced by the drastic increase of the urban stormwater runoff (Chou, 2015).

The concept of 'Sponge City' was firstly presented by one of the deputies of Chinese National People's Congress Bo Liu in the year of 2011. The idea is to build and reconstruct cities like a sponge which can be able to absorb and hold the stormwater long enough for subsequent cyclic utilization and in the meantime, treat the initial proportion of rainwater (i.e. first flush). Similar to the terms such as Best Management Practices (BMPs) and Low Impact Developments (LID) in North America, Sustainable Urban Drainage Systems (SUDSs) in England, Water Sensitive Urban Designs (WSUDs) in the Middle East and Australia, Sponge City has shown its suitability relative to China's stormwater management programs (Cui et al., 2016). In 2015, a batch of pilot cities were selected to implement the stormwater control practices because of the high frequency of urban flooding. One year after, another batch was chosen, and there are now more than 30 cities in total which scattered all over China. After few years of experiments, some achievements have gradually showed up, and the frequency of urban flooding along with the water pollution level have been, to some extent, reduced and abated.

However, due to the short term of field-experiments and a lack of connection between theories and practices, the results were not as good as what people expected. The structure of stormwater management planning has not yet been systematized, and at the same time, the existing technology and financial status cannot support enough amount of pilot tests and advanced devices for scientific reasons. Furthermore, the understanding towards stormwater management in China was 
not comprehensive and mature enough. It is not realistic to blindly complied with other successful examples of stormwater control practices in other countries or regions (Cui et al., 2016). Instead, a grand plan which requires efforts from and cooperation among all levels of participants (i.e., ministry, municipalities, publics) should be enacted according to China's national condition.

\subsection{Objective and Scope}

The main objective of this research project is to investigate the challenges of applying the regulatory guideline titled the "Procedure for Laboratory Testing of Oil/Grit Separators" (TRCA, 2014) by conducting laboratory tests of Total Suspended Solids (TSS) removal efficiency determination of an Experimental Vortex Device (EVD).

The specific research scopes can be listed as follows:

1. Reviewing the "Procedure for Laboratory Testing of Oil/Grit Separators" (TRCA, 2014) and summarizing the essential requirements for the physical testing;

2. converting the theoretical standards to practical solutions in the real-case operations;

3. providing reliable comments on experimental procedure to benefit other related scholarly researches and to prevent them from unnecessary wasting of time or funding;

4. determining the TSS removal efficiency of the EVD and analyzing the results;

5. presenting all the acquired results and the conclusion of the analysis; and

6. comparing the treatment efficiency with other commonly-seen products. 


\subsection{Structure of the Report}

In the remaining content of this report, Chapter 2 will cover the reviews of related literatures and materials regarding the specific technology on oil/ sediments removal; Chapter 3 will present the conceptual illustration of the model of EVD along with the treating process; Chapter 4 will give the methodology of this project with applied mathematical principles and calculations; Chapter 5 will be introducing TRCA's Procedure and the experimental process in detailed way; Chapter 6 will exhibit all the testing results; and Chapter 7 will wrap up the report by giving conclusions and recommendations for the future development. 


\subsection{LITERATURE REVIEW}

The difficulty of urban stormwater management has been persistently increased since the first occurrence (Fletcher et al., 2015). There are many stormwater management technologies that are developed serving different purposes as requested. Among all the stormwater quality control and improving devices, Hydrodynamic Vortex Separator (HDVS) as well as Oil/Grit Separator (OGS), are the most frequently used technologies in the SWM industry. Derived from sufficient reviews of the literatures, reports of products associated with above-mentioned technologies, materials and news, the concepts of HDVS and OGS are reviewed accordingly. Since this research project is based on physical model investigations, the fundamental concepts of physical hydraulic modelling are overviewed.

\subsection{Review of Hydrodynamic Vortex Separators}

Hydrodynamic Vortex Separator (HDVS), or Hydrodynamic Separator, is one of the most popular methods used for the solid-liquid or liquid-liquid separation in SWM to control stormwater runoff pollution in highly urbanized areas where available space is limited. In 1960s, Bernard Smisson firstly came up with the concept of HDVS and developed it to treat the combined sewer overflows (CSOs) (Andoh, et al., 2002). Since then, many devices and products have been developed and used for both wastewater and stormwater treatment. This section reviews the applications of HDVSs and their performances.

According to Smisson (1967), the first generation of vortex separators which designed for CSOs treatment, has an overall efficiency of $70 \%$ of pollutant removal. Owing to Smisson's pioneering contribution, more produces have emerged since then. In 1970s, the next generation of HDVS - 
USEPA Swirl Concentrator and Swirl Primary Separator were developed by the American Public Works Associations and EPA with the assistance of Smisson (Sullivan, et al., 1978; Andoh \& Smission, 1994; Field \& O'Connor, 1996; Li, 2009). Development of HDVS was continued in United Kingdom. Storm King®, an improved edition of HDVS, was invented to outperformance the USEPA Swirl Concentrator by solving the demerits of which, such as generating large head loss at higher flow rates, etc. in 1980s (Andoh \& Saul, 2003). Due to the great performance and high separation efficiency, Storm King ${ }^{\circledR}$ was further developed and commercialized in UK and USA afterwards (Li, 2009).

More products inheriting the principles and advantages from the previous versions of HDVS have been developed and utilized aiming for stormwater quality treatment till nowadays, while the performances somehow vary among applications which have different properties such as operation conditions (e.g., scouring, overflows), sediments characteristics, and so forth (Li, 2009; Wikipedia - Hydrodynamic Separator, 2020). Furthermore, HDVS are found to be not as efficient when the concentration of dissolved or fine pollutants is high (USEPA, 1999; Wikipedia Hydrodynamic Separator, 2020) and according to Sullivan et al. (1972), the HDVS can be very efficient if the grain sizes are larger than or equal to 0.2 millimeter (Li, 2009).

The process of pollution treatment in HDVS can be described briefly as follows: after entering the feed chamber, the untreated stormwater will follow a downward spiral route along the chamber's sidewall and generate a centrifugal force around the rotational axis (Raiyani, 2018). In the meantime, the working principle of HDVS is rather straight forward to be explained. Generating from swirling vortex, the centrifugal force enhances the ability of the removal of Total Suspended Solids (TSS) and oil/ floatables. Furthermore, the turbulence and the velocity of the inflow can be 
reduced because of the energy consumption by the water swirls. In general, HDVS can treat pollutants having different densities from water. In other words, the heavier pollutants such as sediments, or similarly, lighter ones such as oil and floatable debris, can be separated from the water. In terms of the removal of TSS in HDVS, sediments will be centrifuged and concentrated along the peripheral wall. Those particles having larger sizes tend to settle easier and faster, while those having the smaller sizes will be affected less by the centrifugal force, and hence be more difficult to be removed (Sullivan et al., 1972). The oil and floatable debris, on the other hand, having smaller densities when comparing to the water, can be separated as well. However, instead of rotating outwards from the axis, lighter matters will concentrate centrally in these cases.

The application of HDVS in practical life is very broad. In some wastewater treatment plants, vortex-typed grit chambers can be installed before the primary treatment units for the pretreatment purposes. According to Metcalf \& Eddy, there are three different types of vortex grit chambers: “(1) Mechanically Induced Vortex; (2) Hydraulically Induced Vortex; and (3) Multitray Vortex Grit Separator" (Metcalf \& Eddy, 2014). The theory of the treatment is that: within the treatment unit, particles will travel with the water currents having the swirling flow path. By using the centrifugal force generated by swirl (i.e., vortex) and the gravitational force generated from earth, simultaneously, the grits can be separated from the water (Metcalf \& Eddy, 2014). Except for the application in the wastewater treatment plants, HDVSs also have high demand in urban areas. Products such as Aqua-Swirl ${ }^{\mathrm{TM}}$, Downstream Defender®, Dual Vortex Separator, are the most popular technologies over these years. 


\subsection{Review of Oil/Grit Separators}

Similar with the hydrodynamic vortex separators, Oil/Grit Separator (OGS), another type of SWM underground structure, is also preferred by the clients who have needs of treating solids, oil and floatables (e.g., debris, trash) contained in the stormwater runoff and/or snowmelt. Although the working processes may vary for different designs of OGS, the main principle remains the same, which is to remove TSS by sedimentation and separate oil from stormwater by phase separation. It is worth mentioning that OGS is not designed for stormwater quantity control but for quality control; nevertheless, it can somehow provide a temporary storage for stormwater and thus attenuate the peak flows (TRCA - SWAMP Program, 2004).

The scope of the applications of OGS is typically limited to small areas with large impermeability such as parking lots, gas stations, etc. Various types of OGS with different designs and functional attributes can be found in stormwater treatment industry, for example, traditional three-chamber OGS, Stormceptor®, Downstream Defender® (can also be considered as a HDVS as mentioned in previous sub-chapter), and Vortechs (product of Contech Engineered Solutions LLC.). These products are the most popular ones nowadays (TRCA - SWAMP Program, 2004).

The following literature review of OGS focuses on the designs and performances of a standard three-chamber OGS and Stormceptor® (the model of STC 4000) based on the performance assessment report prepared by SWAMP Program of TRCA in 2004. "Both technologies were installed as two parallel units in the parking lots of large Home Depot stores" and served 2.2 and 2.6 hectares of design drainage area, respectively (TRCA - SWAMP Program, 2004). 
According to the performance assessment report prepared by SWAMP Program of TRCA (2004), the precast concrete three-chamber OGS at the Home Depot store consisted of three connecting chambers: (1) The first chamber served as a grit chamber which removed the grits and heavy pollutants washed off by stormwater from the surface; (2) the second chamber (i.e., the oil chamber) was designed to trap the free oil and floatable trash or debris by setting an elbow pipe with a submerging intake in between the second and third chamber; and (3) the third chamber allowed the treated water to discharge from the OGS to the receiving sewer. The height of inlet and outlet pipe of a three-chamber OGS determines the permanent pool level inside the structure, which is approximately $1.5 \mathrm{~m}$ to $1.8 \mathrm{~m}$ deep. During wet-weather flow conditions, overflows will occur over the openings located at the top of two interior walls and exit the OGS to provide safety to the treatment system. At the Home Depot site, there were 60 runoff events monitored and a total of 26 influent as well as 54 effluent water samples collected from May 1997 to December 1998 for the evaluation of water quality improving performance of this structure. The results indicated that the median influent and effluent TSS concentration were 109 and $40 \mathrm{mg} / \mathrm{L}$, while that of oil and grease $(\mathrm{O} \& \mathrm{G})$ were 22 and $8 \mathrm{mg} / \mathrm{L}$, respectively. Additionally, the total TSS load and O\&G removal efficiency were 57\% and 51\% respectively (TRCA - SWAMP Program, 2004). On the other hand, the report stated that Stormceptor ${ }^{\circledR}$ has a different treating process. During small-flow conditions, surface runoff will follow a downward route and enter the treatment chamber (i.e., the bottom part of the structure) because of the weir and drop pipe arrangement near the inlet pipe. The grits and heavy pollutants will drop and settle down to the base while the oil and other lighter substances will rise and trapped by the outlet pipe with a submerged intake (similar to the setting of the elbow pipe of the three-chamber OGS described earlier). In comparison, during high-flow conditions, stormwater will overtop the weir and flows into the bypass chamber (i.e., the upper part of the structure), and hence be conveyed to the outlet sewer 
directly. According to the manufacturer, excessive flows will not cause re-suspension of settled pollutants as well as the trapped $O \& G$ in the treatment chamber because of the upper and lower chamber design. In terms of the water quality improving performance, a total of 20 influent and 37 effluent samples were collected from August 1997 to December 1998 at the Home Depot site. The results indicated that the median influent and effluent TSS concentration were 112 and 48 $\mathrm{mg} / \mathrm{L}$, while that of $\mathrm{O} \& \mathrm{G}$ were 17 and $7 \mathrm{mg} / \mathrm{L}$, respectively. Furthermore, the total TSS and O\&G load removal efficiency was $60 \%$ and $44 \%$ respectively). The report also compared the most common types of the OGS products, and from which a typical range of $57 \%$ to $60 \%$ of TSS removal and a range of $44 \%$ to $51 \%$ of O\&G treatment were observed (TRCA - SWAMP Program, 2004).

\subsection{Review of Other SWM Technologies}

Other than the above two technologies, there are many other designs that are available and suitable for different kinds of application conditions. For example, an enhanced treatment technology called Jellyfish ${ }^{\circledR}$ Filter designed by Imbrium also provides higher pollutant removal efficiency in many stormwater quality treatment projects. "Featuring pretreatment and membrane filtration in a compact stand-alone system, Jellyfish ${ }^{\circledR}$ Filter removes floatables, trash, oil, debris, TSS, fine silt-sized particles” and many kinds of pollutants (Imbrium Systems Inc., n.d.).

\subsection{Physical Hydraulic Modeling}

Although a full-scaled model was used in this research, it is necessary to introduce some basic concepts associated with physical hydraulic modeling. According to "Hydraulic Modeling Concepts and Practice - ASCE Manuals and Reports on Engineering Practice No. 97”, one of the 
most common physical modeling techniques - hydraulic modeling, is widely applied in hydraulicrelated engineering industry for simulating and/or evaluating the performance of scaled models replicating specific characteristics of the prototypes of hydraulic structures or machines (Ettema, 2000). In other words, a physical hydraulic model is a 'tool' used for finding the solutions to the optimal performance, both technically and economically (Heller, 2011; Novak, 1984), and can assist decision-makers during planning and design process (Chanson, 1999). An overview of related concepts and terms is presented below: Similarity; dominant forces in both free-surface and close-conduit model; some common dimensionless numbers; and the non-negligible effects.

\subsubsection{Similitude}

Similitude, i.e. similarity, "is a concept applicable to the testing of engineering models, mainly in hydraulic and aerospace engineering, to test fluid flow conditions with scaled models." (Wikipedia - Similitude (Model), 2019). If a physical model is considered to be completely similar to its prototype, the model must display similarities in terms of shape (i.e., geometric similarity), motion (i.e., kinematic similarity), and forces (i.e., dynamic similarity) (Chanson, 1999; Heller, 2011).

Among the three, geometric similarity indicates that all the characteristics of scaled models in one-dimension (e.g., length, diameter, height, etc.) shall be $\lambda$ times smaller than of its prototype, where $\lambda$ is the scale factor which can be described by Eq. (1) ('p' stands for 'prototype' while 'm' means 'model') (Heller, 2011; Chanson, 1999; Yalin, 1971; Kobus, 1980; Novak, 1984; Hughes, 1993). 


$$
\lambda=\frac{L_{p}}{L_{m}}
$$

Where,

$\lambda$ : scale factor, or scale ratio;

$L_{p}:$ value of characteristics of prototype in one-dimension;

$L_{m}$ : value of characteristics of scaled model in one-dimension.

Therefore, characteristics in two- (e.g., area) and three- (e.g., volume) dimensions will have the scale factors of $\lambda^{2}$ and $\lambda^{3}$, respectively, if the geometric similarity has been satisfied (Heller, 2011). On the other hand, kinematic similarity, requiring constant ratios of characteristics such as velocity and acceleration, implies the similarity of motions while dynamic similarity demonstrates identical ratios of force-related characteristics between the scaled model and prototype at all times.

\subsubsection{Forces}

In Fluid Dynamics, it is important to, firstly, determine the dominant forces of flow mechanism when modeling water flows in physical models. According to Hughes (1993), the most commonly-seen forces that are important to the flows are: inertial force, gravitational force, viscous force, surface tension force, etc. (Heller, 2011). Noted that two main types of flows are covered in this research: free-surface flows (also called open-channel flows) and closed conduit flows (i.e., pipe flows), and their dominant forces are different. In free-surface flows, the gravitational force will dominate (Chanson, 1999) while in closed conduit flows, the viscous force will be the one who controls the flow mechanism. Although both gravitational-based and viscousbased modeling are important, it is impossible to duplicate both gravity and viscosity in a physical 
model, in other words, to create a scaled model that satisfies both the Froude (gravity dominated) and Reynold (viscosity dominated) number. Luckily, a 1:1 scaled-model (i.e., the same size as the prototype) was developed in this research in accordance to the Procedure. If a scaled model is used, a relative basis of the Froude-based model can be used to determine whether there will be scale effect associated with the negligence of viscous forces.

\subsubsection{Dimensionless Numbers}

In Fluid Mechanics, there are many dimensionless numbers that are applied to analyze the behavior of fluids. Among all, Reynolds number $(\mathrm{Re})$ is one of the most common dimensionless parameters. Defined as the ratio of inertial force and viscous force, Reynolds number is used to predict flow patterns in different situations where viscous forces dominate and is given by Eq. (2) below.

$$
R_{e}=\frac{u D}{v}
$$

Where,

$u$ : velocity of the fluids;

$D$ : diameter of the tube;

$v$ : kinematic viscosity of the fluids.

The Froude number $\left(\mathrm{F}_{\mathrm{r}}\right)$, applied for scaling open channels or hydraulic structures (Chanson, 1999), is a ratio of inertial force and gravitational force which describes the flow regimes of freesurface flows and is given by Eq. (3). When $F_{r}$ is equal to 1, the flow is called the critical flow; when $F_{r}$ is larger than 1 , a fast and rapid supercritical flow can be observed; and when $F_{r}$ is smaller 
than 1, the flow tends to be slow and tranquil (subcritical flow). Moreover, according to Heller (2011), "Froude number similarity is especially suited for models where friction effects are negligible or for short, highly turbulent phenomena." However, in a Froude model, parameters such as gravity, Reynold number and Weber number cannot be scaled identically between the model and the real-world prototype, and thus some non-negligible scale effects will be generated (Heller, 2011).

$$
F_{r}=\frac{u}{\sqrt{g L}}
$$

Where,

$u$ : velocity of the fluids;

$g$ : gravitational acceleration;

$L:$ hydraulic depth.

In conclusion, only the dominant mechanism is needed to be modelled in most cases. In closedconduit flows, it is important to keep the Reynold number identical in both scaled model and prototype because viscous is the dominated force. However, in free-surface flows, gravity effects are more important and thus a Froude number modeling shall be used. Furthermore, when the system is controlled by inertial and surface tension forces, a similarity of Weber number must be obtained in order to acquire reliable results from scaled hydraulic model (Chanson, 1999).

\subsubsection{Effects}

There are three main effects that may lead to considerable differences of performance between the scaled model and its real-world prototype: model effect, scale effect, and measurement effect. 
All of them are originated due to incorrect operations (e.g., calculation, measurement, reproduction of features, etc.) (Heller, 2011). As a result, it is fairly tricky to find out the key concerns without neglecting important relevant factors, and at the same time, not to emphasize non-significant ones in different physical experiments.

Among the three, scale effect is the most important one in this research. Resulting in deviations between scaled model measurements and prototype observations, scale effects present because of incorrect setup of scale ratio (Heller, 2011). They are generally small, but not always negligible altogether. Some may choose to use different fluids in order to keep the Reynolds and Froude number identical in both the model and prototype and to reduce the scale effects. However, it is not a practical and economical way (Chanson, 1999).

\subsection{Summary}

Being the perfect match of serving highly developed areas because of the compact size and small occupied footprint, Hydrodynamic Vortex Separators (HDVSs) and Oil/Grit Separators (OGSs) have great potential to be explored and further developed. With similarity, to some extent, HDVS and OGS technologies are both highly preferred due to the reasonable removal efficiency of sediments (i.e., TSS) and oil (and floatable debris) between $40 \%$ to $60 \%$, while some can be higher. In this research project, the principles of both HDVS and OGS are applied to an Experimental Vortex Device (EVD) with a diameter of 1.2-meter and a height of 1.8-meter for the testing of stormwater treatment performance. More details of the testing are described in the following chapters and sections. 


\subsection{CONCEPT OF THE EVD}

\subsection{Model Illustration}

After reviewing plenty of materials associated with the technologies of Hydrodynamic Vortex Separator (HDVS) and Oil/Grit Separator (OGS) for stormwater quality control industries, the idea of an Experimental Vortex Device (EVD) design was developed in this research project. The EVD can be implemented in any municipal manhole, while in this research, the dimensions of the manhole was set to be having a typical size with a diameter of 1.2 meters and a height of 1.8 meters.

From the very beginning to the final design, the appearance and structure of EVD has been revised and improved repeatedly. The final version of EVD model has two major components: (1) Feeding chamber (which is similar to the regular municipal manholes, except for the location of the inlet and outlet pipe); and (2) the central vertical outlet pipe along with an arc baffle. The illustrations of two constituent parts are shown in Figs. 1 and 2.

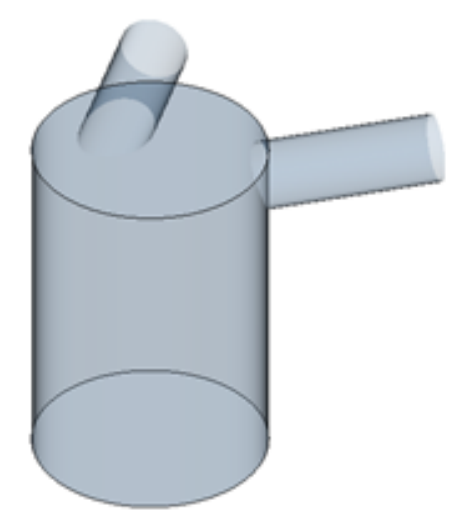

Figure 1 Illustration of Feeding Chamber 


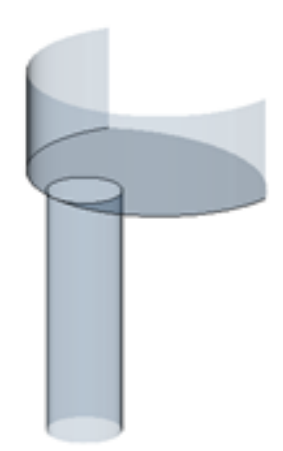

Figure 2 Illustration of Central Vertical Outlet Pipe and Arc Baffle

\subsection{Treatment Process}

Theoretically, after entering the manhole, polluted stormwater will spin along the circular sidewall and generate a centrifugal force on the fluid, which forms a vortex flow pattern. Larger suspended particles will be pulled away from the center while small ones will drop down onto the bottom and hence be settled. On the other hand, the lighter oil and floatables will float above the water. Most of them will be trapped by the arc baffle and cannot submerge into the bottom to escape from the central vertical outlet pipe, and thus be removed from the stormwater.

Figure 3 presents the treatment process of the EVD. Stormwater (brown curves) flows into the feeding chamber via inlet pipe, and then follows a downward spiral flow path. The larger particles will be removed by the centrifugal force while smaller ones will travel with the flow. A part of the smaller particles will settle down after a while, nevertheless the rest will escape the treatment system. In the meantime, lighter pollutants such as oil and debris will float above and be trapped by the arc baffle. Therefore, the treated clean water (light blue curves) will leave the device tangentially though the central vertical outlet pipe and be discharged outside the EVD. 


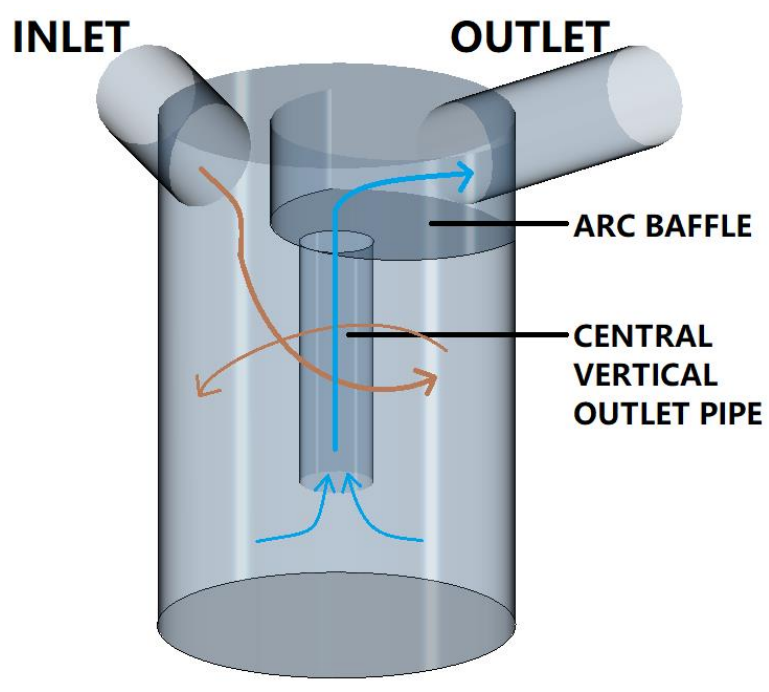

Figure 3 Diagram of Treatment Process of Experimental Vortex Device

\subsection{The Physical Model}

Figures 4 and 5 show the physical model at the hydraulic laboratory in Tianjin, China. Two flow rates representing both small and large rainfall events were tested at the physical model.

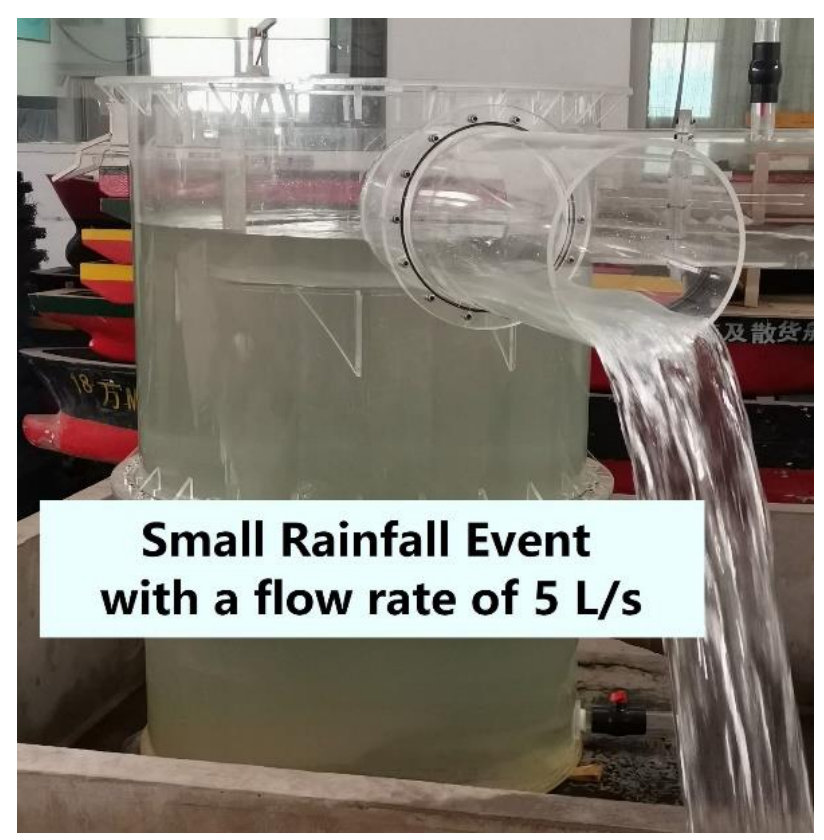

Figure 4 Photograph of the Physical Model of Experiment Vortex Device $(5 \mathrm{~L} / \mathrm{s})$ 


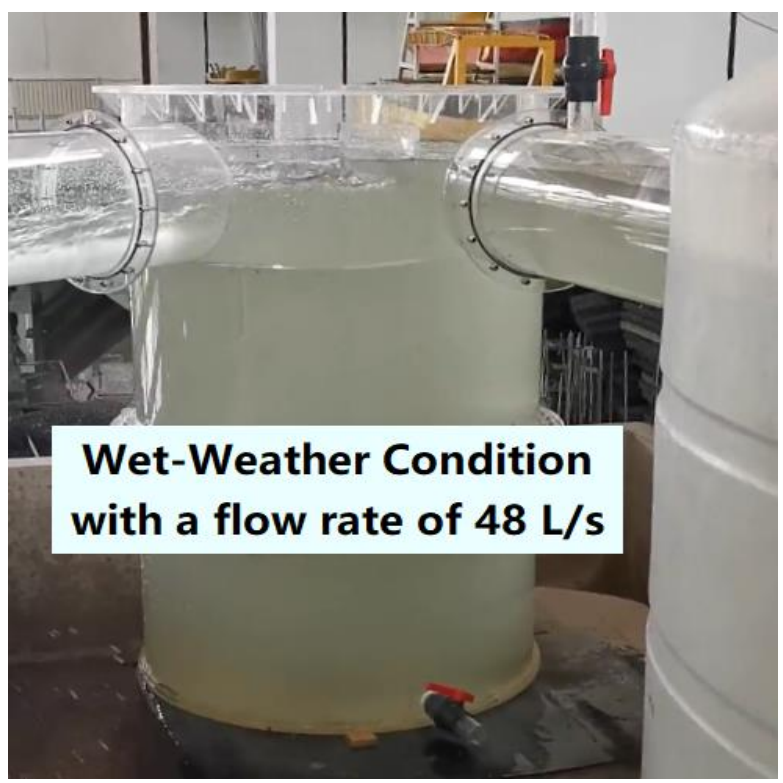

Figure 5 Photograph of the Physical Model of Experiment Vortex Device $(48 \mathrm{~L} / \mathrm{s})$

\subsection{Different Scenarios of the Setup}

Although the typical design of EVD has been determined, various feasible combinations can be applied to the model by tweaking and adjusting the variables of the model.

1. Angles between pipes: Theoretically, any angle (between the inlet and outlet pipe) that is in the range of $0^{\circ}$ to $180^{\circ}$ can be applied to the model. However, only the typical setup with an angle of $60^{\circ}$ between pipes was applied to the EVD for physical testing and experimental use because of the time and funding limits.

2. Flow rates: Flow rate is also one of the variables of the model setup. Different flow rates contribute to different sets of combinations of scenarios. Details of the testing flow range are described in the following chapter.

3. Particle size distribution (PSD): PSD is the most important variable in this research, different PSD combinations can be related to different application conditions in real cases. 


\subsection{Summary}

In this chapter, the illustration of a designed experimental vortex device (EVD) and the schematic graph showing the treating process of the device were presented. Although there are many alike products in the SWM industry, the EVD in this research, which adopts to different setups and variables, has its own features and characteristics. As a result, a thorough plan of laboratory testing with sufficient amount of hydraulic experiments is needed in order to find out the regularity and treatment efficiency of the EVD. More details regarding the model setups and the experiment results are presented in the following contents. 


\subsection{METHODOLOGY}

The fundamental calculations and assumptions related to the theories and principles applied in the development of EVD are described in this chapter. It is worth mentioning that both Beijing and the city of Tianjin, China are chosen to be the representative cities and their hydrological/ hydraulic data as well as the regulatory standards are used for the development of EVD.

\subsection{Hydrologic Characteristics}

\subsubsection{Rainfall Intensity}

According to a research about the precipitation trend analysis of Tianjin, China (Fan, 2011), the updated rainfall IDF curve formula in the city of Tianjin is given as follow.

$$
i=\frac{20.778+13.370 \cdot \log _{2} T}{(t+16.820)^{0.781}}(\mathrm{~mm} / \mathrm{min})
$$

Where,

$i$ : rainfall intensity, $\mathrm{mm} / \mathrm{min}$;

$\log _{2}$ : binary logarithm, i.e., $\lg$;

$T$ : return period, year;

$t$ : time of concentration, min.

Based on the Outdoor Drainage Design Standards in China (GB50014-2006, 2014), the average return period used for stormwater pipe design is from 0.5 to 1 year in China; the runoff coefficient for rational method $(C)$ in Tianjin is from 0.45 to 0.60 ; and the interval distance between two manholes shall not exceed 120 meters, accordingly. The required ranges and adopted values of these related parameters are listed in Table 1. 
Table 1 Related Parameters in the Outdoor Drainage Design Standard of China (GB50014-2006, 2014)

\begin{tabular}{|c|c|c|c|c|}
\hline & Return period $(\mathbf{T})$ & $\begin{array}{c}\text { Time of } \\
\text { concentration }(\mathbf{t})\end{array}$ & $\begin{array}{c}\text { Runoff coefficient for } \\
\text { rational method }(\boldsymbol{C})\end{array}$ & $\begin{array}{c}\text { Interval distance between } \\
\text { two manholes }\end{array}$ \\
\hline Given Range & $0.5-1.0$ year & $5-10$ minutes & $0.45-0.60$ & $<120$ meters \\
\hline Adopted Design Value & 1.0 year & 10 minutes & 0.60 & 100 meters \\
\hline
\end{tabular}

By assuming the return period (T) in Tianjin to be 1 year and the time of concentration (t) is 10 minutes, the rainfall intensity in the city of Tianjin should be:

$$
\text { intensity }=\frac{20.778+13.370 \cdot \log _{2} 1}{(10+16.820)^{0.781}}=1.59(\mathrm{~mm} / \mathrm{min})
$$

\subsubsection{Surface Runoff}

Based on the Chinese regulatory of Code for Design of Urban Road Engineering (CJJ37-2012, 2016), the average width of lanes should be 3.5 to $3.75 \mathrm{~m}$ in large cities, and that of sidewalks should be at least $2 \mathrm{~m}$, but less than $10 \mathrm{~m}$. It is assumed that the main roads in the city have four $3.5 \mathrm{~m}$ wide lanes and two $8 \mathrm{~m}$ wide sidewalks on both sides of road, then the total width of service area is $30 \mathrm{~m}$. By assuming the interval distance between the two manholes is 100 meters, the area controlled by one manhole $\left(A_{s u}\right)$ is given by:

$$
A_{\text {su }}=30 \times 100=3000 \mathrm{~m}^{2}
$$

The required ranges and adopted values of parameters in terms of road and traffic design in China are listed below (Table 2). Therefore, with the known rainfall intensity, service area and the runoff coefficient, the flow rate of surface runoff $\left(Q_{r}\right)$ is given by:

$$
Q_{r}=C i A_{s u}=0.60 \times 1.59 \times 3000 \times\left(10^{-3} / 60\right)=0.048 \mathrm{~m}^{3} / \mathrm{s}=48 \mathrm{~L} / \mathrm{s}
$$


Table 2 Road and Traffic Management Facilities Design Standards in China (CJJ37-2012, 2016)

\begin{tabular}{|c|c|c|c|c|}
\hline & $\begin{array}{c}\text { Average width } \\
\text { of lanes }\end{array}$ & Amount of lanes & $\begin{array}{c}\text { Average width of } \\
\text { sidewalks }\end{array}$ & Amount of sidewalks \\
\hline Given Range & $3.5-3.75$ meters & $2-8$ & $2.0-10.0$ meters & $1-2$ \\
\hline Adopted Design Value & 3.5 meters & 4 & 8.0 meters & 2 \\
\hline
\end{tabular}

\subsection{Hydraulic Characteristics}

\subsubsection{Manning's Formula}

Manning's formula, used to calculate the velocity of the open-channel gravity flow with the known roughness coefficient and slope of the pipe, is given by Eq. (8).

$$
v=\frac{1}{n} \cdot R^{\frac{2}{3}} \cdot \sqrt{S}
$$

Where,

v: manning's velocity, $\mathrm{m} / \mathrm{s}$;

$n$ : manning's n, assume 0.013 for plexiglass pipe;

$R$ : hydraulic radius, $\mathrm{m}$;

$S$ : slope of pipe, $\mathrm{S}=1 \%$.

Therefore, for $350 \mathrm{~mm}$ inlet pipe (under the full pipe flow condition), the hydraulic radius $(R)$, velocity $\left(v_{p}\right)$ and flow rate $\left(Q_{p}\right)$, respectively, are given by:

$$
\begin{gathered}
R=\frac{A}{P}=\frac{\pi D^{2}}{4 \times \pi D}=\frac{D}{4}=\frac{0.35 \mathrm{~m}}{4}=0.0875 \mathrm{~m} \\
v_{p}=\frac{1}{0.013} \times 0.0875^{\frac{2}{3}} \times \sqrt{0.01}=1.52 \mathrm{~m} / \mathrm{s} \\
Q_{p}=v_{p} \cdot A=1.52 \mathrm{~m} / \mathrm{s} \times 0.35^{2} \times \frac{\pi}{4}=0.146 \mathrm{~m}^{3} / \mathrm{s}
\end{gathered}
$$




\subsubsection{On-Site Layout and Profile}

\subsubsection{Off-line system}

The off-line system is applied to ensure the safety of the device as well as the treatment process (no overflows or backflows to the surface) when large storm events happened (Garbon, 2018). As shown in Figure 6, the inlet pipe of the treatment device collects stormwater at the bottom part of the upstream municipal manhole where a baffle wall is constructed. The purpose of the wall is to convey the flow to the EVD up to the maximum treating capacity while anything larger than the maximum capacity will overflow and flow to the downstream in the municipal system.

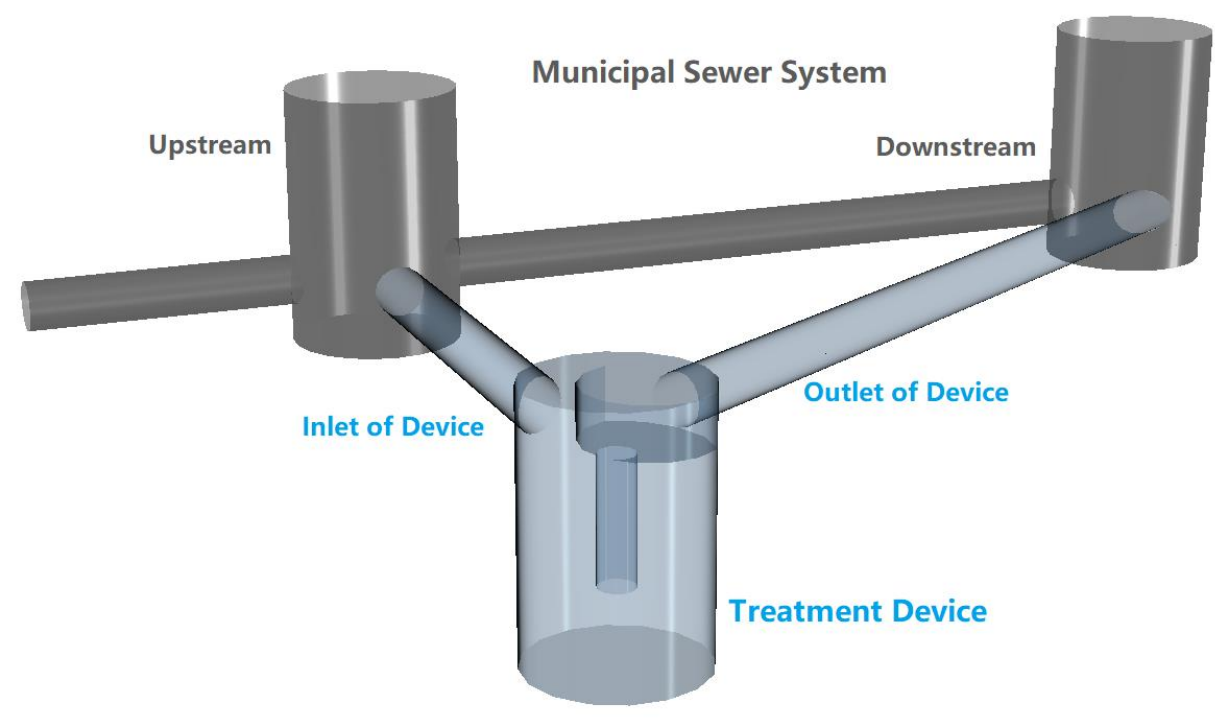

Figure 6 Diagram of the Off-line System of Experimental Vortex Device along with Municipal Sewer System (Color of Grey stands for Municipal Sewer System while Light Blue is the EVD)

\subsubsection{Orifice equation}

The orifice equation is given by Eq. (12). 


$$
Q=C_{d} A_{o r} \sqrt{2 g h}
$$

Where,

$Q$ : discharge flow rate, $\mathrm{m}^{3} / \mathrm{s}$;

$C_{d}$ : coefficient of discharge;

$A_{o r}:$ area of orifice, $\mathrm{m}^{2}$;

$g$ : acceleration from gravity, $9.81 \mathrm{~m} / \mathrm{s}^{2}$;

$h$ : head acting on the centerline, $\mathrm{m}$.

When the level of upstream stormwater reaches its highest (the upper edge of the baffle wall installed in the municipal manhole upstream), the head acting on the center of the orifice will be at the maximum value, as well as the discharge flow rate from the orifice (up to $0.146 \mathrm{~m}^{3} / \mathrm{s}$ under full-pipe flow conditions). As a result, assuming $C_{d}=0.6$ and $A=\pi r^{2}=0.096 \mathrm{~m}^{2}$, the water head above the center of the pipe, or the effective height of the baffle wall upstream, can be calculated as:

$$
h=\left(\frac{Q}{C_{d} A_{\text {or }}}\right)^{2} / 2 g=\left(\frac{0.146}{0.6 \times 0.096}\right)^{2} /(2 \times 9.81)=0.33 \mathrm{~m}
$$

Therefore, theoretically, the upstream manhole should have a baffle wall with a height of 0.805 meter to prevent overfeeding the treatment device as Eq. (14) shown.

$$
H_{\text {baffle-wall }}=0.33 m+0.175 m+0.3 m=0.805 m
$$

Where, 


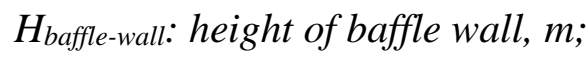

$0.33 \mathrm{~m}$ : effective height acquired in Eq. (13);

$0.175 \mathrm{~m}$ : radius of the inlet pipe;

$0.3 \mathrm{~m}$ : distance from the bottom to the lowest point of inlet pipe.

\subsection{Sedimentation}

\subsubsection{Particle Size Distribution}

According to the "Procedure for Laboratory Testing of Oil-Grit Separators Consider" (TRCA, 2014), six particle sizes were selected for sediment removal performance testing, which are 75 microns, 100 microns, 150 microns, 250 microns, 500 microns, and 710 microns, respectively. Details will be presented in the following contents.

\subsubsection{Stokes' Law}

According to the Stokes' Law, the fluid flow is assumed to be in laminar condition without turbulence, and the particles are further assumed to be spherical without interacting with each other. As shown in Figure 7, the movement or status of any particle having a diameter of ' $d$ ' (in meter) is governed by the drag force $F_{d}$ and the gravitational force $F_{g}$ (the buoyant force has been considered in the gravity term). By the time the drag force is equal to the gravity force, a balance will be achieved and there will be no acceleration exist, hence the particle will reach its terminal velocity (Tyson Ochsner, 2013). 


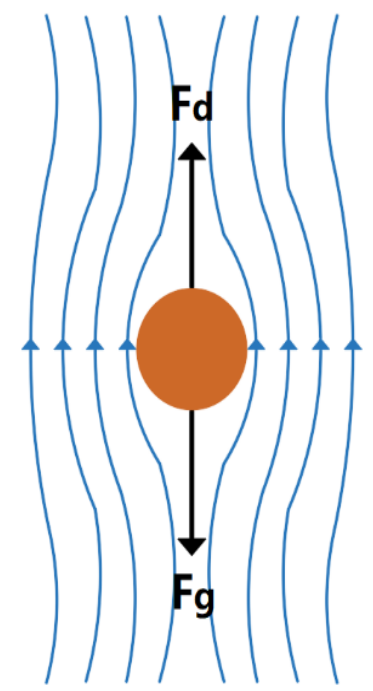

Figure 7 Illustration of Stokes' Law

(Figure Reference: https://en.wikipedia.org/wiki/File:Stokes_sphere.svg)

According to Tyson Ochsner (2013), the Stokes' Law is given by Eq. (15).

$$
F_{d}=6 \pi \times \eta \times r \times u
$$

Where,

$F_{d}:$ drag force;

$\eta$ : viscosity of fluid (water), $0.001 \mathrm{~kg} / \mathrm{m} / \mathrm{s}$ at $20^{\circ} \mathrm{C}$;

$r$ : radius of the particle, $\mathrm{m}$;

$u$ : terminal velocity, $\mathrm{m} / \mathrm{s}$.

Secondly, the formula of gravitational force incorporating the buoyant force is given by Eq. (16) (Tyson Ochsner, 2013).

$$
F_{g}=\frac{4}{3} \pi r^{3} \times\left(\rho_{s}-\rho_{f}\right) g
$$


Where,

$F_{g}$ gravity of the particle;

$\rho_{s}:$ density of particle, $\mathrm{kg} / \mathrm{m}^{3}$;

$\rho_{f}$ : density of fluid (water), $1000 \mathrm{~kg} / \mathrm{m}^{3}$;

$g$ : acceleration from gravity, $9.81 \mathrm{~m} / \mathrm{s}^{2}$.

\subsubsection{Terminal Velocity}

By equalizing $F_{d}$ and $F_{g}$, combining Eq. 15 and 16 can derive the following formula:

$$
u=\frac{d^{2} g}{18 \eta} \times\left(\rho_{s}-\rho_{f}\right)
$$

Therefore, the terminal settling velocity for particles with a diameter of $75 \mu \mathrm{m}\left(d_{50}\right)$ in $20^{\circ} \mathrm{C}$ water is given by:

$$
u_{75}=\frac{\left(75 \times 10^{-6} \mathrm{~m}\right)^{2} \times 9.81 \mathrm{~m} / \mathrm{s}^{2} \times\left(2650 \mathrm{~kg} / \mathrm{m}^{3}-1000 \mathrm{~kg} / \mathrm{m}^{3}\right)}{18 \times 0.001 \mathrm{~kg} / \mathrm{m} / \mathrm{s}}=0.005 \mathrm{~m} / \mathrm{s}
$$

Similarly, when substitute the particle sizes: $100 \mu \mathrm{m}\left(d_{60}\right), 150 \mu \mathrm{m}\left(d_{75}\right), 250 \mu \mathrm{m}\left(d_{90}\right), 500 \mu \mathrm{m}$ $\left(d_{95}\right)$, and $710 \mu \mathrm{m}\left(d_{100}\right)$ into Eq. (17), the terminal settling velocities for particles in $20^{\circ} \mathrm{C}$ water will be, respectively:

$$
\begin{aligned}
& u_{100}=\frac{\left(100 \times 10^{-6} \mathrm{~m}\right)^{2} \times 9.81 \mathrm{~m} / \mathrm{s}^{2} \times\left(2650 \mathrm{~kg} / \mathrm{m}^{3}-1000 \mathrm{~kg} / \mathrm{m}^{3}\right)}{18 \times 0.001 \mathrm{~kg} / \mathrm{m} / \mathrm{s}}=0.01 \mathrm{~m} / \mathrm{s} \\
& u_{150}=\frac{\left(150 \times 10^{-6} \mathrm{~m}\right)^{2} \times 9.81 \mathrm{~m} / \mathrm{s}^{2} \times\left(2650 \mathrm{~kg} / \mathrm{m}^{3}-1000 \mathrm{~kg} / \mathrm{m}^{3}\right)}{18 \times 0.001 \mathrm{~kg} / \mathrm{m} / \mathrm{s}}=0.02 \mathrm{~m} / \mathrm{s} \\
& u_{250}=\frac{\left(250 \times 10^{-6} \mathrm{~m}\right)^{2} \times 9.81 \mathrm{~m} / \mathrm{s}^{2} \times\left(2650 \mathrm{~kg} / \mathrm{m}^{3}-1000 \mathrm{~kg} / \mathrm{m}^{3}\right)}{18 \times 0.001 \mathrm{~kg} / \mathrm{m} / \mathrm{s}}=0.06 \mathrm{~m} / \mathrm{s}
\end{aligned}
$$




$$
\begin{aligned}
& u_{500}=\frac{\left(500 \times 10^{-6} \mathrm{~m}\right)^{2} \times 9.81 \mathrm{~m} / \mathrm{s}^{2} \times\left(2650 \mathrm{~kg} / \mathrm{m}^{3}-1000 \mathrm{~kg} / \mathrm{m}^{3}\right)}{18 \times 0.001 \mathrm{~kg} / \mathrm{m} / \mathrm{s}}=0.22 \mathrm{~m} / \mathrm{s} \\
& u_{710}=\frac{\left(710 \times 10^{-6} \mathrm{~m}\right)^{2} \times 9.81 \mathrm{~m} / \mathrm{s}^{2} \times\left(2650 \mathrm{~kg} / \mathrm{m}^{3}-1000 \mathrm{~kg} / \mathrm{m}^{3}\right)}{18 \times 0.001 \mathrm{~kg} / \mathrm{m} / \mathrm{s}}=0.45 \mathrm{~m} / \mathrm{s}
\end{aligned}
$$

\subsection{Sediments Maintenance Frequency}

\subsubsection{Event-Mean Concentrations}

According to a case study done by Shen et al. in 2016, the event-mean concentrations (EMCs) of total suspended solids captured on three different impervious surfaces in Beijing are shown in Table 3. The mean value of TSS concentration at main traffic roads is equal to $373.77 \mathrm{mg} / \mathrm{L}$ (approximately $375 \mathrm{mg} / \mathrm{L}$ ).

Table 3 Statistics of Event-Mean Concentrations (EMCs) for Storm Events in Beijing ( $m g / L)$ (Shen et al., 2016)

\begin{tabular}{|l|l|c|c|c|cc|c|}
\hline Impervious Surface & Statistics & COD & TSS & TP & Fe & Zn \\
\hline \hline \multirow{4}{*}{ Roof } & Mean & 346.92 & 43.07 & 0.11 & 0.22 & 4.40 \\
& Standard Deviation & 241.88 & 31.78 & 0.11 & 0.36 & 7.52 \\
& Max & 856.67 & 99.50 & 0.42 & 1.24 & 26.49 \\
& Min & 18.95 & 14.77 & 0.01 & 0.01 & 0.21 \\
\hline \multirow{3}{*}{ Road in Residential Area } & Mean & 561.75 & 286.94 & 1.23 & 0.09 & 0.11 \\
& Standard Deviation & 719.30 & 187.96 & 1.73 & 0.08 & 0.05 \\
\hline \multirow{3}{*}{ Main Traffic Road } & Max & 2537.50 & 654.82 & 6.25 & 0.28 & 0.19 \\
& Min & 46.82 & 101.55 & 0.10 & 0.02 & 0.02 \\
\hline
\end{tabular}

However, the standard of "Procedure for Laboratory Testing of Oil-Grit Separators" (TRCA, 2014) requires that a constant concentration of $200 \mathrm{mg} / \mathrm{L}$ ( $25 \%$ of deviation) be conducted over the whole duration of each experiment (25 minutes). Therefore, at the first stage of physical experiment, the concentration of $200 \mathrm{mg} / \mathrm{L}$ is adopted prior to Beijing's EMC because of the time 
limit and the alignment with Canadian regulation. The next stage of TSS removal experiment will focus on a higher concentration of $375 \mathrm{mg} / \mathrm{L}$ according to Shen et al. (2016).

\subsubsection{Sediments Clearance}

\subsubsection{Hydrograph}

It is assumed that the hydrograph of the place of interest (i.e., Beijing or the city of Tianjin, in $\mathrm{m}^{3} / \mathrm{s}$ ) has a triangular shape with a base of $2.67 t_{c}$ and a peak of $Q_{r}(\mathrm{Li}, 2018)$. The area of this triangle is the volume of the stormwater per designed rainfall event, which can be determined as follows (the letters 'ra' stand for 'rainfall' while ' $\mathrm{t}_{\mathrm{c}}$ ' is 'time of concentration'):

$$
\begin{gathered}
2.67 t_{c}=2.67 \times 10 \min =26.7 \min =1602 \mathrm{~s} \\
V_{r a}=0.5 \times 2.67 t_{c} \times Q_{r}=0.5 \times 1602 \mathrm{~s} \times 0.048 \mathrm{~m}^{3} / \mathrm{s}=38.45 \mathrm{~m}^{3}=38450 \mathrm{~L}
\end{gathered}
$$

The volume of sediments generated per rainfall event can thus be determined by Eq. (26).

$$
V_{s}=\frac{M_{s}}{\rho_{s}}=\frac{V_{r a} \times C_{s}}{\rho_{s}}=\frac{\left(38450 \mathrm{~L} \times 200 \mathrm{mg} / \mathrm{L} \times 10^{-6}\right) \mathrm{kg}}{2.650 \mathrm{~kg} / \mathrm{L}}=2.90 \mathrm{~L}
$$

Where,

$M_{s}$ : mass of sediments generated per rainfall event;

$V_{r a}$ : volume of stormwater generated per designed rainfall event, computed as $38.45 \mathrm{~m}^{3}$;

$C_{s}:$ concentration of TSS;

$V_{s}$ : volume of sediments generated per rainfall event;

$\rho_{\mathrm{s}}$ : density of sediments, $2.650 \mathrm{~kg} / \mathrm{L}$. 
Since the device has a deposit bottom with a height of 0.5 meter, the sediments accumulation should not exceed the lower edge of the central vertical outlet pipe. The maximum capacity of the bottom of EVD (i.e., total volume of accumulated sediments) is given by the following equation.

$$
V_{s-\max }=\pi \times\left(r_{m}\right)^{2} \times h_{s}=0.565 m^{3}=565 \mathrm{~L}
$$

Where,

$V_{s-m a x}:$ maximum total volume of sediments;

$r_{m}$ : radius of manhole;

$h_{s}$ : height of accumulated sediments.

\subsubsection{Clearance Frequency}

The total number of designed rainfall events $(\mathrm{T}=1$ year $)$ can be determined as follow:

$$
\frac{V_{s-\max }}{V_{S}}=\frac{565 \mathrm{~L}}{2.90 \mathrm{~L}}=195
$$

Therefore, theoretically, the sediments will be accumulated fully at the deposit bottom of EVD after about 195 times of 1-year rainfall event, and hence maintenance is needed. However, the rainfall events are always stochastic and unpredictable, and the practical clearance frequency shall be determined based on actual observation and analysis.

\subsection{Oil Removal}

Supposedly, after rain stops, the water level inside the device will gradually decline and then stay unchanged when it reaches the lower edge of outlet pipe. Since the arc baffle is blocking the floating substances from escaping the treatment device, the oil and floatable debris will be trapped 
behind the arc baffle and be stayed afloat above the water layer. All these floating substances will generate a hydraulic head which pushes down the oil-water interface and squeeze out a bit of water to the downstream through the outlet pipe.

Based on the liquid pressure formula $P=\rho g h$, the pressure of both water and oil can be determined. It is worth mentioning that the density of pure water $\left(\rho_{w}\right)$ is $1000 \mathrm{~kg} / \mathrm{m}^{3}$, which is considered as the density of the stormwater; meanwhile, the density of petroleum is approximately $800 \mathrm{~kg} / \mathrm{m}^{3}$, which is considered as the density of oil $\left(\rho_{o}\right)$ contained in the surface runoff.

$$
\begin{gathered}
P_{w}=\rho_{w} h_{w} \cdot g \\
P_{o}=\rho_{o} h_{o} \cdot g
\end{gathered}
$$

Where,

$P$ : liquid pressure ('w' for 'water', 'o' for 'oil', Pa);

$\rho$ : density of liquid ('w' for 'water', 'o' for 'oil', $\mathrm{kg} / \mathrm{m}^{3}$ );

$h$ : height or thickness of liquid layer, ('w' for 'water', 'o' for 'oil', m);

$g$ : acceleration from gravity, $9.81 \mathrm{~m} / \mathrm{s}^{2}$.

By equalizing two liquid pressures, the relationships between the thickness of the oil and the height of the water at the steady state is given by:

$$
1000 \cdot h_{w}=800 \cdot h_{o}
$$

Therefore,

$$
h_{w}=0.8 \cdot h_{o}
$$


There are two critical cases at which oil will escape from the treatment system: (1) When overflow occurs from the arc baffle; and (2) when the oil-water interface reaches the lower edge of the central vertical outlet pipe.

The first case is relatively harder to control, because the water level will change with the inflow rate. When the inflow rate is large, the water level will rise, hence not much of the oil can be kept inside the device behind the arc baffle, let alone the critical situation when the water level exceeds the arc baffle and generate overflows. However, for the second case, some assumptions can be made based on the equations above. When the height of water layer is $500 \mathrm{~mm}$ (the height of the sediments deposit bottom), the theoretical thickness of oil layer is $625 \mathrm{~mm}$ which is the maximum distance from the lowest edge of the central vertical outlet pipe to the top of the oil layer. By that time, oil cleaning-up is needed in order to prevent any leakage.

The average concentration of oil pollutants $\left(C_{o}\right)$ in initial period of surface runoff in Beijing is 65 $\mathrm{mg} / \mathrm{L}$, and the coefficient of variation varies from 0.4 to 2.0 (Che et al, 2003). Therefore, by assuming the coefficient of variation to be 2.0, Beijing's maximum concentration of oil pollutants can be computed to be $130 \mathrm{mg} / \mathrm{L}$. Therefore, the mass as well as the volume of oil generated per designed rainfall event can be determined respectively by Eq. 33 and 34 .

$$
\begin{gathered}
M_{o}=V_{r a} \times C_{o}=38450 \mathrm{~L} \times 130 \mathrm{mg} / \mathrm{L} \times 10^{-6}=5.0 \mathrm{~kg} \\
V_{o}=\frac{M_{o}}{\rho_{o}}=\frac{5.0 \mathrm{~kg}}{0.8 \mathrm{~kg} / \mathrm{L}}=6.25 \mathrm{~L}
\end{gathered}
$$

Where, 
$M_{o}$ : mass of oil generated per rainfall event, $\mathrm{kg}$;

$V_{r a}:$ volume of stormwater generated per designed rainfall event, computed as $38.45 \mathrm{~m}^{3}$;

$C_{o}$ : concentration of oil (i.e., petroleum), $\mathrm{kg} / \mathrm{L}$;

$V_{o}$ : volume of oil generated per rainfall event, $\mathrm{L}$;

$P_{o}$ : density of oil (i.e., petroleum), $0.8 \mathrm{~kg} / \mathrm{L}$.

The difference between the volume of the larger cylinder (i.e., manhole) and that of the smaller cylinder (i.e., central vertical outlet pipe) is the effective storing volume for accumulated oil with a maximum thickness of $0.625 \mathrm{~m}$ and is given by Eq. 35 below.

$$
V_{e}=V_{m}-V_{c v o p}=\pi \times\left[\left(r_{m}\right)^{2}-\pi\left(r_{p}\right)^{2}\right] \times h_{o}=0.676 m^{3}=676 L
$$

Where,

$V_{e}$ : effective storing volume for oil;

$V_{m}$ : volume of manhole;

$V_{c v o p}$ : volume of central vertical outlet pipe;

$r_{m}$ : radius of manhole;

$r_{c v o p}$ : radius of central vertical outlet pipe;

$h_{o}$ : thickness of oil layer.

\subsection{Summary}

In this chapter, all the associated mathematical calculations as well as the theoretical principles were introduced where the basic characteristics related to the hydrology, hydraulics, sedimentation, and maintenance frequency, were determined step by step. 


\subsection{EXPERIMENTAL PROCEDURE}

The main purpose of this research is to investigate the challenges of using the regulatory guideline, prepared by TRCA (2014), which titled "Procedure for Laboratory Testing of Oil/ Grit Separators" (referred to in this paper as the "Procedure"), to test TSS/ water separation performance of device. By conducting the laboratory experiments on an innovative EVD with similar designs combining typical features of HDVS and OGS technologies, detailed conclusions and recommendations can be developed for future users of the Procedure.

\subsection{Standards and Protocols}

The setup of the physical model and the associated laboratory experiment process followed the Procedure (TRCA, 2014) primarily. Other than this, the criterion developed by the New Jersey Corporation for Advanced Technology (NJCAT) titled "Procedure for Obtaining Verification of a Stormwater Manufactured Treatment Device from New Jersey Corporation for Advanced Technology: for use in accordance with the Stormwater Management Rules, N.J.A.C. 7:8” (2013) is also a guideline for this research. NJCAT's procedure clearly established the rules for obtaining the certificate from the NJDEP (New Jersey Department of Environmental Protection) by those manufacturers who have developed the manufactured stormwater treatment devices and would like to be verified by NJCAT. Furthermore, associated regulations and local by-laws regarding the assembling of model and usage of experimental analyzing facilities, etc. in Beijing and/or the city of Tianjin, China, are the important standards of this research as well. For instance, as mentioned in Section 4.1.1 and 4.1.2, the rainfall IDF curve formula in the city of Tianjin (Fan, 2011); the requirements regarding the average return period, the runoff coefficient $(C)$, and the 
interval distance between two manholes (GB50014-2006, 2014); along with the road and sidewalk design standards in China (CJJ37-2012, 2016), have been specified earlier in this report.

\subsection{Procedural Details}

In this section, the details of Procedure are described because they are the key requirements to follow in order to conduct a successful physical test with a high reliability and validity. Some adjustments of the Procedure were needed to ensure the testing can be conducted within the limitations of the laboratory and material supplies.

\subsubsection{Scale Requirements}

According to the TRCA procedure, "the tested manufactured treatment device (MTD) must be a full scale" with identical configuration and components to the greatest extent for the practical insitu installation. Obviously, a 1:1-real-scale experimental vortex device (EVD) model will ensure the authenticity and the reliability of the results because no scaling and transforming of results will be involved, which also reduce the difficulty of the numerical analysis using the acquired data.

\subsubsection{PSD of Sediments}

In order to estimate the mass of sediments generated per rainfall event and then successfully conduct laboratory experiments, the Particle Size Distribution (PSD) of surface runoff at the place of interest is mandatorily required. 
According to a research ( $\mathrm{Li}$ et al., 2003) with more than 10 storm events during the year from 2001 to 2002, the PSD from different surfaces of urban areas in Beijing were obtained. Li et al. conducted the sedimentation experiments based on the PSD and the results showed that the equivalent scattered light particle diameters D50 and D97 were within $50 \mu \mathrm{m}$ and $100 \mu \mathrm{m}$ respectively; equivalent settling velocity diameters D50 and D90 were within $25 \mu \mathrm{m}$ and $90 \mu \mathrm{m}$ respectively. It is concluded that the equivalent scattered light particle diameter and the equivalent settling velocity diameter be used to represent the rainwater particle sizes ( $\mathrm{Li}$ et al., 2003). However, those data were collected almost 20 years ago, urbanization has caused significant changes to urban areas in China, especially in its capital city. Therefore, a more suitable PSD data for the current status is needed.

According to the Procedure, "the test sediment used for sediment removal performance testing shall be comprised of inorganic ground silica with a specific gravity of 2.65 (i.e. $\rho_{\mathrm{s}}=2650 \mathrm{~kg} / \mathrm{m}^{3}$ ), uniformly mixed to meet the particle size distribution shown in table below (Table 4), which includes a broad range of particles from clay to coarse sand" (TRCA, 2014). However, due to the limited time of experiments and the purchasing difficulty, only six particle sizes are selected within the range for sediment removal performance testing, which are 75 microns, 100 microns, 150 microns, 250 microns, 500 microns, and 710 microns, respectively, and the particle size range will be modified to: <75 $\mu \mathrm{m}$ (50\%), 75-100 $\mu \mathrm{m}$ (10\%), 100-150 $\mu \mathrm{m}$ (15\%), 150-250 $\mu \mathrm{m}(15 \%)$, 250-500 $\mu \mathrm{m}(5 \%)$, and 500-1000 $\mu \mathrm{m}$ (5\%), accordingly (Table 5).

Furthermore, before running the tests, the sediments must be uniformly mixed. Three samples shall be taken and the average of which can vary from the 'Percent Less Than' value by $6 \%$ (e.g., for 
100-micron particles' sample, the 'Percent Less Than' value should be $60 \% \pm 6 \%$ ). If the specified value exceeds this allowance threshold, a report must be accomplished (TRCA, 2014).

Table 4 Particle Size Distribution Required for the Removal Testing (TRCA, 2014)

\begin{tabular}{|c|c|c|c|}
\hline Particle Size $(\boldsymbol{\mu m})$ & $\begin{array}{c}\text { Percentage } \\
\text { Less Than }\end{array}$ & $\begin{array}{c}\text { Particle Size } \\
\text { Fraction }(\boldsymbol{\mu m})\end{array}$ & Percentage (\%) \\
\hline \hline 1000 & 100 & $500-1000$ & 5 \\
500 & 95 & $250-500$ & 5 \\
250 & 90 & $150-250$ & 15 \\
150 & 75 & $100-150$ & 15 \\
100 & 60 & $75-100$ & 10 \\
75 & 50 & $50-75$ & 5 \\
50 & 45 & $20-50$ & 10 \\
20 & 35 & $8-20$ & 15 \\
8 & 20 & $5-8$ & 10 \\
5 & 10 & $2-5$ & 5 \\
2 & 5 & $<2$ & 5 \\
\hline
\end{tabular}

Table 5 Modified Particle Size Distribution for Laboratory Experiments

\begin{tabular}{|c|c|c|c|}
\hline Particle Size $(\boldsymbol{\mu m})$ & $\begin{array}{c}\text { Percentage } \\
\text { Less Than }\end{array}$ & $\begin{array}{c}\text { Particle Size } \\
\text { Fraction }(\boldsymbol{\mu m})\end{array}$ & Percentage (\%) \\
\hline \hline 710 & 100 & $500-1000$ & 5 \\
500 & 95 & $250-500$ & 5 \\
250 & 90 & $150-250$ & 15 \\
150 & 75 & $100-150$ & 15 \\
100 & 60 & $75-100$ & 10 \\
75 & 50 & $<75$ & 50 \\
\hline
\end{tabular}

\subsubsection{Test Parameters}

\subsubsection{Background conditions}

There are two requirements need to be satisfied for the conditions of background. Firstly, five background TSS water samples shall be taken from the water source, and the average concentration of which must not exceed $20 \mathrm{mg} / \mathrm{L}$ in order to make sure accumulative effects from the system will not be generated. Secondly, the water temperature shall be lower than $25^{\circ} \mathrm{C}$ (TRCA, 2014). 


\subsubsection{Flow rates and duration}

The tested flow rates were chosen to be align with requirements and empirical cases in Beijing and the city of Tianjin, China. Due to the time limitations, finance and technical conditions, only $5 \mathrm{~L} / \mathrm{s}$ and $10 \mathrm{~L} / \mathrm{s}$ were selected to be tested. Nonetheless, there are seven surface loading rates (SLRs, or Surface Overflow Rates) that are required by the Procedure. Full range of flow rates shall be tested for future research. Moreover, all the hydraulic experiments shall be conducted under the steady-state condition.

Table 6 Required SLRs and the Corresponding Flow Rates (TRCA, 2014)

\begin{tabular}{|c|c|}
\hline Required SLRs $\left(\mathrm{L} / \mathrm{min} / \mathrm{m}^{2}\right)$ & Flow Rates $(\mathrm{L} / \mathrm{s})$ \\
\hline \hline 40 & 0.75 \\
80 & 1.51 \\
200 & 3.77 \\
400 & 7.54 \\
600 & 11.31 \\
1000 & 18.85 \\
1400 & 26.39 \\
\hline
\end{tabular}

In addition to 5 and $10 \mathrm{~L} / \mathrm{s}$, more flow rates shall be tested in the future experiments as required by the Procedure. As Table 6 shown above, the required SLRs: "40, 80, 200, 400, 600, 1000 and $1400 \mathrm{~L} /$ min per square meter of the effective treatment area" (TRCA, 2014) are given in the left column (effective treatment area is the sedimentation area of the device). As a result, the corresponding flow rates can be determined (given in the right column of Table 6). An example of transforming the required SLR to the corresponding flow rate is given by:

$$
\text { Flow Rate }(\mathrm{L} / \mathrm{s})=40 \mathrm{~L} / \mathrm{min} / \mathrm{m}^{2} \times \pi \times(0.6 \mathrm{~m})^{2}=45.24 \mathrm{~L} / \mathrm{min}=0.75 \mathrm{~L} / \mathrm{s}
$$


The Procedure states that the test duration shall be either 25 minutes or "the time for 8 complete volume exchanges in primary sedimentation chamber", whichever is greater (TRCA, 2014). Since the model has a sedimentation chamber with a dimension of 0.5 -meter in depth and 0.6 -meter in radius, the volume as well as the time for 8 complete volume exchanges under $5 \mathrm{~L} / \mathrm{s}$ (critical situation) is given by the following equations.

$$
\begin{gathered}
V_{\text {Chamber }}=\pi \times R_{\text {device }}{ }^{2} \times h_{\text {chamber }}=\pi \times 0.6^{2} \times 0.5=0.565 \mathrm{~m}^{3}=565 \mathrm{~L} \\
T_{\max }=\frac{8 \times V_{\text {Chamber }}}{Q_{\text {procedure }}}=\frac{8 \times 565 \mathrm{~L}}{5 \times 60(\mathrm{~L} / \mathrm{min})}=15 \mathrm{~min}
\end{gathered}
$$

Where,

$V_{\text {chamber: }}$ volume of the sedimentation chamber;

$R_{\text {device: }}$ radius of the device;

$h_{\text {chamber: }}$ height of the sedimentation chamber;

$T_{\text {max }}$ : maximum time for 8 complete volume exchanges;

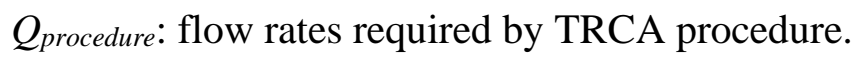

Therefore, in this research, all the tests were conducted over the same duration of 25 minutes after reaching the steady state flows. However, the duration of tests might be different when flow rates required by the Procedure are applied. For the cases of SLR equals 40 and $80 \mathrm{~L} / \mathrm{min} / \mathrm{m}^{2}$, the time required for 8 volume exchanges will be a lot larger than 25 minutes. In those cases, the duration shall be adjusted accordingly. In addition, the flow rates should be recorded at a maximum 30second interval throughout the whole experiment period and shall not exceed the fluctuation threshold of $\pm 10 \%$ with a Coefficient of Variation (COV) less than 0.04 . 
As shown in Table 7, the flow measurements retrieved from three batches of experiments, which were collected every 30 seconds over 25 minutes for both 5 and $10 \mathrm{~L} / \mathrm{s}$ flow, are analysed. The COV requirement was met perfectly for all records, and the value of the average flows fell within the $\pm 10 \%$ threshold as well (e.g., for $5 \mathrm{~L} / \mathrm{s}$, the range is $4.5 \mathrm{~L} / \mathrm{s}$ to $5.5 \mathrm{~L} / \mathrm{s}$ ). Additionally, the flow measurement results also demonstrated the steadiness of the inflow rates and the high reliability of the flow condition.

Table 7 Mean, Standard Deviation and Coefficient of Variation of Two Flow Rates

\begin{tabular}{|c|c|c|c|}
\hline \multirow{2}{*}{ Flow Rate: $5 \mathrm{~L} / \mathrm{s}$} & \multicolumn{3}{|c|}{ Batch No. } \\
\hline & 1 & 2 & 3 \\
\hline Mean & 4.92 & 5.07 & 5.01 \\
\hline Standard Deviation & 0.12 & 0.17 & 0.08 \\
\hline $\mathrm{COV}$ & 0.02 & 0.03 & 0.02 \\
\hline \multirow{2}{*}{ Flow Rate: $10 \mathrm{~L} / \mathrm{s}$} & \multicolumn{3}{|c|}{ Batch No. } \\
\hline & 1 & 2 & 3 \\
\hline Mean & 9.89 & 9.75 & 10.05 \\
\hline Standard Deviation & 0.20 & 0.21 & 0.25 \\
\hline $\mathrm{COV}$ & 0.02 & 0.02 & 0.03 \\
\hline \multicolumn{4}{|c|}{ Note: $5.0 \mathrm{~L} / \mathrm{s} \pm 10 \% \rightarrow 4.5 \mathrm{~L} / \mathrm{s} \sim 5.5 \mathrm{~L} / \mathrm{s} ; 10.0 \mathrm{~L} / \mathrm{s} \pm 10 \% \rightarrow 9.0 \mathrm{~L} / \mathrm{s} \sim 11.0 \mathrm{~L} / \mathrm{s}$} \\
\hline
\end{tabular}

\subsubsection{Influent TSS concentration and the injection location}

According to the Procedure, a sediment feeding instrument should be calibrated before use. The requirement of a constant delivery of TSS concentration of $200 \mathrm{mg} / \mathrm{L}$ with $\pm 25 \mathrm{mg} / \mathrm{L}$ fluctuation throughout the whole test duration of 25 minutes must be satisfied by the feeding instrument. In addition, the distance between the injection point of the sediments and the inlet of device shall be either 3 meters or 5 times as much as the diameter of the inlet pipe, whichever's smaller (TRCA, 2014). Since the diameter of the inlet pipe of EVD is 0.35 meter, so the sediment auto-feeding 
machine was installed at the point of 1.75 -meter $(5$ times $0.35 \mathrm{~m})$ upstream of the device's inlet. Once the flow rate becomes steady, a uniform sediment injection shall be immediately initiated.

\subsubsection{Preparation of Sediments}

Since the influent flow should have a constant concentration of $200 \mathrm{mg} / \mathrm{L}$ throughout the whole duration of 25 minutes (TRCA, 2014), with the fixed test flow rates and modified PSD (presented in Table 5), the total mass of sediments needed for each experiment can be determined by Eq. (39). Table 8 presents the masses needed for sediments with different sizes.

$$
M_{s-t e s t}=Q_{\text {test }} \times T_{d u} \times C_{s}
$$

Where,

$M_{\text {s-test: }}$ sediments' total mass, $\mathrm{g}\left(\mathrm{M}_{\mathrm{s}-5}\right.$ and $\mathrm{M}_{\mathrm{s}-10}$ stand for total mass under $5 \mathrm{~L} / \mathrm{s}$ and $10 \mathrm{~L} / \mathrm{s}$ flows);

$Q_{\text {test: }}$ test flow rate, $5 \mathrm{~L} / \mathrm{s}$ or $10 \mathrm{~L} / \mathrm{s}$;

$T_{d u}$ : test duration, 25 minutes;

$C_{s}$ : concentration of sediments, $0.2 \mathrm{~g} / \mathrm{L}$.

Therefore, the corresponding total mass required per test under 5 and $10 \mathrm{~L} / \mathrm{s}$ will be:

$$
\begin{gathered}
M_{s-5}=5 L / s \times(25 \times 60) s \times 0.2 g / L=1500 g \\
M_{s-10}=10 L / s \times(25 \times 60) s \times 0.2 g / L=3000 g
\end{gathered}
$$


Table 8 Individual Preparation for Sediments with Different Sizes

\begin{tabular}{|c|c|c|c|}
\hline Particle Size $(\boldsymbol{\mu m})$ & Percentage (\%) & Mass(gram, $\mathbf{5} \mathbf{~ L / s )}$ & Mass(gram, 10 L/s) \\
\hline \hline 710 & 5 & 75 & 150 \\
500 & 5 & 75 & 150 \\
250 & 15 & 225 & 450 \\
150 & 15 & 225 & 450 \\
100 & 10 & 150 & 300 \\
75 & 50 & 750 & 1500 \\
\hline Total & $\mathbf{1 0 0}$ & $\mathbf{1 5 0 0}$ & $\mathbf{3 0 0 0}$ \\
\hline
\end{tabular}

\subsubsection{Collection of sediments}

At the end of each experiment, the water level will be maintained at the level of the lower edge of the outlet pipe. After 1-hr of sedimentation, the remaining water can be roughly distinguished as two zones: (1) clear water zone; and (2) deposit zone. In this research, because the test flow rates are relatively small, the influent sediments are not very significant. As a result, a practical decision was made to set the height of deposition zone to be $30 \%$ of the height of the bottom of EVD below the central outflow pipe (i.e. $500 \mathrm{~mm}$ ). The very last $150 \mathrm{~mm}$ of the remaining fluid in the device shall be collected in a proper way to prevent inaccuracy of the final results $(30 \% \times 500 \mathrm{~mm}$ $=150 \mathrm{~mm}$ ). The percentage of $30 \%$ should be adjusted (i.e., increased) when the test flow rates are larger than $10 \mathrm{~L} / \mathrm{s}$.

The water in the clear zone contains limited amount of TSS, thus can be discarded by a small suction pump. What is unique is the collecting mechanism of the trapped sediments in the deposition zone. In this zone, it is assumed that the mixture of the water and sediments is the amount of sediments trapped by the EVD. If a pump is applied to suck out this amount of water, there is a possibility that some sediments may be trapped inside the machine or elsewhere, and hence decreases the accuracy and reliability of the test results. In order to facilitate the collection 
of sediments from the bottom of EVD, a cone-shaped sloping base (5\% slope was adopted) with a 'sludge' pipe installed at the middle of the base was installed. The trapped sediment collection process can be described as follows (the brief description of the laboratory schedule can be found in Appendix A).

1. Discard the supernatant (i.e., clean water at the upper layer) by a smaller suction pump;

2. Stop pumping the water when the level approaches the deposit zone;

3. Prepare a screen with a mesh size as fine as $75 \mu \mathrm{m}$ (i.e., anything larger than or equal to this size will be trapped) and place it at the outlet of the 'sludge' pipe at the base of the model;

4. Open the valve of 'sludge' pipe to release residual liquid (mixture of water and sediments);

5. After drained out, flush the EVD model with clean water (hose might be needed);

6. Stop flushing when there are no sediments left everywhere in the model;

7. Close the valve, and remove the screen;

8. After drying, measure the mass of the collected sediments, analyse the PSD of the collected sediments, determine the individual and overall removal efficiency of TSS.

Instead of collecting sediments from the outlet pipe and calculating the difference between the mass of sediments in the influent and effluent, collection of trapped sediments at the bottom can directly show the total quantity of TSS removed by the EVD. Given the influent sediment quantity, the treatment efficiency of TSS can be determined. Furthermore, it will be very troublesome and inconvenient to collect the effluent at the outlet pipe of EVD throughout the whole 25 minutes duration, more efforts will be needed with less efficiency. 


\subsubsection{Effluent screening and analysis}

For the physical model experiments, a flow-recirculation system was adopted. In order to avoid the accumulative impact from background sediments/ oil concentration, a filter with a mesh size of $75 \mu \mathrm{m}$ (like the screen discussed earlier) was installed before the inlet of the recirculation pump. As discussed in 5.2.3.5, the sediments captured by the screen will be dried before weighted, and afterwards, be evenly mixed and analysed for the particle size distribution as per the Procedure.

\subsection{Equipment and Apparatuses}

This section specifies the essential equipment and apparatuses for the physical experiments of the TSS and/or oil removal.

\subsubsection{Experimental Apparatuses}

The necessary apparatuses for the experiments are listed below:

1. Recirculation pump: A submerged pump was selected to serve the full range of flow rate requirement. The submerged pump was Item No. QY200-10-7.5A, with specifications listed as: (1) Maximum flow rate of $200 \mathrm{~m}^{3} / \mathrm{h}$; (2) maximum hydraulic head of 10 meters; (3) power of 7.5 kilowatt; (4) rotational speed of $3000 \mathrm{r} / \mathrm{min}$; and (5) pipe inner diameter of 200 millimeters.

2. Suction pump: Clearance of clean water stored in the model after each test;

3. Weighting and measuring device: To weight and measure the mass of sediments having different particle sizes according to the percentages in Table 5;

4. Stirring machine: For uniform mixing of the sediments in accordance to the specified PSD before introducing to the inflows, as per the Procedure; 
5. Feeding instrument: Evenly distribution of all the well-prepared sediments to the inflows throughout the whole duration (i.e., 25 minutes) of each test;

6. Filter screens: Two sets of screens were used. One screen is installed at the wall in between the turbid pond and clean-water pond (see Section 7.2 for details), and another one is used to collect the captured sediments by the model at the outlet of 'sludge pipe'. The filter screens shall have a mesh size the same with the finest particle size required in the tests;

7. Drying stove: Used for drying the collected sediments. After which, mass measuring and PSD analysis of the TSS can be proceeded.

\subsubsection{Monitoring Equipment}

There were three types of monitoring instrument applied in the physical experiments, the function of which are listed below:

1. An Acoustic Doppler Velocimeter (ADV) was used to continuously monitor and record the real-time velocity vectors (in $\mathrm{X}, \mathrm{Y}$, and $\mathrm{Z}$ axis) at the designated points inside the EVD throughout the 25-min duration of tests.

2. Water level probes were used to continuously monitor and record the real-time water levels under different flow rates at three different locations simultaneously throughout the test duration of $25 \mathrm{~min}$.

3. A flow meter was used to continuously monitor the real-time flow rates and velocities of inlet pipe.

The measurements of 2 sets of velocity vectors and 3 sets of water levels inside the EVD of each experiment were used for a Computational Fluid Dynamic (CFD) model calibration. As for the 
flow meter, as discussed in 5.2.3.2, it is required by the Procedure to record the flow rates at a maximum 30-second interval throughout the whole experimental period. The flow rates will be accepted only when the (recirculation) pump can be able to provide a stable flow with a $\pm 10 \%$ fluctuation threshold and a COV that is smaller than 0.04 .

\subsubsection{Laboratory Analysis Equipment}

The equipment for laboratory analysis is one of the most indispensable 'hardware' in the research project. In this project, two types of laboratory analysis equipment are listed below:

1. PSD analyser: The analysis of the particle size distribution of both the influent and the captured TSS is the main method to determine the sediments treatment efficiency of the experimental vortex device (EVD). As described earlier, the sediments shall be well-mixed and uniformly stirred before they are introduced to the model. In order to ensure the uniformity of the sediments, as per the Procedure, "3 samples of the well mixed test sediment shall be collected and analyzed for PSD. In addition, 1 sample of the test sediment used for each flow rate test shall be collected and analyzed for PSD” (TRCA, 2014). Furthermore, the PSD of the captured sediments shall be analysed as well (after drying). By comparing the difference between the influent and the captured TSS, the performance of the overall treatment efficiency as well as the individually sized removal efficiencies can be determined accordingly.

2. Sampling bottles and the Imhoff cone: In order to measure the background TSS concentration, which cannot exceed $20 \mathrm{mg} / \mathrm{L}$ as per the Procedure, multiple (three or more) sampling points shall be adopted for a reliable result. 


\subsection{Summary}

This chapter presents the detailed experimental calculations which are required to meet the standard protocol stated in the "Procedure for Laboratory Testing of Oil/ Grit Separators" (TRCA, 2014) and the final experimental procedure based on practical considerations and laboratory limitations. Furthermore, the essential equipment and apparatuses of the experiments are specified. Table 9 summarizes the key requirements stated in the Procedure and the final laboratory scheme adopted.

Table 9 Comparison between Key Requirements Stated in TRCA's Procedure and Practical Measures

\begin{tabular}{|c|c|c|}
\hline No. & Key Requirements (TRCA, 2014) & Adopted Scheme \\
\hline $\mathbf{1}$ & Scale: $1: 1$. & Real-size physical model (D: 1.2m; H: 1.8m). \\
\hline 2 & Flow Rates: from 5 to $48 \mathrm{~L} / \mathrm{s}( \pm 10 \%$; $\mathrm{COV}<0.04)$ & $\begin{array}{l}\text { Two flow rates ( } 5 \text { and } 10 \mathrm{~L} / \mathrm{s} \text { ) were tested, both satisfied } \\
\text { the } \pm 10 \% \text { threshold with an average COV of } 0.02 \text {. }\end{array}$ \\
\hline 3 & Constant Concentration: $200 \mathrm{mg} / \mathrm{L}( \pm 25 \mathrm{mg} / \mathrm{L})$. & As required. \\
\hline 4 & $\begin{array}{l}\text { Test Duration: "Either } 25 \text { minutes or the time required } \\
\text { for eight complete volume exchanges during primary } \\
\text { sedimentation, whichever is larger." }\end{array}$ & 25 minutes. \\
\hline 5 & $\begin{array}{l}\text { PSD: "The test sediment used for sediment removal tese } \\
\text { shall be comprised of inorganic ground silica with a } \\
\text { specific gravity of } 2.65 \text {, uniformly mixed to meet the } \\
\text { required PSD (Table 4), which stands for a broad range of } \\
\text { particles from clay to coarse sand." }\end{array}$ & $\begin{array}{l}\text { Six sizes were chosen }(75 \mu \mathrm{m}, 100 \mu \mathrm{m}, 150 \mu \mathrm{m}, 250 \mu \mathrm{m} \text {, } \\
500 \mu \mathrm{m} \text {, and } 1000 \mu \mathrm{m}) \text {, as shown in Table } 5 \text {. }\end{array}$ \\
\hline
\end{tabular}




\subsection{EXPERIMENTAL RESULTS}

After conducting a series of experiments on the real-scale physical model (dimensions are given in Appendix B) under different scenarios, many sets of data were collected. For both the flow rates of $5 \mathrm{~L} / \mathrm{s}$ and $10 \mathrm{~L} / \mathrm{s}$, the obtained data include:

1. 6 sets of particle size distribution (PSD) data for influent sediments analysis;

2. 6 batches of trapped sediment samples needed for PSD analysis;

3. 3 flow rate records for the determination of the mean value and standard deviation;

4. real-time water velocity measurements at 2 locations inside the model;

5. continuous collection of the water levels at 3 different locations simultaneously.

The first to the third sets of data were used to estimate the overall and particle sized TSS treatment efficiencies while the fourth and the fifth sets of data will be used to calibrate a CFD model for future research. In this report, the discussion of experimental data focuses on the first to the third sets of data.

\subsection{Laboratory Results and Analysis}

\subsubsection{Flow Rate}

The flow rate results recorded from three batches of experiments are shown in Table 7. The COV requirement was met perfectly for all records, and the value of the average flows all fall within the $\pm 10 \%$ threshold as well. 


\subsubsection{Particle Size Distribution}

One of the main objectives of this research is to find out the treatment efficiency of experimental vortex device (EVD). As discussed earlier in Section 5.2.2, the required PSD of test sediments are listed in Table 4 (TRCA, 2014). However, due to the time limitation, modifications have been made to the required PSD (given in Table 5). The PSD, accumulation range, fluctuation threshold, and mass of influent sediments are presented in Table 10.

Table 10 Particle Size Distribution, Accumulation Range, Fluctuation Threshold, and Mass of Influent Sediments

\begin{tabular}{|c|c|c|c|c|c|}
\hline \multicolumn{6}{|c|}{ Flow Rate: $5 \mathrm{~L} / \mathrm{s}$} \\
\hline Range of Size $(\mu \mathrm{m})$ & Particle Size ( $\mu m)$ & PSD (\%) & $\begin{array}{c}\text { Accumulation } \\
\text { Range (\%) }\end{array}$ & $\begin{array}{c}\text { Fluctuation } \\
( \pm 6 \%)\end{array}$ & $\operatorname{Mass}(g)$ \\
\hline $500-1000$ & 7110 & 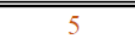 & 100 & 94-100 & 75 \\
\hline $250-500$ & 500 & 5 & 95 & $89-100$ & 75 \\
\hline $150-250$ & 250 & 15 & 90 & $84-96$ & 225 \\
\hline $100-150$ & 150 & 15 & 75 & $69-81$ & 225 \\
\hline $75-100$ & 100 & 10 & 60 & $54-66$ & 150 \\
\hline- & 75 & 50 & 50 & $44-56$ & 750 \\
\hline Sum & - & 100 & - & - & 10 \\
\hline
\end{tabular}

\begin{tabular}{|c|c|c|c|c|c|}
\hline \multicolumn{6}{|c|}{ Flow Rate: $10 \mathrm{~L} / \mathrm{s}$} \\
\hline Range of Size $(\mu \mathrm{m})$ & Particle Size $(\mu \mathrm{m})$ & PSD (\%) & $\begin{array}{c}\text { Accumulation } \\
\text { Range (\%) }\end{array}$ & $\begin{array}{c}\text { Fluctuation } \\
( \pm 6 \%)\end{array}$ & $\operatorname{Mass}(g)$ \\
\hline $500-1000$ & 710 & 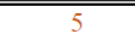 & 100 & "94-100 & 150 \\
\hline $250-500$ & 500 & 5 & 95 & $89-100$ & 150 \\
\hline $150-250$ & 250 & 15 & 90 & $84-96$ & 450 \\
\hline $100-150$ & 150 & 15 & 75 & $69-81$ & 450 \\
\hline $75-100$ & 100 & 10 & 60 & $54-66$ & 300 \\
\hline- & 75 & 50 & 50 & $44-56$ & 1500 \\
\hline Sum & - & 100 & - & - & 3000 \\
\hline
\end{tabular}

In order to satisfy the Procedure, the PSD of three samples of the well-mixed sediments for each test should have a maximum 6\% fluctuation from the average PSD. Since each test had a batch of the influent and captured sediments, six samples of sediments were collected for the PSD analysis for the two test flows. Together with the measured total mass (after drying) of each batch, the 
average overall and particle sized removal efficiencies were determined as shown in Table 11. Table 12 and 13 present the overall and particle sized sediment removal efficiencies of each sample for 5 and $10 \mathrm{~L} / \mathrm{s}$ of test flows. It is noted that the removal efficiency of $710 \mu \mathrm{m}$ in Table 12 is smaller than that of $500 \mu \mathrm{m}$ for Batch 5-3. The reason might be the instrumental errors.

Appendix C compares the overall and particle sized treatment efficiencies of the 2 test flows while Fig. 8 simplifies the bar graphs by showing the proportional comparison of particle sized treatment efficiencies between 5 and $10 \mathrm{~L} / \mathrm{s}$. In addition, line charts presenting the trend of treatment efficiency of individual sized particles as well as the trend of removal performance of all scenarios, are given in Appendix D and E respectively.

Table 11 Removal Efficiency Results of Effluent Sediments' Particle Size Distribution

\begin{tabular}{|c|c|c|c|c|c|}
\hline \multirow{8}{*}{ Flow Rate: $5 \mathrm{~L} / \mathrm{s}$} & Size $(\mu m)$ & $5-1$ & $5-2$ & $5-3$ & Average \\
\hline & 710 & $100.0 \%$ & $100.0 \%$ & $61.2 \%$ & $87.1 \%$ \\
\hline & 500 & $100.0 \%$ & $100.0 \%$ & $100.0 \%$ & $100.0 \%$ \\
\hline & 250 & $86.0 \%$ & $83.0 \%$ & $73.7 \%$ & $80.9 \%$ \\
\hline & 150 & $91.4 \%$ & $83.6 \%$ & $78.9 \%$ & $84.6 \%$ \\
\hline & 100 & $76.7 \%$ & $71.0 \%$ & $71.0 \%$ & $72.9 \%$ \\
\hline & 75 & $20.1 \%$ & $19.7 \%$ & $23.1 \%$ & $21.0 \%$ \\
\hline & Overall & $55.0 \%$ & $52.1 \%$ & $49.6 \%$ & $52.2 \%$ \\
\hline \multirow{8}{*}{ Flow Rate: $10 \mathrm{~L} / \mathrm{s}$} & Size $(\mu m)$ & $10-1$ & $10-2$ & $10-3$ & Average \\
\hline & 710 & $100.0 \%$ & $100.0 \%$ & $100.0 \%$ & $100.0 \%$ \\
\hline & 500 & $100.0 \%$ & $100.0 \%$ & $100.0 \%$ & $100.0 \%$ \\
\hline & 250 & $95.1 \%$ & $93.6 \%$ & $81.8 \%$ & $90.2 \%$ \\
\hline & 150 & $74.0 \%$ & $77.1 \%$ & $58.7 \%$ & $69.9 \%$ \\
\hline & 100 & $41.4 \%$ & $47.0 \%$ & $43.3 \%$ & $43.9 \%$ \\
\hline & 75 & $9.9 \%$ & $11.8 \%$ & $10.5 \%$ & $10.7 \%$ \\
\hline & Overall & $45.3 \%$ & $46.8 \%$ & $41.2 \%$ & $44.4 \%$ \\
\hline
\end{tabular}


Table 12 Recorded Overall and Individual Sediments Removal Results (5 L/s Test Flow)

\begin{tabular}{|c|c|c|c|}
\hline \multicolumn{4}{|c|}{ TSS Removal Record - Adjusted PSD } \\
\hline \multicolumn{4}{|c|}{ Date: 2020-Jan-10 } \\
\hline Flow $(\mathrm{L} / \mathrm{s})$ & 5 & \multirow{2}{*}{\multicolumn{2}{|c|}{$\begin{array}{c}\text { Grain Size/Sizes (microns): } 710(\mathrm{~d} 100), 500(\mathrm{~d} 95), 250 \\
(\mathrm{~d} 90), 150(\mathrm{~d} 75), 100(\mathrm{~d} 60), 75(\mathrm{~d} 50) .\end{array}$}} \\
\hline Angle of Pipes $\left({ }^{\circ}\right)$ & 60 & & \\
\hline \multicolumn{4}{|c|}{ Overall Results } \\
\hline & $\begin{array}{c}\text { Total Mass of TSS Inflow } \\
(\mathrm{kg})\end{array}$ & $\begin{array}{l}\text { Total Mass being Trapped } \\
\qquad(\mathrm{kg})\end{array}$ & Total Removal Percentage (\%) \\
\hline Batch \#1 & \multirow{3}{*}{1.5} & 0.825 & $55.0 \%$ \\
\hline Batch \#2 & & 0.782 & $52.1 \%$ \\
\hline Batch \#3 & & 0.744 & $49.6 \%$ \\
\hline Mean & - & 0.784 & $52.2 \%$ \\
\hline Standard Deviation & - & 0.041 & 0.027 \\
\hline \multicolumn{4}{|c|}{ Discrete Results } \\
\hline Size: 710 microns & Mass of TSS Inflow (kg) & Mass being Trapped (kg) & $\begin{array}{l}\text { Total Removal Percentage after } \\
\text { Correction }(\%)\end{array}$ \\
\hline Batch \#1 & \multirow{3}{*}{0.075} & 0.0408 & $100.0 \%$ \\
\hline Batch \#2 & & 0.0265 & $100.0 \%$ \\
\hline Batch \#3 & & 0.0095 & $61.2 \%$ \\
\hline Mean & - & 0.0256 & $87.1 \%$ \\
\hline Standard Deviation & - & 0.0156 & 0.224 \\
\hline Size: $\mathbf{5 0 0}$ microns & Mass of TSS Inflow $(\mathrm{kg})$ & Mass being Trapped $(\mathrm{kg})$ & $\begin{array}{c}\text { Total Removal Percentage after } \\
\text { Correction(\%) }\end{array}$ \\
\hline Batch \#1 & \multirow{3}{*}{0.075} & 0.1216 & $100.0 \%$ \\
\hline Batch \#2 & & 0.1319 & $100.0 \%$ \\
\hline Batch \#3 & & 0.1114 & $100.0 \%$ \\
\hline Mean & - & 0.1216 & $100.0 \%$ \\
\hline Standard Deviation & - & 0.0103 & 0.000 \\
\hline Size: 250 microns & Mass of TSS Inflow $(\mathrm{kg})$ & Mass being Trapped (kg) & $\begin{array}{c}\text { Total Removal Percentage after } \\
\text { Correction(\%) }\end{array}$ \\
\hline Batch \#1 & \multirow{3}{*}{0.225} & 0.1811 & $86.0 \%$ \\
\hline Batch \#2 & & 0.1782 & $83.0 \%$ \\
\hline Batch \#3 & & 0.1657 & $73.7 \%$ \\
\hline Mean & - & 0.1750 & $80.9 \%$ \\
\hline Standard Deviation & - & 0.0082 & 0.064 \\
\hline Size: 150 microns & Mass of TSS Inflow $(\mathrm{kg})$ & Mass being Trapped $(\mathrm{kg})$ & $\begin{array}{l}\text { Total Removal Percentage after } \\
\text { Correction(\%) }\end{array}$ \\
\hline Batch \#1 & \multirow{3}{*}{0.225} & 0.2056 & $91.4 \%$ \\
\hline Batch \#2 & & 0.1881 & $83.6 \%$ \\
\hline Batch \#3 & & 0.1776 & $78.9 \%$ \\
\hline Mean & - & 0.1904 & $84.6 \%$ \\
\hline Standard Deviation & - & 0.0141 & 0.063 \\
\hline Size: 100 microns & Mass of TSS Inflow $(\mathrm{kg})$ & Mass being Trapped $(\mathrm{kg})$ & $\begin{array}{c}\text { Total Removal Percentage after } \\
\text { Correction(\%) }\end{array}$ \\
\hline Batch \#1 & \multirow{3}{*}{0.150} & 0.1150 & $76.7 \%$ \\
\hline Batch \#2 & & 0.1064 & $71.0 \%$ \\
\hline Batch \#3 & & 0.1065 & $71.0 \%$ \\
\hline Mean & - & 0.1093 & $72.9 \%$ \\
\hline Standard Deviation & - & 0.0049 & 0.033 \\
\hline Size: 75 microns & Mass of TSS Inflow $(\mathrm{kg})$ & Mass being Trapped $(\mathrm{kg})$ & $\begin{array}{c}\text { Total Removal Percentage after } \\
\text { Correction }(\%)\end{array}$ \\
\hline Batch \#1 & \multirow{3}{*}{0.750} & 0.1510 & $20.1 \%$ \\
\hline Batch \#2 & & 0.1480 & $19.7 \%$ \\
\hline Batch \#3 & & 0.1732 & $23.1 \%$ \\
\hline Mean & - & 0.1574 & $21.0 \%$ \\
\hline Standard Deviation & - & 0.0138 & 0.018 \\
\hline
\end{tabular}


Table 13 Recorded Overall and Individual Sediments Removal Results (10 L/s Test Flow)

\begin{tabular}{|c|c|c|c|}
\hline \multicolumn{4}{|c|}{ TSS Removal Record - Adjusted PSD } \\
\hline \multicolumn{4}{|c|}{ Date: 2020-Jan-05 } \\
\hline Flow $(\mathrm{L} / \mathrm{s})$ & 10 & \multirow{2}{*}{\multicolumn{2}{|c|}{$\begin{array}{c}\text { Grain Size/Sizes (microns): } 710(\mathrm{~d} 100), 500(\mathrm{~d} 95), 250 \\
(\mathrm{~d} 90), 150(\mathrm{~d} 75), 100(\mathrm{~d} 60), 75(\mathrm{~d} 50) .\end{array}$}} \\
\hline Angle of Pipes $\left({ }^{\circ}\right)$ & 60 & & \\
\hline \multicolumn{4}{|c|}{ Overall Results } \\
\hline & $\begin{array}{c}\text { Total Mass of TSS Inflow } \\
(\mathrm{kg})\end{array}$ & $\begin{array}{l}\text { Total Mass being Trapped } \\
\qquad(\mathrm{kg})\end{array}$ & Total Removal Percentage (\%) \\
\hline Batch \#1 & \multirow{3}{*}{3.0} & 1.358 & $45.3 \%$ \\
\hline Batch \#2 & & 1.403 & $46.8 \%$ \\
\hline Batch \#3 & & 1.235 & $41.2 \%$ \\
\hline Mean & - & 1.332 & $44.4 \%$ \\
\hline Standard Deviation & - & 0.087 & 0.029 \\
\hline \multicolumn{4}{|c|}{ Discrete Results } \\
\hline Size: 710 microns & Mass of TSS Inflow $(\mathrm{kg})$ & Mass being Trapped (kg) & $\begin{array}{c}\text { Total Removal Percentage after } \\
\text { Correction }(\%) \\
\end{array}$ \\
\hline Batch \#1 & \multirow{3}{*}{0.15} & 0.1011 & $100.0 \%$ \\
\hline Batch \#2 & & 0.0836 & $100.0 \%$ \\
\hline Batch \#3 & & 0.0734 & $100.0 \%$ \\
\hline Mean & - & 0.0860 & $100.0 \%$ \\
\hline Standard Deviation & - & 0.0140 & 0.000 \\
\hline Size: 500 microns & Mass of TSS Inflow $(\mathrm{kg})$ & Mass being Trapped (kg) & $\begin{array}{c}\text { Total Removal Percentage after } \\
\text { Correction }(\%)\end{array}$ \\
\hline Batch \#1 & \multirow{3}{*}{0.15} & 0.3513 & $100.0 \%$ \\
\hline Batch \#2 & & 0.3571 & $100.0 \%$ \\
\hline Batch \#3 & & 0.2846 & $100.0 \%$ \\
\hline Mean & - & 0.3310 & $100.0 \%$ \\
\hline Standard Deviation & - & 0.0403 & 0.000 \\
\hline Size: 250 microns & Mass of TSS Inflow $(\mathrm{kg})$ & Mass being Trapped (kg) & $\begin{array}{c}\text { Total Removal Percentage after } \\
\text { Correction }(\%)\end{array}$ \\
\hline Batch \#1 & \multirow{3}{*}{0.45} & 0.3428 & \begin{tabular}{|c|}
$95.2 \%$ \\
\end{tabular} \\
\hline Batch \#2 & & 0.3494 & $93.6 \%$ \\
\hline Batch \#3 & & 0.3101 & $81.8 \%$ \\
\hline Mean & - & 0.3341 & $90.2 \%$ \\
\hline Standard Deviation & - & 0.0210 & 0.073 \\
\hline Size: 150 microns & Mass of TSS Inflow $(\mathrm{kg})$ & Mass being Trapped (kg) & $\begin{array}{c}\text { Total Removal Percentage after } \\
\text { Correction }(\%)\end{array}$ \\
\hline Batch \#1 & \multirow{3}{*}{0.45} & 0.2661 & $74.1 \%$ \\
\hline Batch \#2 & & 0.2778 & $77.1 \%$ \\
\hline Batch \#3 & & 0.2640 & $58.7 \%$ \\
\hline Mean & - & 0.2693 & $70.0 \%$ \\
\hline Standard Deviation & - & 0.0074 & 0.099 \\
\hline Size: 100 microns & Mass of TSS Inflow $(\mathrm{kg})$ & Mass being Trapped (kg) & $\begin{array}{c}\text { Total Removal Percentage after } \\
\text { Correction }(\%)\end{array}$ \\
\hline Batch \#1 & \multirow{3}{*}{0.30} & 0.1242 & $41.4 \%$ \\
\hline Batch \#2 & & 0.1410 & $47.0 \%$ \\
\hline Batch \#3 & & 0.1299 & $43.3 \%$ \\
\hline Mean & - & 0.1317 & $43.9 \%$ \\
\hline Standard Deviation & - & 0.0085 & 0.028 \\
\hline Size: 75 microns & Mass of TSS Inflow $(\mathrm{kg})$ & Mass being Trapped $(\mathrm{kg})$ & $\begin{array}{c}\text { Total Removal Percentage after } \\
\text { Correction }(\%)\end{array}$ \\
\hline Batch \#1 & \multirow{3}{*}{1.50} & 0.1484 & $9.9 \%$ \\
\hline Batch \#2 & & 0.1772 & $11.8 \%$ \\
\hline Batch \#3 & & 0.1570 & $10.5 \%$ \\
\hline Mean & - & 0.1609 & $10.7 \%$ \\
\hline Standard Deviation & - & 0.0148 & 0.010 \\
\hline
\end{tabular}




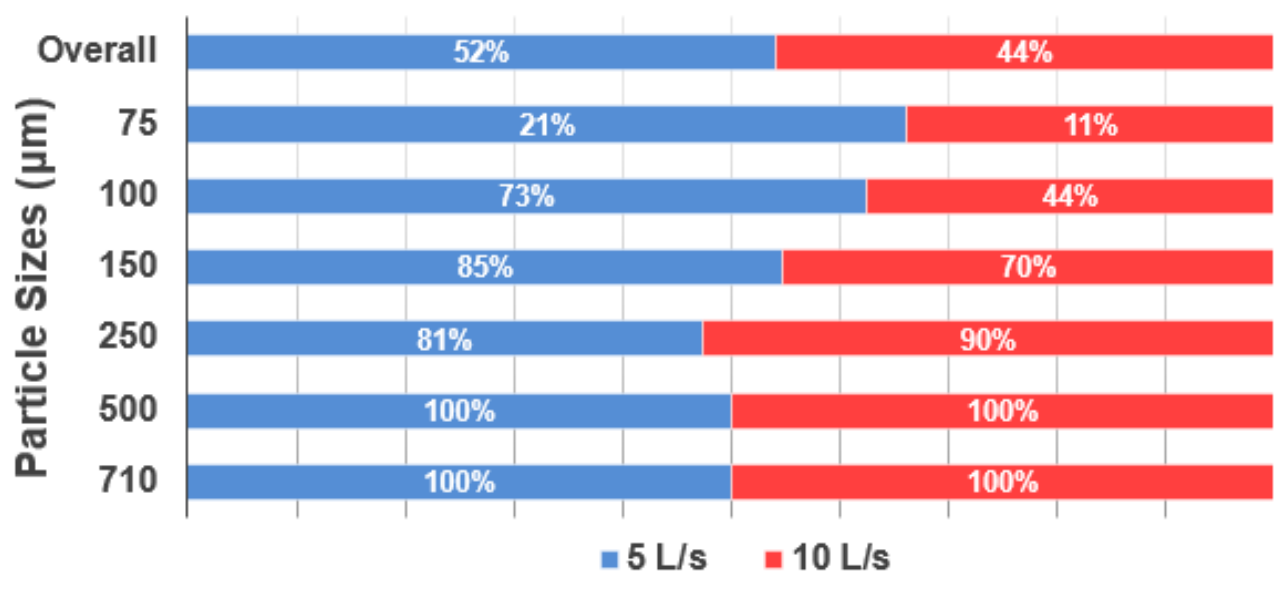

Figure 8 Proportional Comparison of Individually Sized Treatment Efficiencies between 5 and $10 \mathrm{~L} / \mathrm{s}$

From the results, the particle sized treatment efficiency was observed to decline with the particle size. As the flow rate doubled to $10 \mathrm{~L} / \mathrm{s}$, the overall TSS removal efficiency decreased from $52 \%$ to $44 \%$ (if excluding the data from Batch 5-3, a higher removal efficiency can be derived under 5 L/s flow with an overall performance of $54 \%$ instead of $52 \%$ ). In the meantime, the removal efficiency of $100-\mu \mathrm{m}$ particles dropped significantly (approximately $30 \%$ ), while that of $250-\mu \mathrm{m}$ particles increased slightly, which is, unexpectedly, contrary to the general trend.

\subsection{Evaluation and Comparison}

As stated in Chapter 2, technologies such as HDVS and OGS are both highly preferred due to the reasonable removal efficiency of sediments with a typical range of $40 \%$ to $60 \%$, while some can be higher. The claimed performance was further proved in a site performance assessment report prepared by SWAMP Program of TRCA in 2004. The program compared the most common types of Oil/Grit Separator products and found a typical range of $57 \%$ to $60 \%$ of TSS removal (TRCA - SWAMP Program, 2004). 
This research has demonstrated that the experimental vortex device has an overall TSS removal efficiency of $40 \%$ to $50 \%$ and $50 \%$ to $55 \%$ under $5 \mathrm{~L} / \mathrm{s}$ and $10 \mathrm{~L} / \mathrm{s}$ of flows respectively, which are within the typical treatment range of HDVS and OGS. However, higher requirements toward TSS removal efficiency are found in many environmental-related regulatory acts. For instance, according to "Wet Weather Flow Management Guidelines" prepared by city of Toronto, although OGS devices are acceptable for achieving a TSS removal efficiency of 50\%, "the wet weather flow (WWF) water quality target is the long-term average removal of $80 \%$ of total suspended solids (TSS) based on an annual loading basis from all runoff leaving the proposed development site based on the post-development level of imperviousness." (City of Toronto, 2006). As a result, enhanced treatment methods such as filtration technology - "Jellyfish® Filter" (Imbrium Systems Inc.), or multiple LID practices in-series shall be implemented together with OGS/HDVS to achieve a better stormwater quality.

\subsection{Summary}

Although this research has tested only two flow rates, the results indicate that the overall TSS treatment efficiency of EVD is about $50 \%$ and decreases as the flow rate increases. As a result, technologies like EVD in this research is recommended to be implemented along with other LID practices or methods in order to fulfill the normal required TSS treatment level (e.g., 80\%) in many jurisdictions. 


\subsection{CONCLUSIONS AND RECOMMENDATIONS}

\subsection{Conclusions}

Based on the findings of this research project, the following conclusions are drawn:

1. Vortex separation has been successfully employed to remove suspended solids from stormwater. The combined centrifugal and gravity forces induced by spherical circulation of water increase solids and oil separation, resulting in a small surface area of separation. Technologies which employ this vortex separation are particularly suitable for treatment of stormwater in highly urbanized areas where the lack of space restricts the employment of other stormwater management measures such as stormwater ponds.

2. The experimental vortex device (EVD) was designed as an off-line treatment unit using a flow diversion baffle wall at the upstream manhole. In addition, the central outflow pipe coupled with an arch baffle wall was sized to allow the sedimentation of suspended solids and floatation of oil inside the EVD, and the bypass of flows larger than the design treatment rate. Based on the target catchment area in Tianjin, the full depth of $1.8 \mathrm{~m}$ of the $1.2 \mathrm{~m}$ device can provide sufficient capacity for sedimentation, floatation, and sediment storage.

3. The Procedure for Laboratory Testing of Oil/ Grit Separators (TRCA 2014) specifies a comprehensive laboratory procedure to determine the solids and oil removal performance of oil/grit separators. This research has found several challenges and potential solutions: (a) In order to consistently discharge the set amount sediments over the test duration of 25 minutes, the mechanical input mechanism must be calibrated; (b) a representative sampling of influent TSS concentration downstream of the sediment input location (e.g. 5 times the diameter of the influent pipe) may be difficult while the sampling at the influent pipe outlet may offer an alternative location; (c) collection of the trapped sediments at the bottom of 
EVD (by installing a sloping base with the sludge outlet pipe located at the middle) is more practical than that at the EVD's outlet; (d) a flow-recirculation system is preferable to a 'continuous feeding-and-wasting' system without recirculation for the physical model because wastage of water can be significant (flow rate grows exponentially); (e) if a flowrecirculation system is employed, the effluent from the outlet of the EVD should be discharged to a turbid pond where a separating wall with a weir and filter screen (mesh size corresponds to the finest sediments used in experiments) can be used to trap the background effluent sediment before entering a clean pond for the influent intake; (f) transparent materials (e.g. plexiglass), which allow visualization of flow patterns, sedimentation, and floatation, should be used to construct the physical model; and (g) a rigorous daily laboratory working schedule as shown in Appendix A can ensure the best use of time for the experiments.

4. The average TSS removal efficiency of the EVD at 5 and $10 \mathrm{~L} / \mathrm{s}$ is about $50 \%$ which is comparable to other similar OGS devices. It decreased from $52 \%$ to $44 \%$ as the flow rate was doubled from $5 \mathrm{~L} / \mathrm{s}$ to $10 \mathrm{~L} / \mathrm{s}$.

\subsection{Recommendations}

After completing this research, recommendations of future works are:

1. Since this project only tested $5 \mathrm{~L} / \mathrm{s}$ and $10 \mathrm{~L} / \mathrm{s}$, higher flow rates up to and exceeding the maximum treatment rate should be applied to the device to determine the TSS removal efficiency and its trend. 
2. Minor alternations of the EVD configuration (e.g. the horizontal angle between the influent and effluent, the slope of the influent pipe, the height of the central outflow pipe) can be used to optimize the TSS removal efficiency.

3. The velocities and water depths measured during experiments should be used to calibrate a CFD model for the simulation of optimized configurations of the EVD.

4. Oil and water separation at the EVD should be investigated using plastic beads (with relative density similar to the target oil interception). 


\section{Appendix A}

- 08:30 - Start with preparation works such as model clean-up and filling;

- 09:30 - Adjust all the equipment and instruments;

- 10:00 - Initiate the pump, wait for the water level to be stabilized;

- 10:10 - Start the first physical testing and let the system running for $25 \mathrm{mins}$;

- 10:35 - Stop the pump and all instruments, let the system rest for $15 \mathrm{mins}$, and meanwhile organize all the collected data;

- 10:50 - Drain out the water in the device, retrieve the first batch of trapped residual;

- 11:30 - Drying the residual;

- Lunch Break;

- 14:00 - Collect the dried residual and send to the analyzing lab;

- 14:30 - Filling up the model;

- 14:50 - Adjust some equipment and instruments;

- 15:05 - Initiate the pump, wait for the water level to be stabilized;

- 15:15 - Start the second physical testing and let the system running for $25 \mathrm{mins}$;

- 15:40 - Stop the pump and all instruments, let the system rest for $15 \mathrm{mins}$, and meanwhile organize all the collected data;

- 15:55 - Drain out the water in the device, retrieve the second batch of trapped residual;

- $16: 35$ - Drying the second batch of residual;

- 17:00 - Get off work.

(Repeating the schedule) 
Appendix B

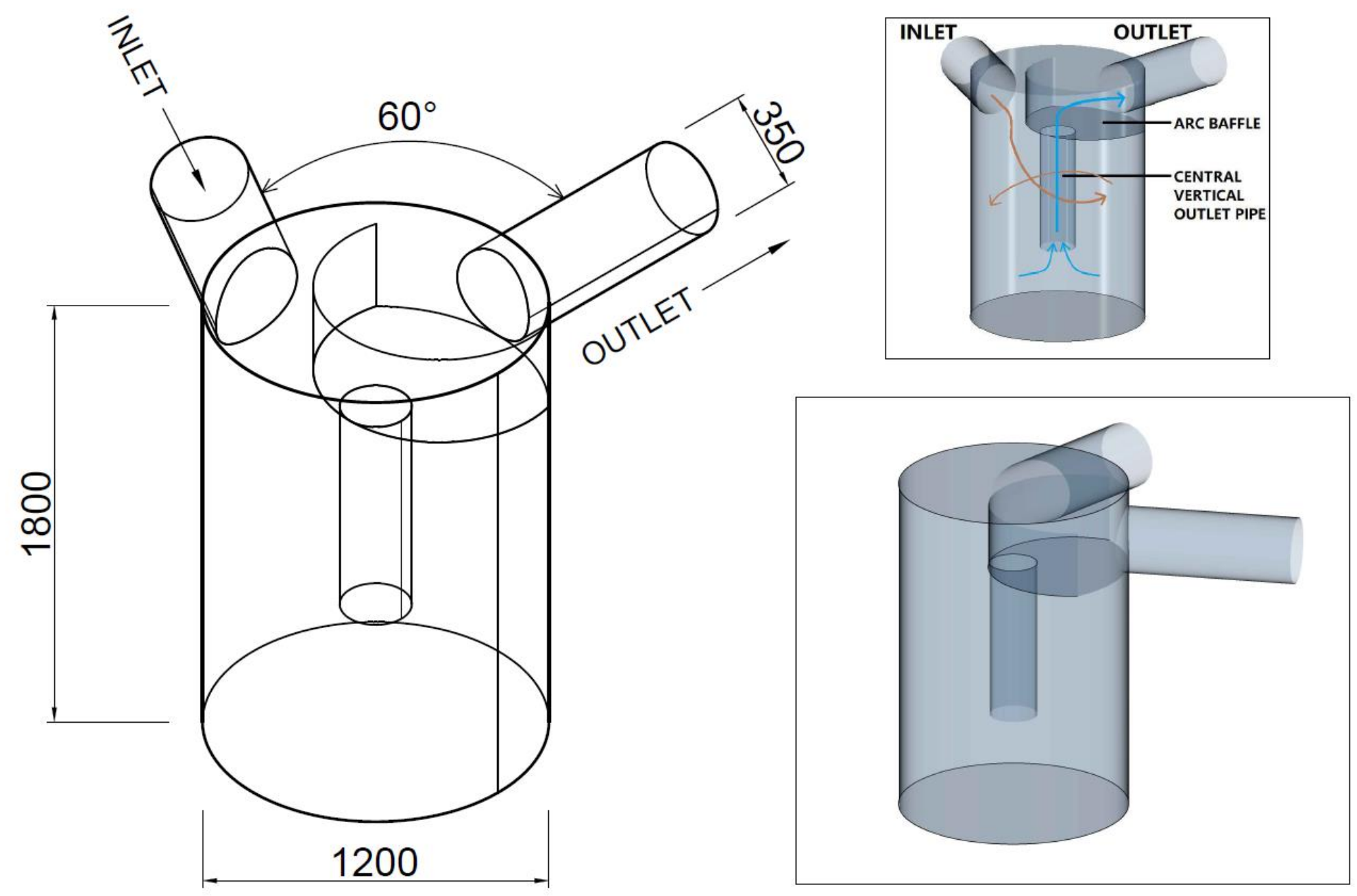




\section{Appendix C}

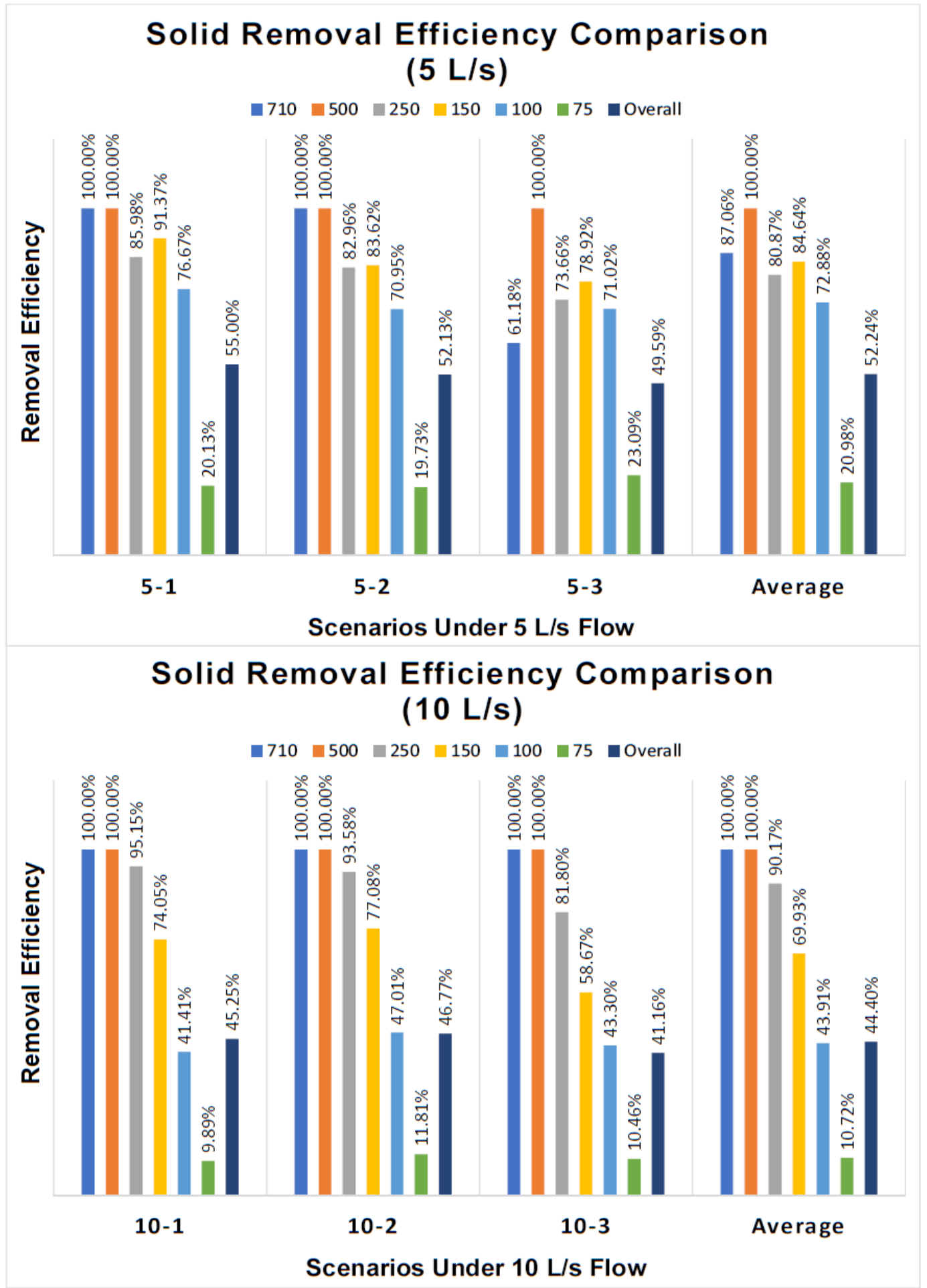




\section{Appendix D}

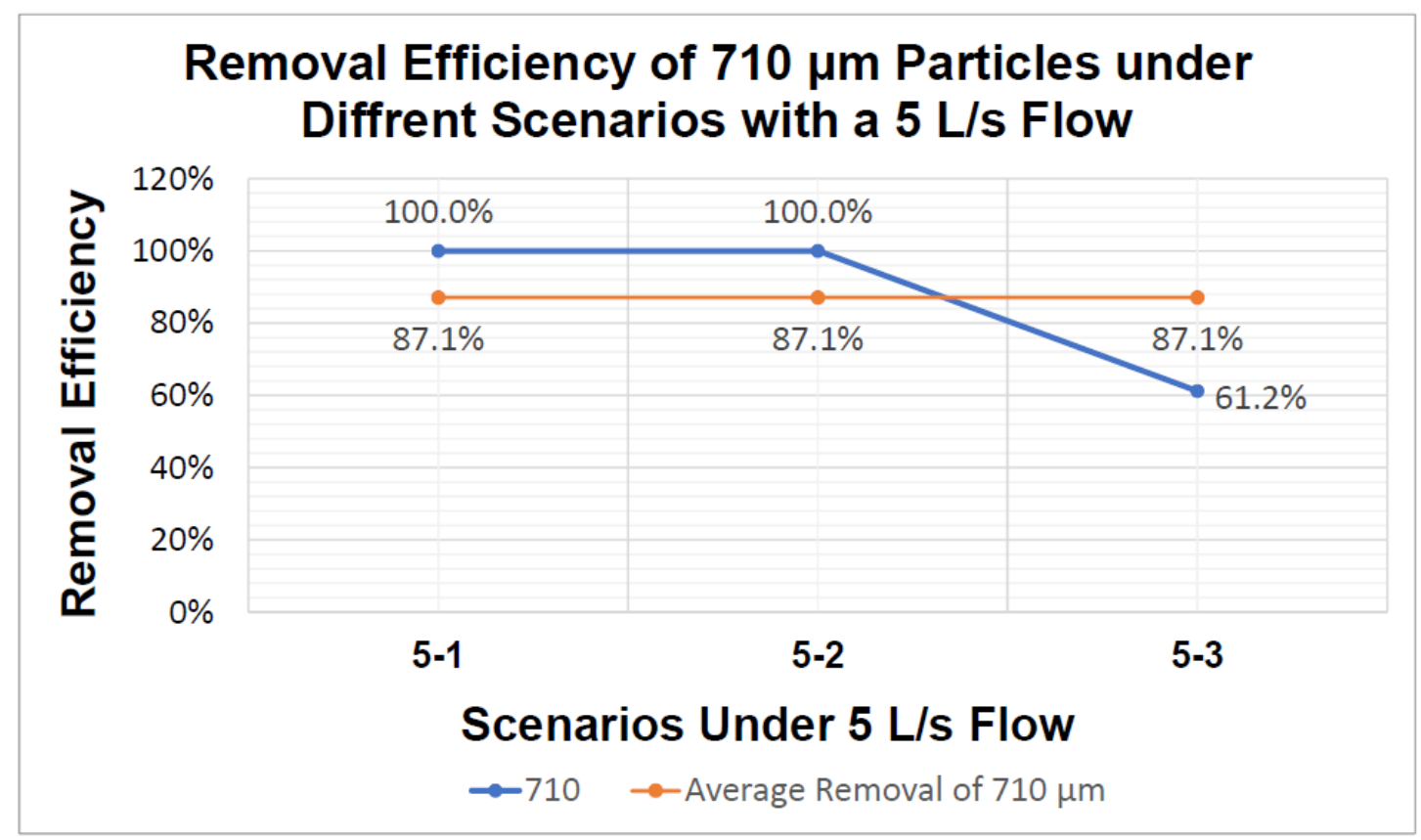

Appendix D. 1 Removal Efficiency of 710-Micron Particles $(5 \mathrm{~L} / \mathrm{s})$

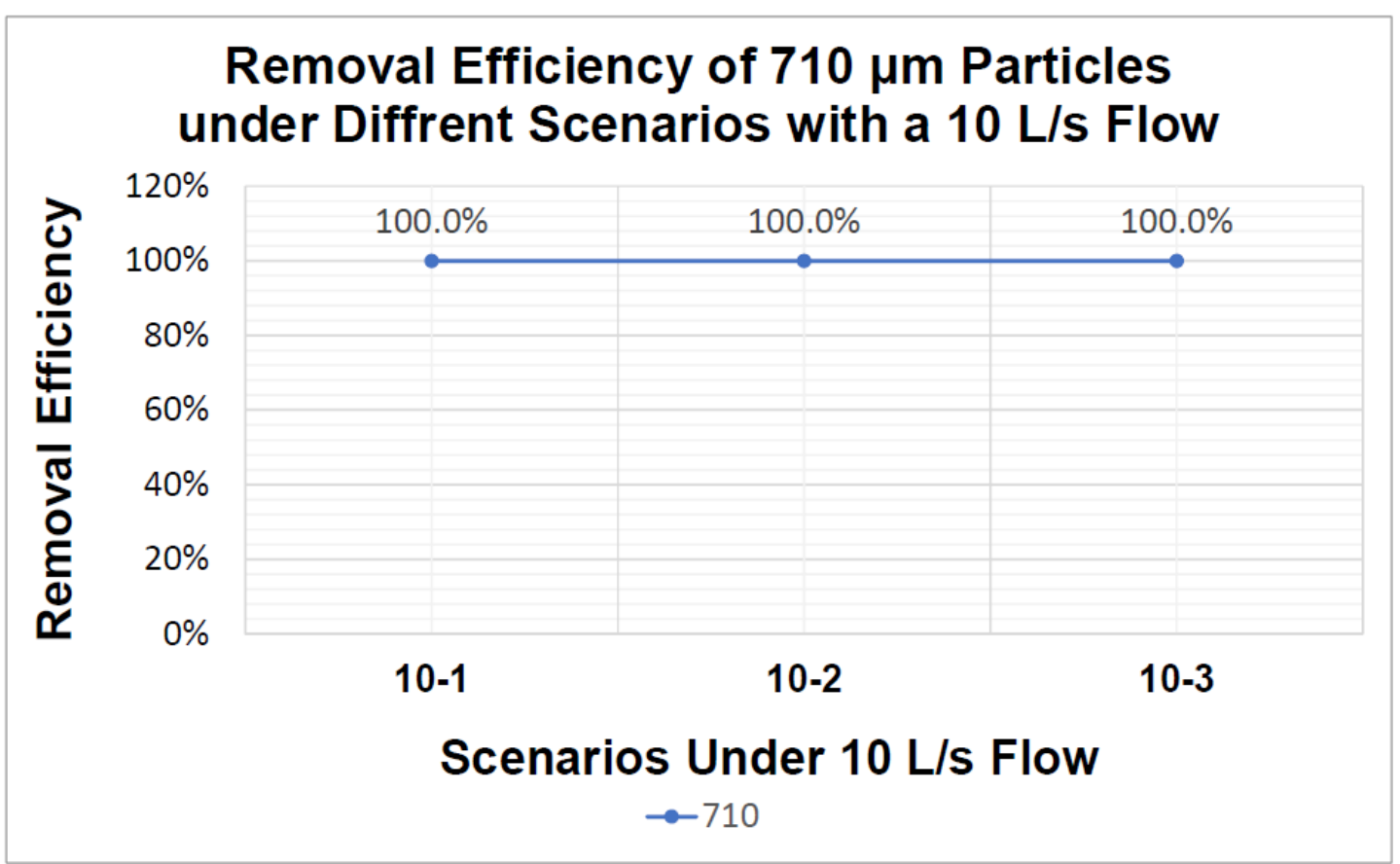

Appendix D. 2 Removal Efficiency of 710-Micron Particles $(10 \mathrm{~L} / \mathrm{s})$ 


\section{Appendix D - Cont'd}

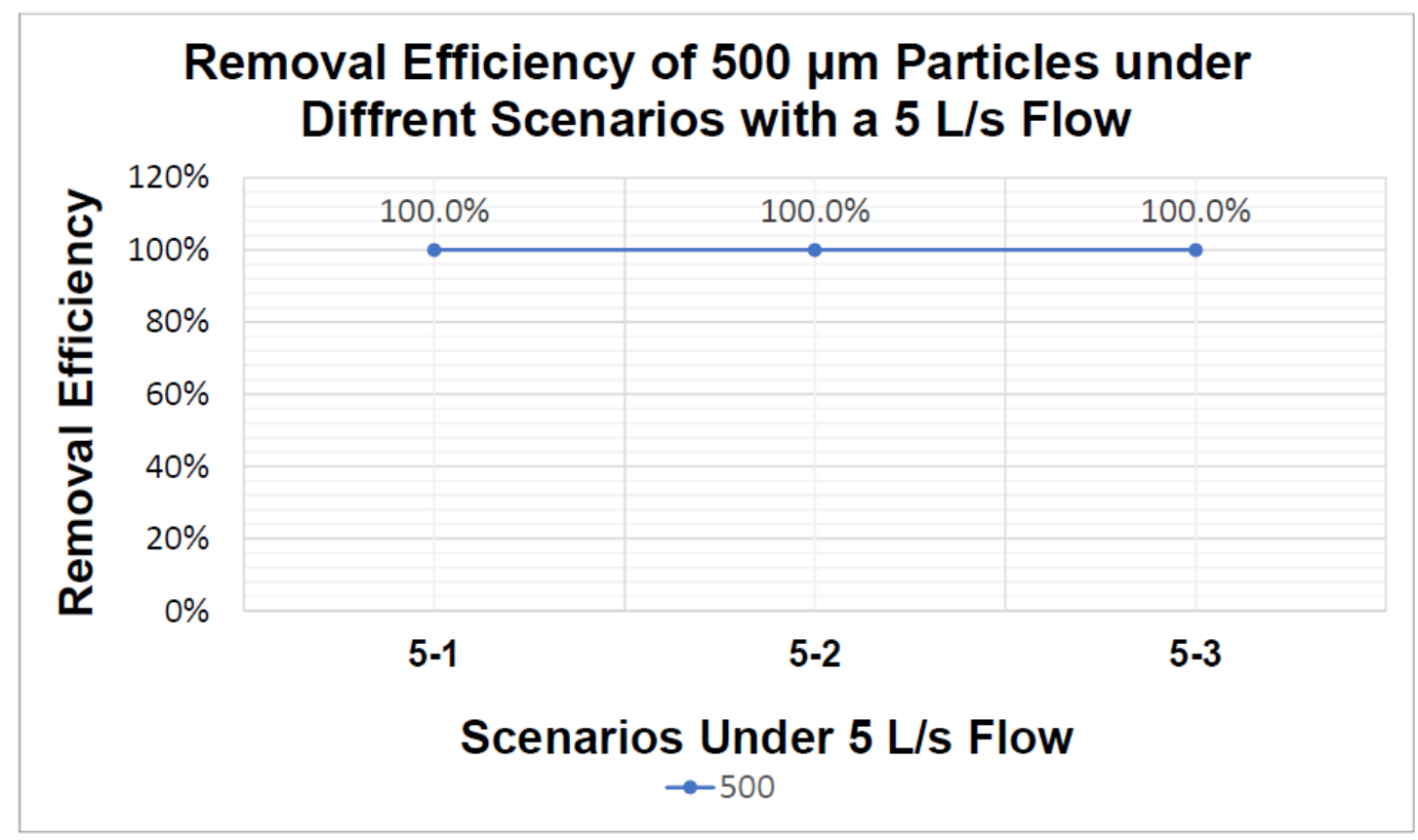

Appendix D. 3 Removal Efficiency of 500-Micron Particles $(5 \mathrm{~L} / \mathrm{s})$

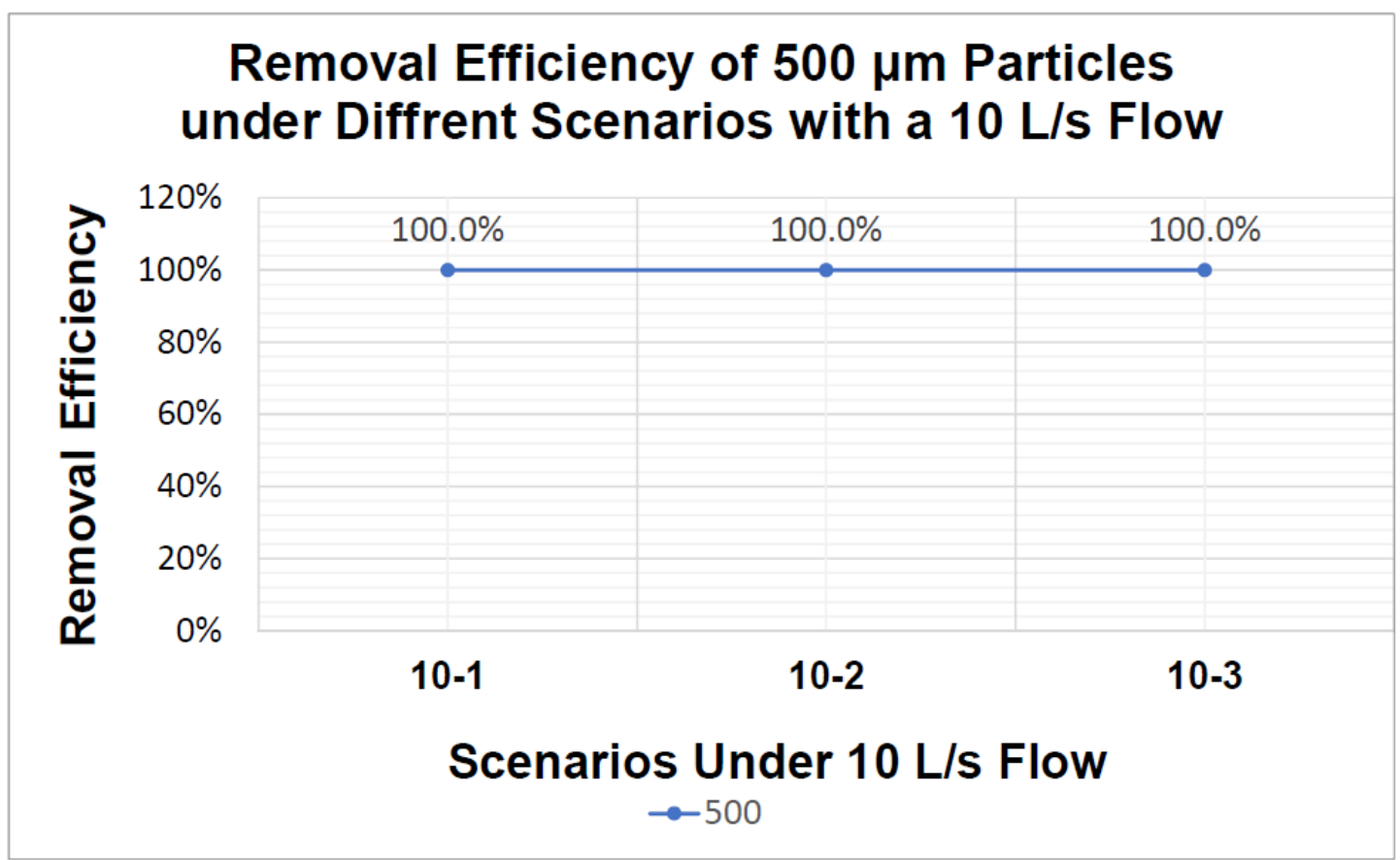

Appendix D. 4 Removal Efficiency of 500-Micron Particles $(10 \mathrm{~L} / \mathrm{s})$ 


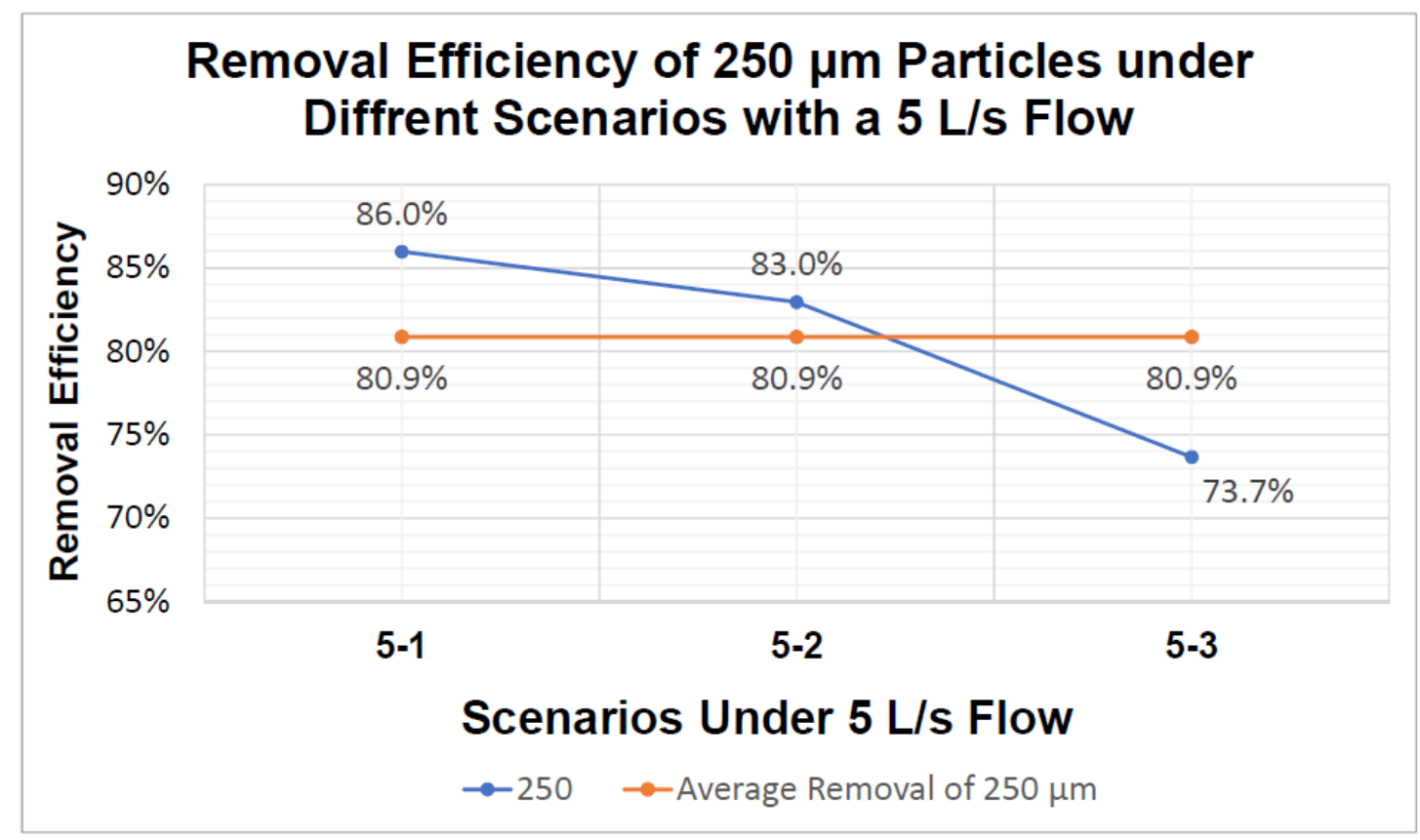

Appendix D. 5 Removal Efficiency of 250-Micron Particles $(5 \mathrm{~L} / \mathrm{s})$

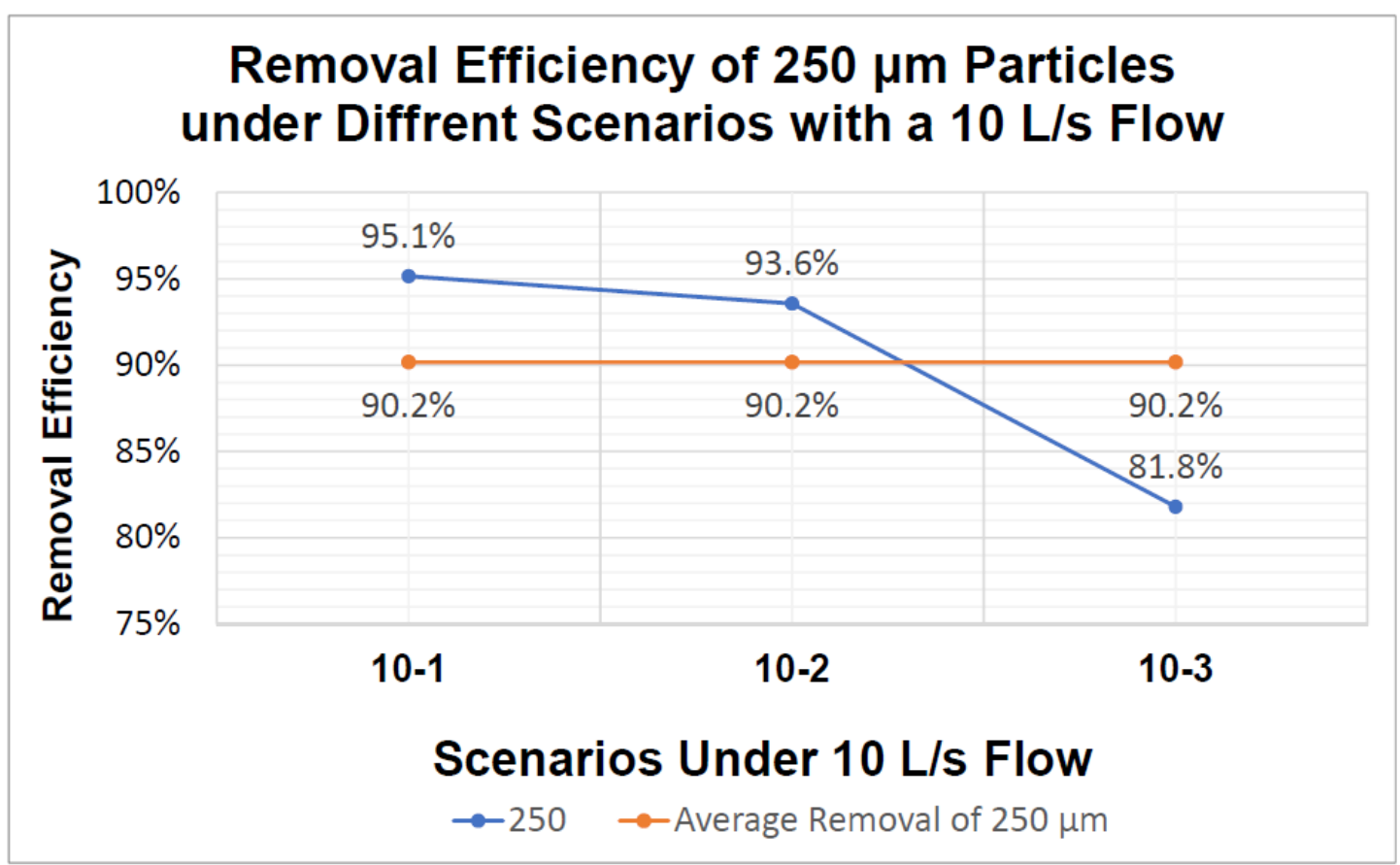

Appendix D. 6 Removal Efficiency of 250-Micron Particles $(10 \mathrm{~L} / \mathrm{s})$ 


\section{Appendix D - Cont'd}

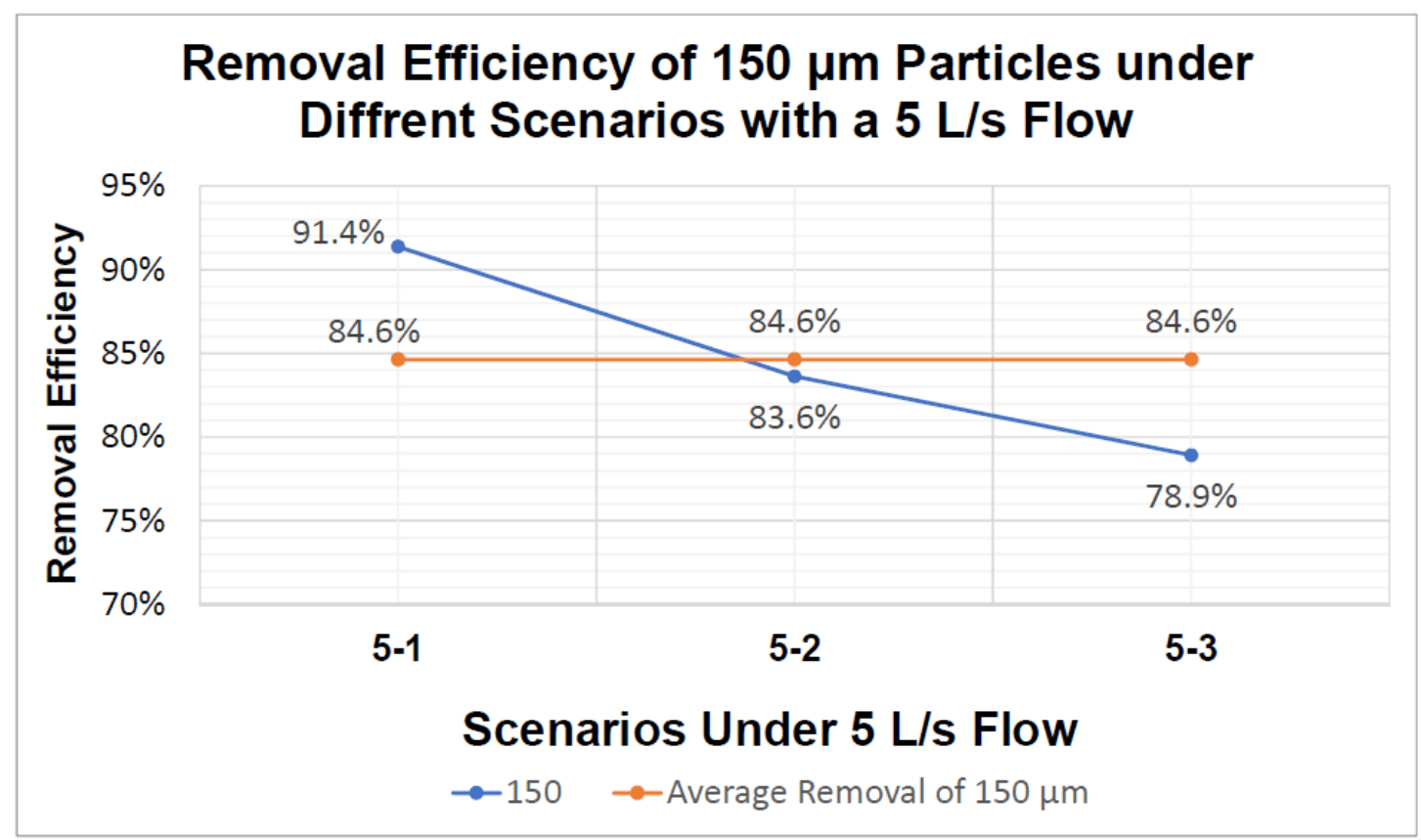

Appendix D. 7 Removal Efficiency of 150-Micron Particles $(5 \mathrm{~L} / \mathrm{s})$

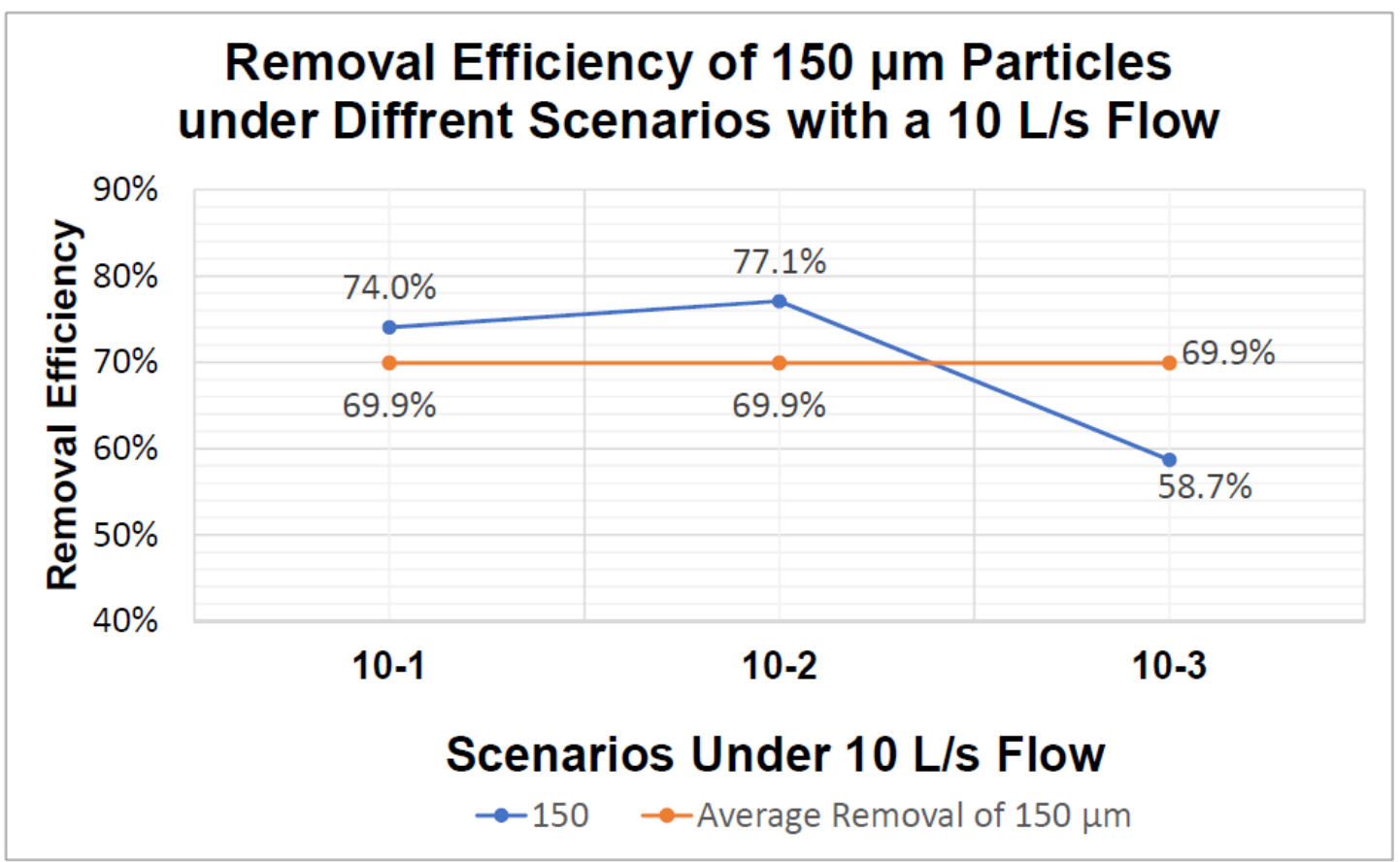

Appendix D. 8 Removal Efficiency of 150-Micron Particles $(10 \mathrm{~L} / \mathrm{s})$ 


\section{Appendix D - Cont'd}

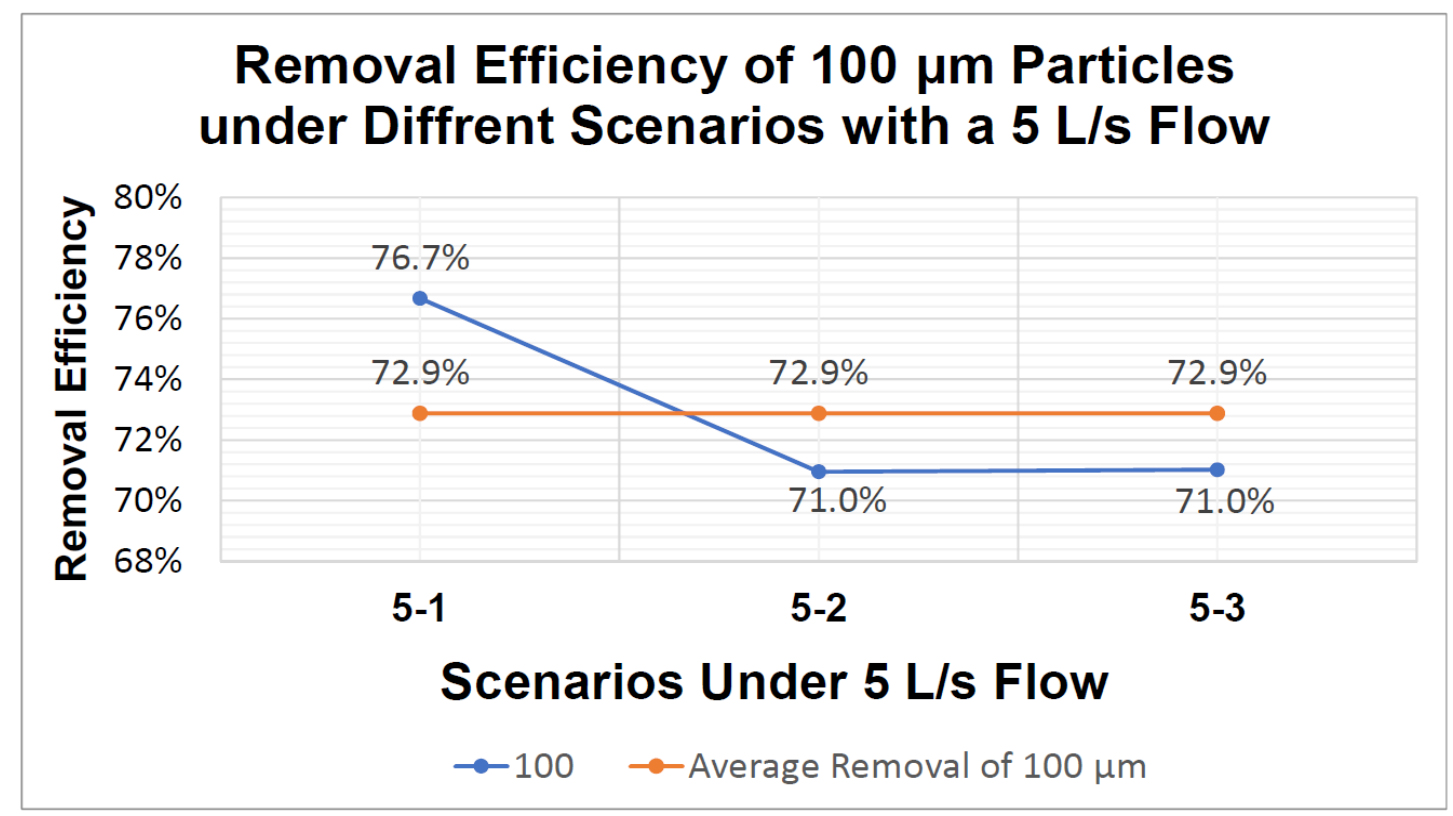

Appendix D. 9 Removal Efficiency of 100-Micron Particles $(5 \mathrm{~L} / \mathrm{s})$

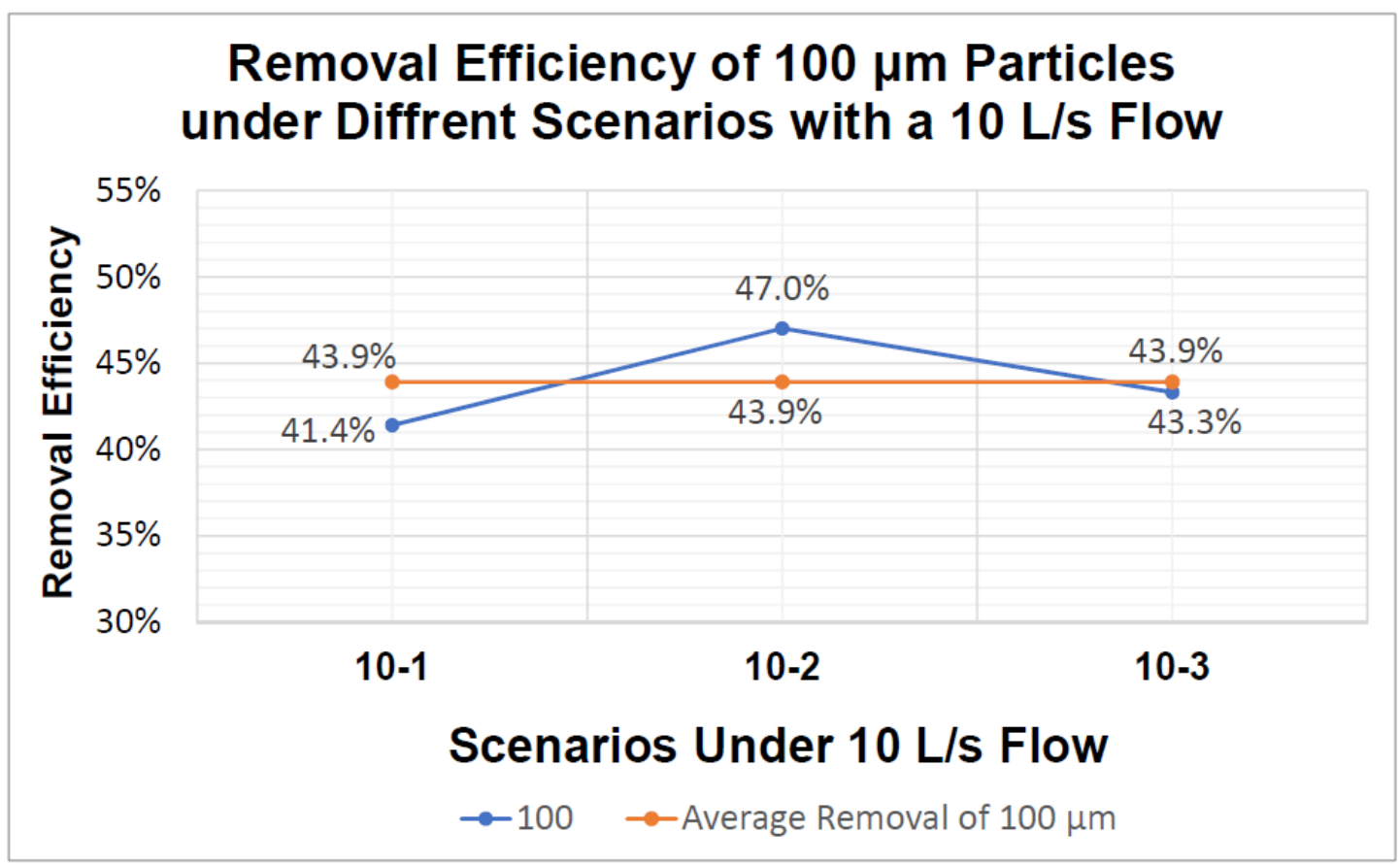

Appendix D. 10 Removal Efficiency of 100-Micron Particles $(10 \mathrm{~L} / \mathrm{s})$ 


\section{Appendix D - Cont'd}

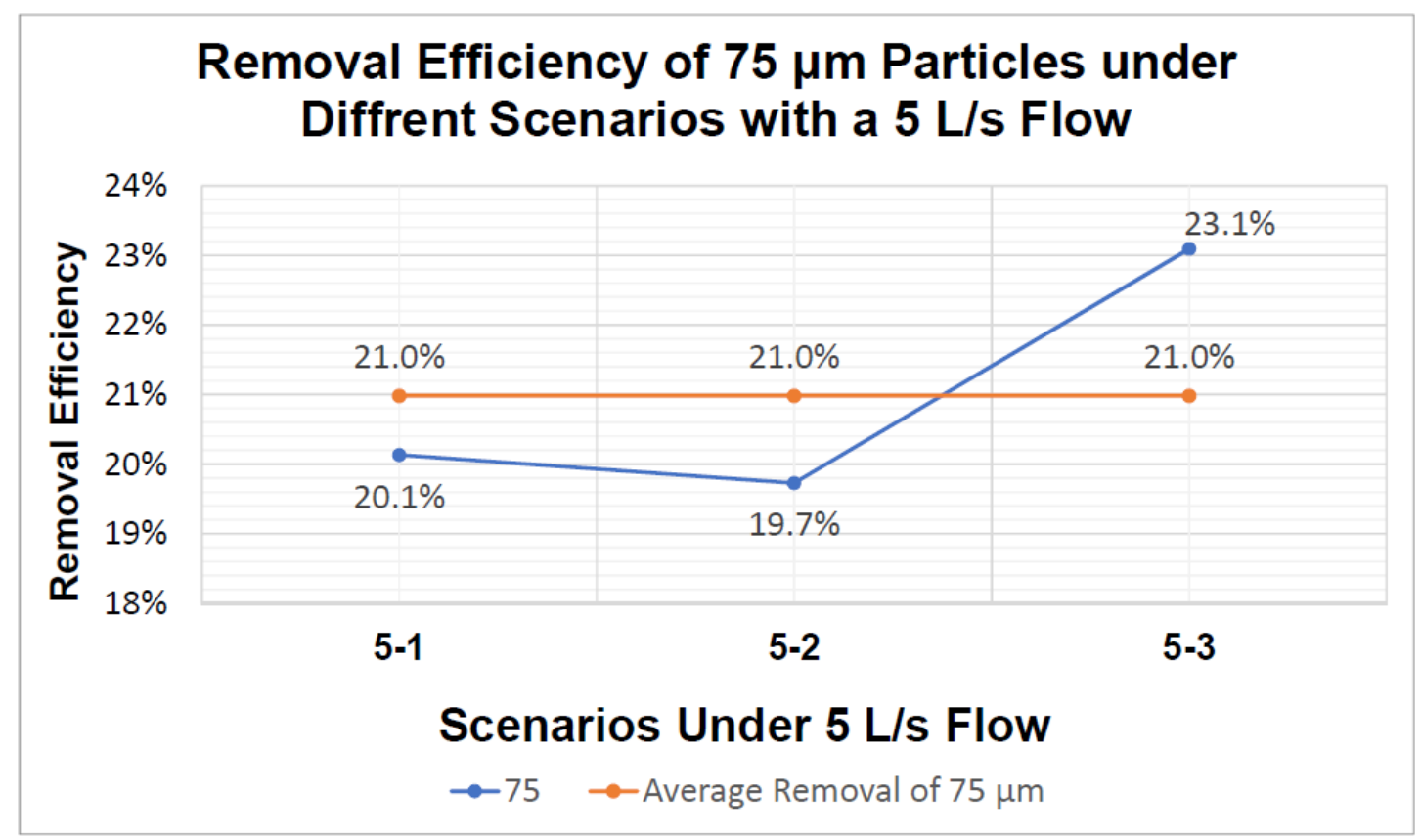

Appendix D. 11 Removal Efficiency of 75-Micron Particles $(5 \mathrm{~L} / \mathrm{s})$

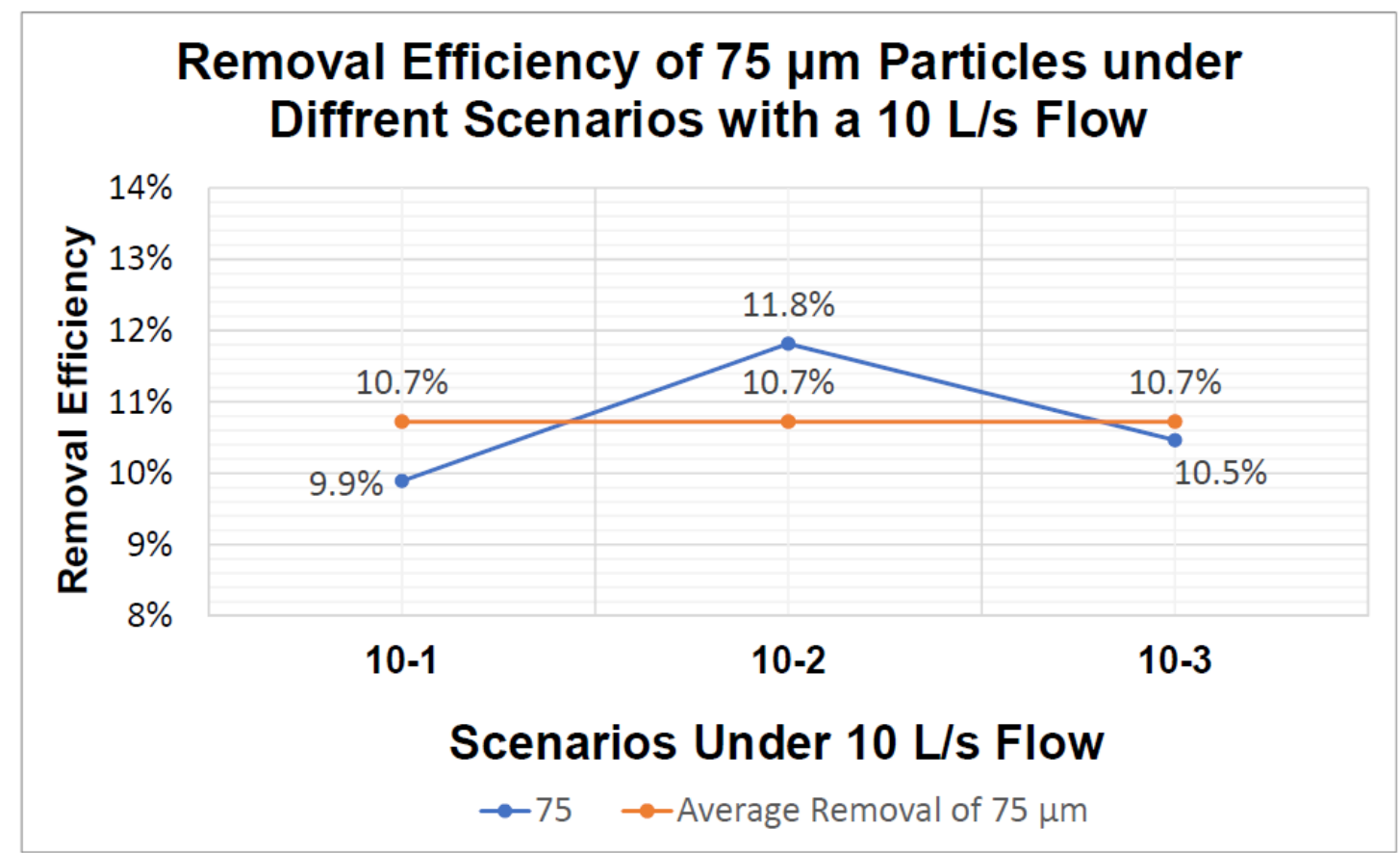

Appendix D. 12 Removal Efficiency of 75-Micron Particles $(10 \mathrm{~L} / \mathrm{s})$ 


\section{Appendix D - Cont'd}

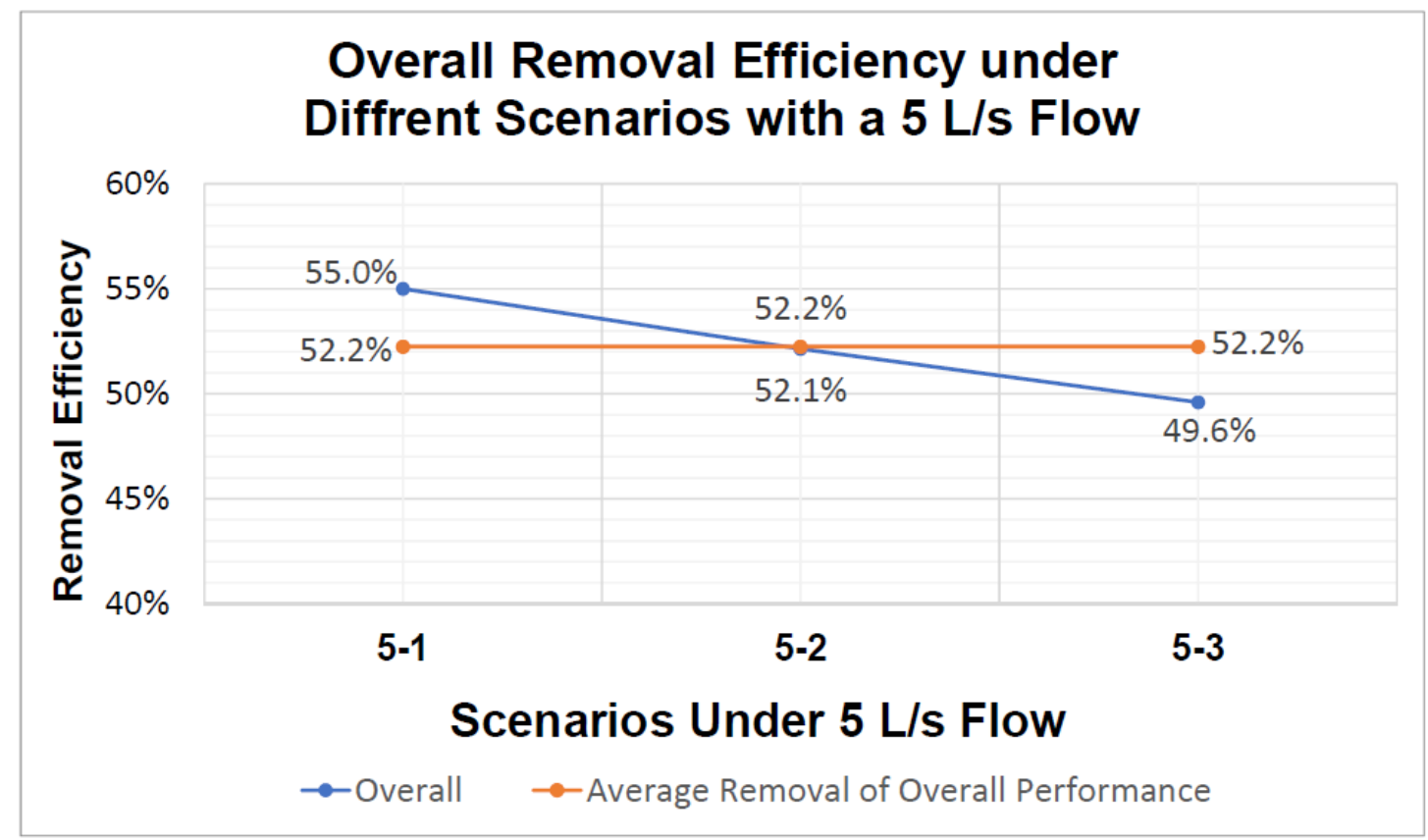

Appendix D. 13 Removal Efficiency of Overall Performance $(5 \mathrm{~L} / \mathrm{s})$

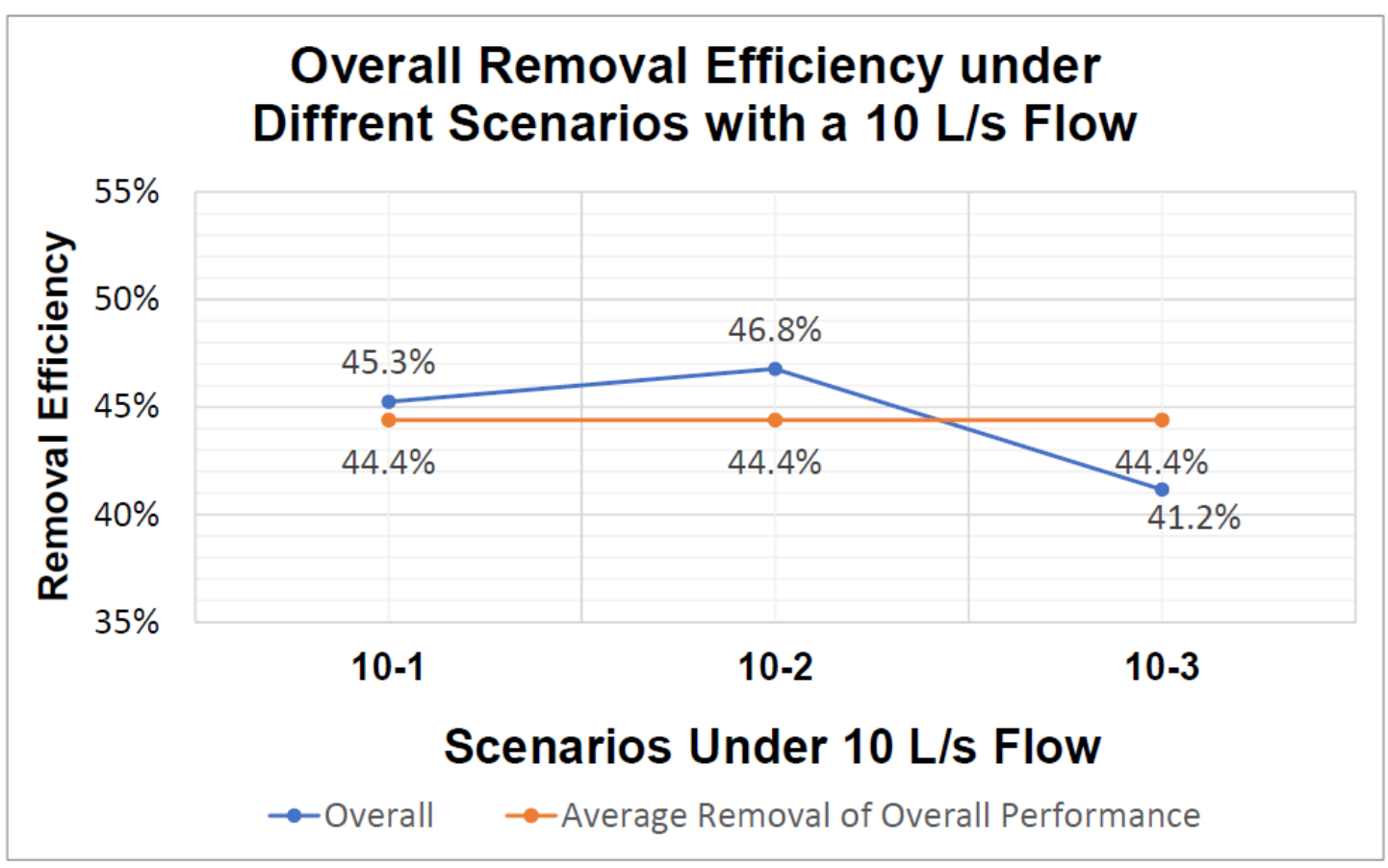

Appendix D. 14 Removal Efficiency of Overall Performance $(10 \mathrm{~L} / \mathrm{s})$ 


\section{Appendix E}

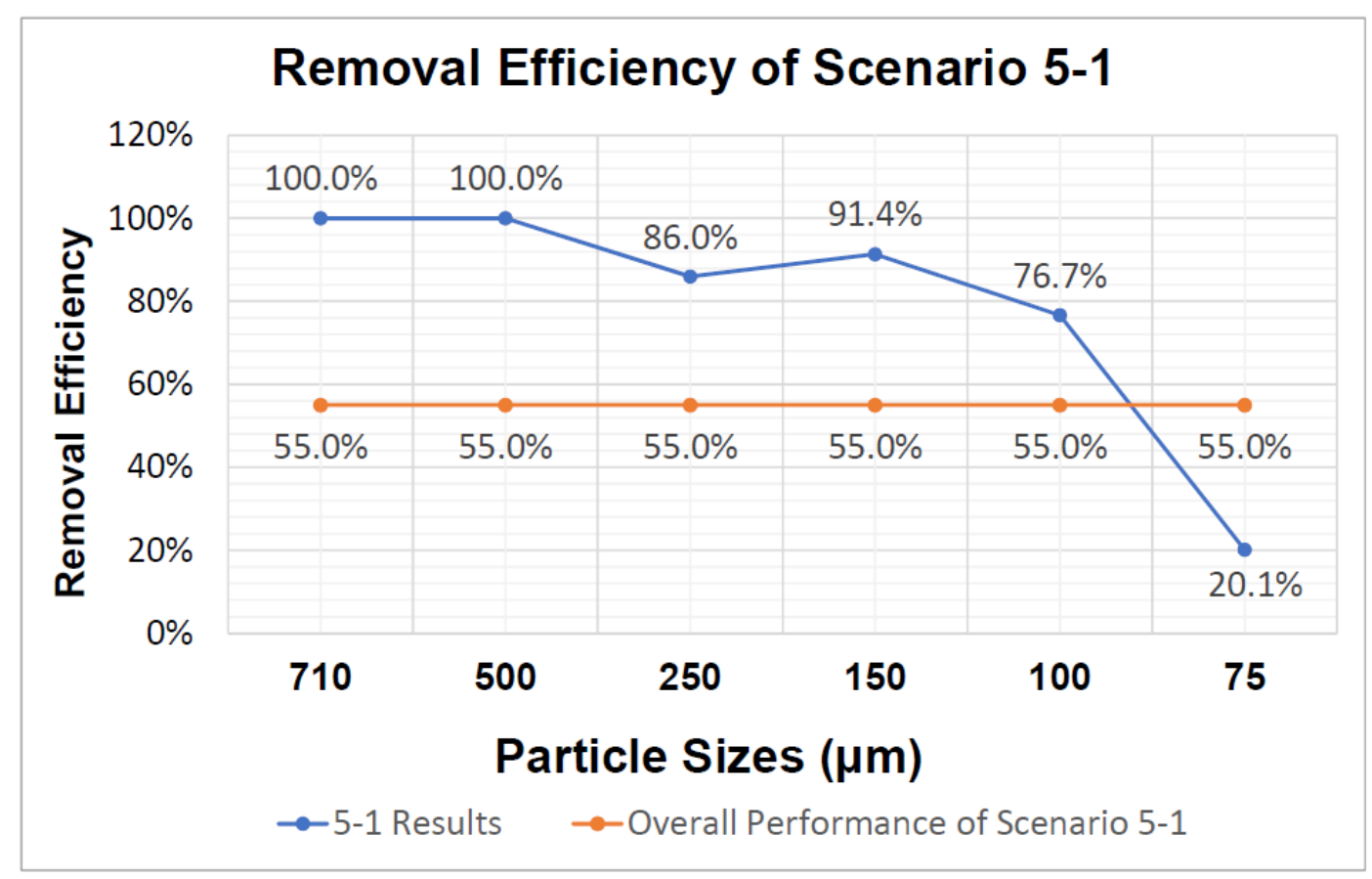

Appendix E. 1 Removal Efficiency of Scenario 5-1

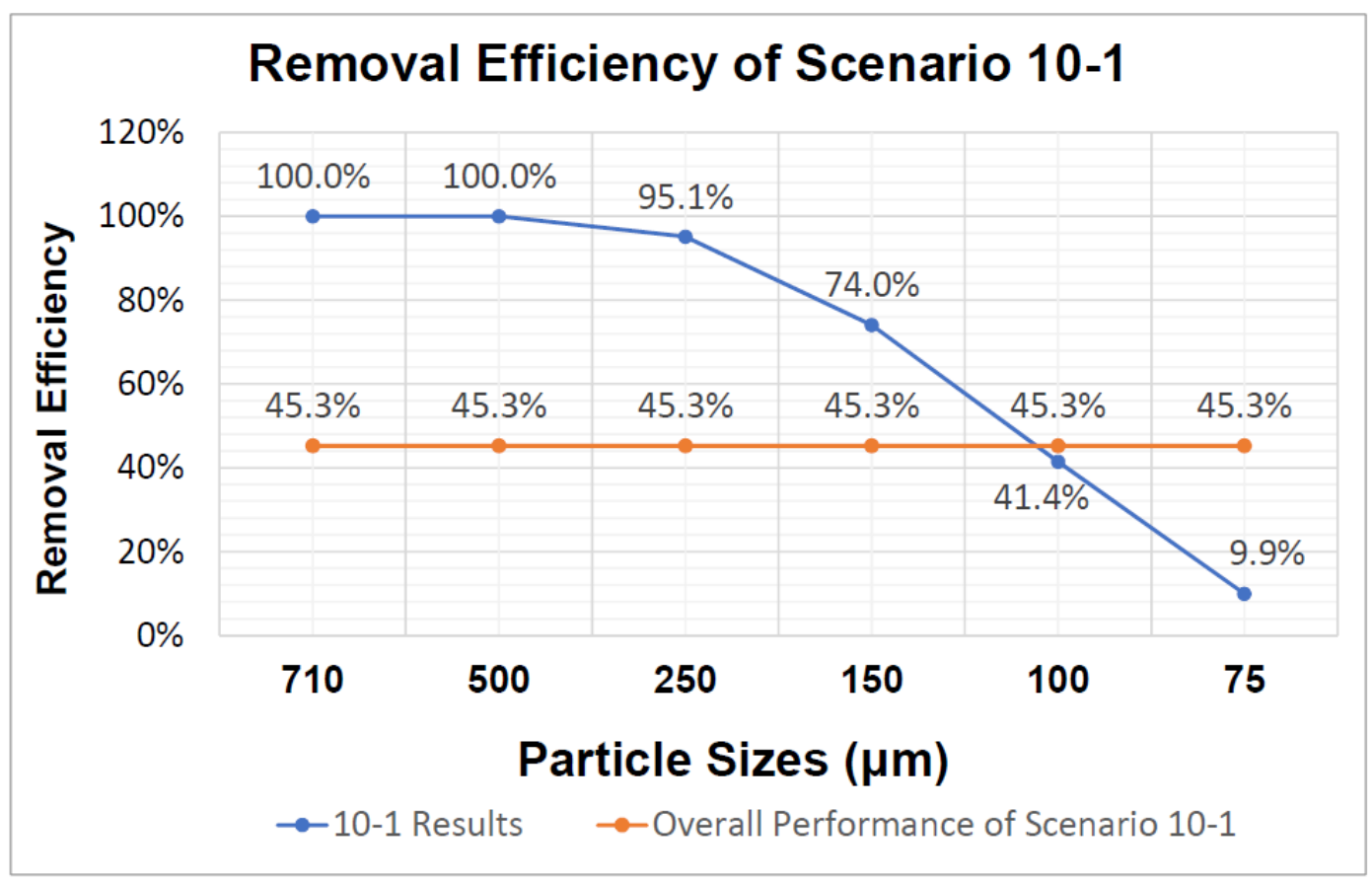

Appendix E. 2 Removal Efficiency of Scenario 10-1 


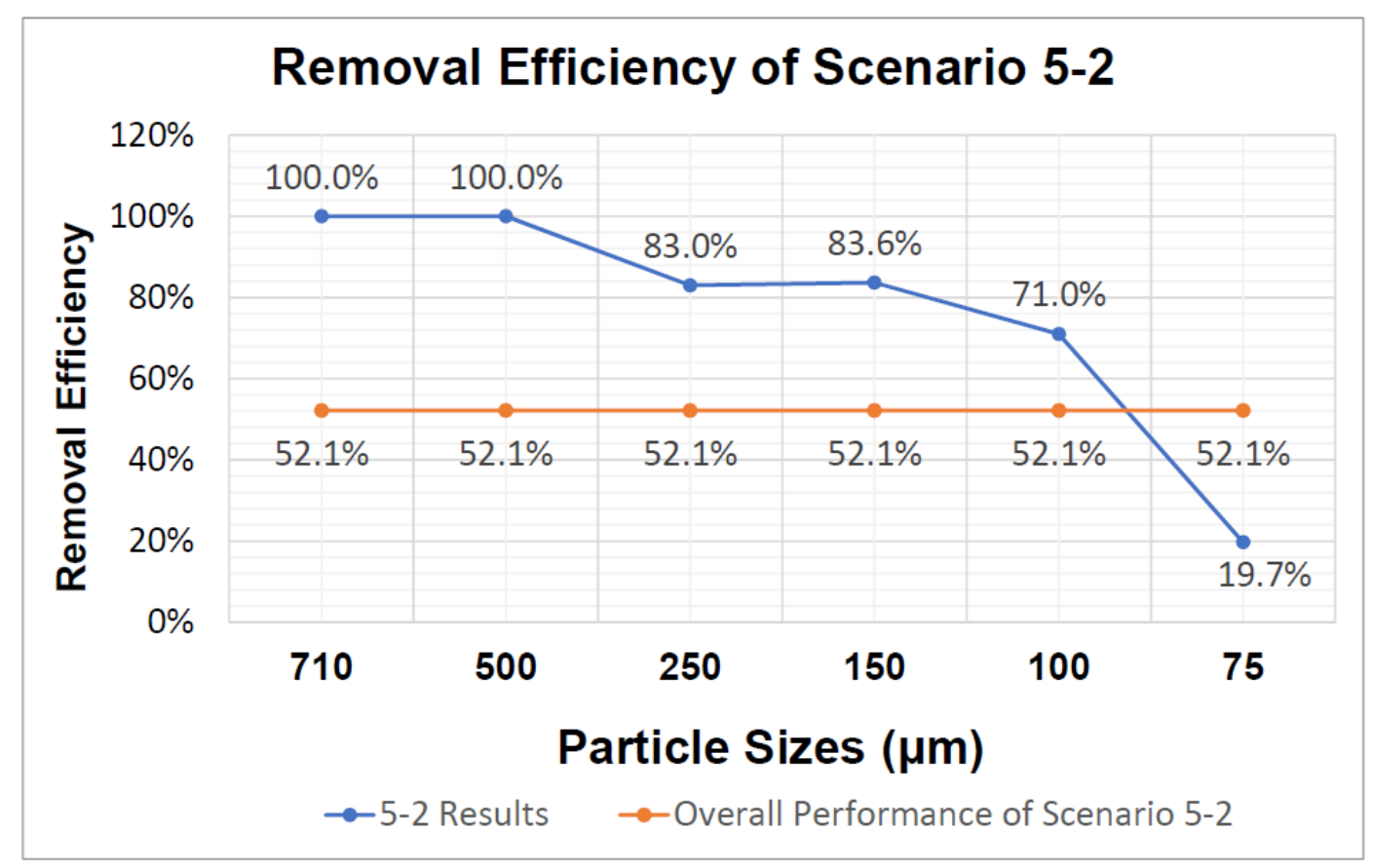

Appendix E. 3 Removal Efficiency of Scenario 5-2

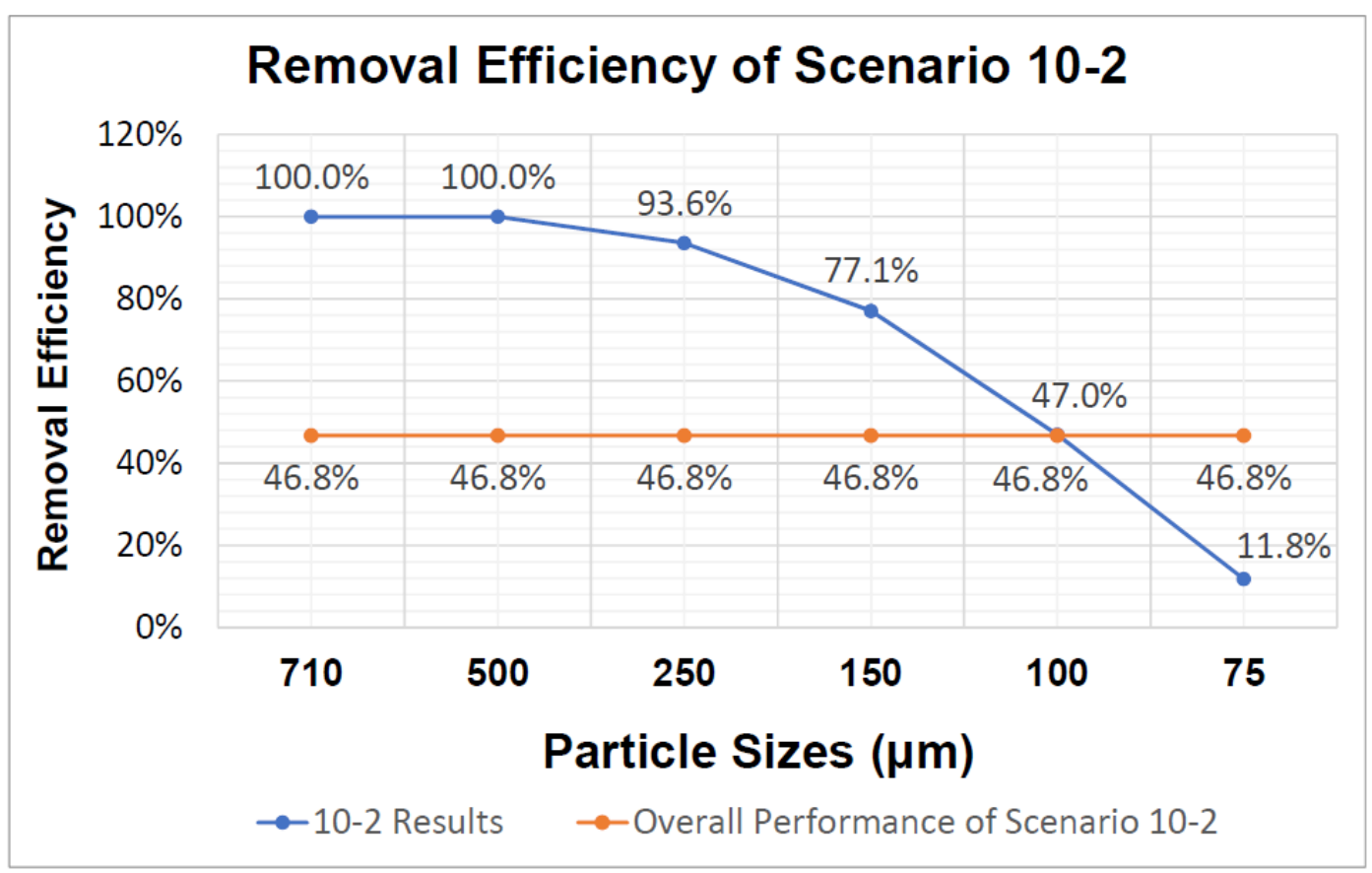

Appendix E. 4 Removal Efficiency of Scenario 10-2 


\section{Appendix E - Cont'd}

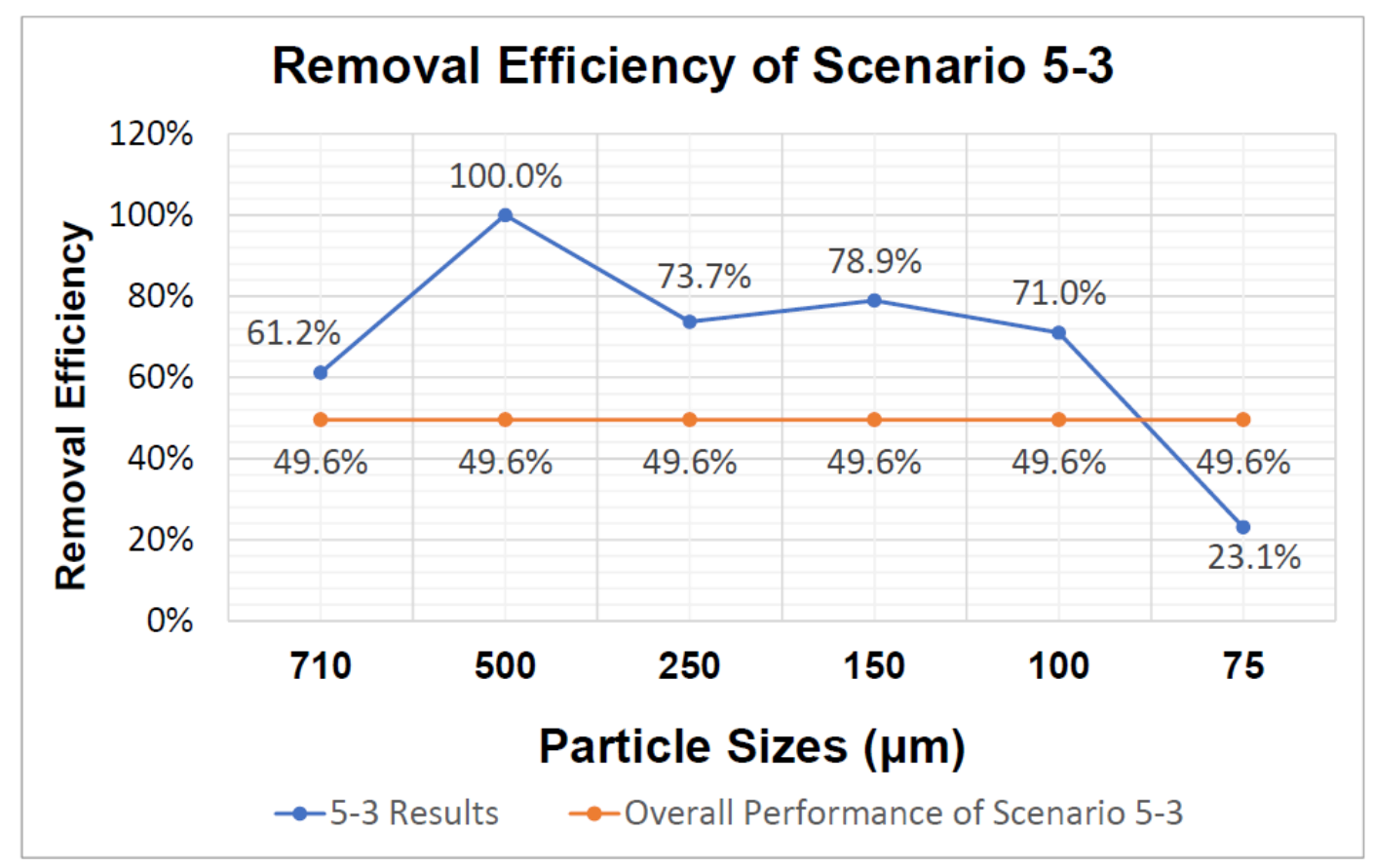

Appendix E. 5 Removal Efficiency of Scenario 5-3

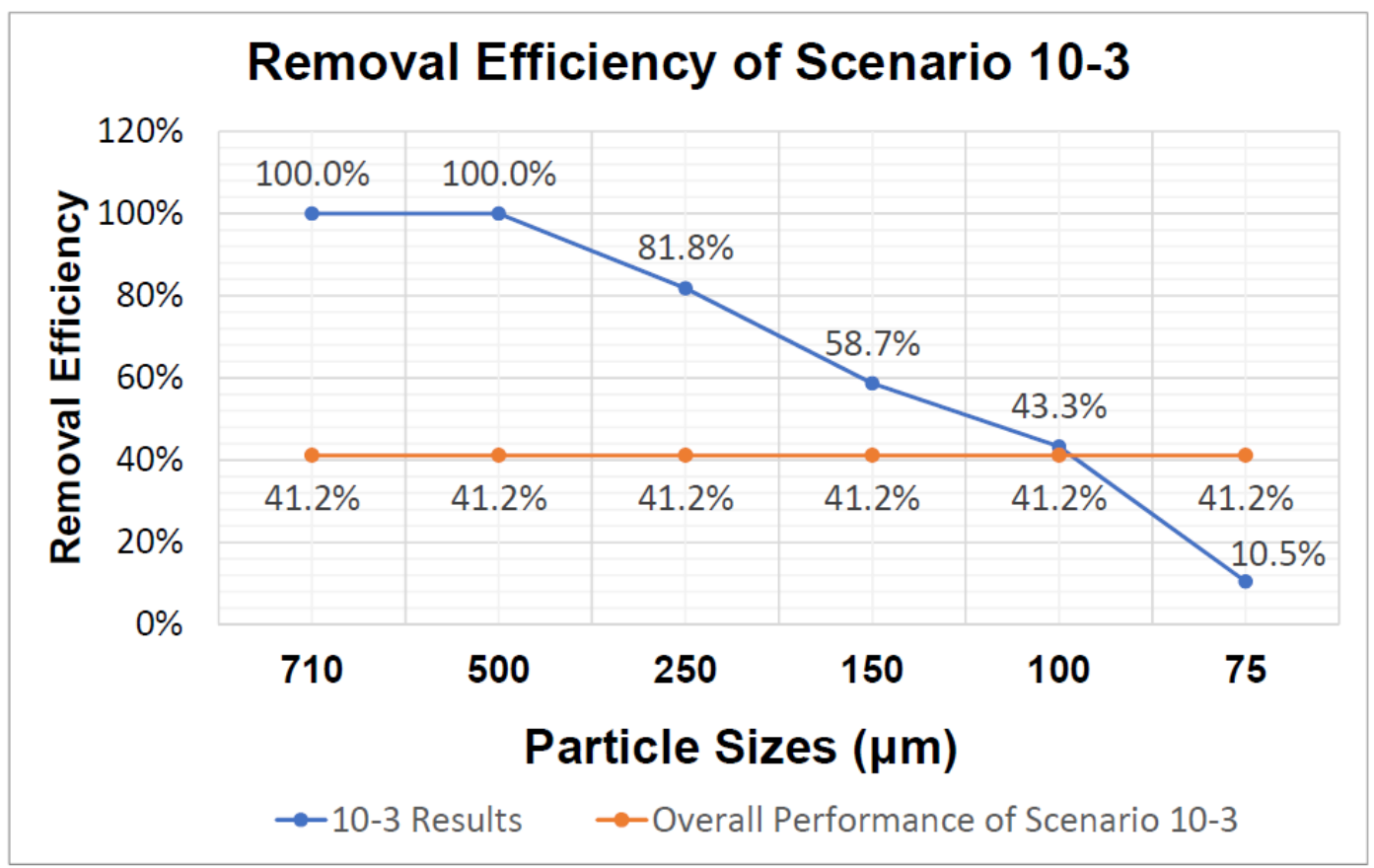

Appendix E. 6 Removal Efficiency of Scenario 10-3 


\section{Appendix E - Cont'd}

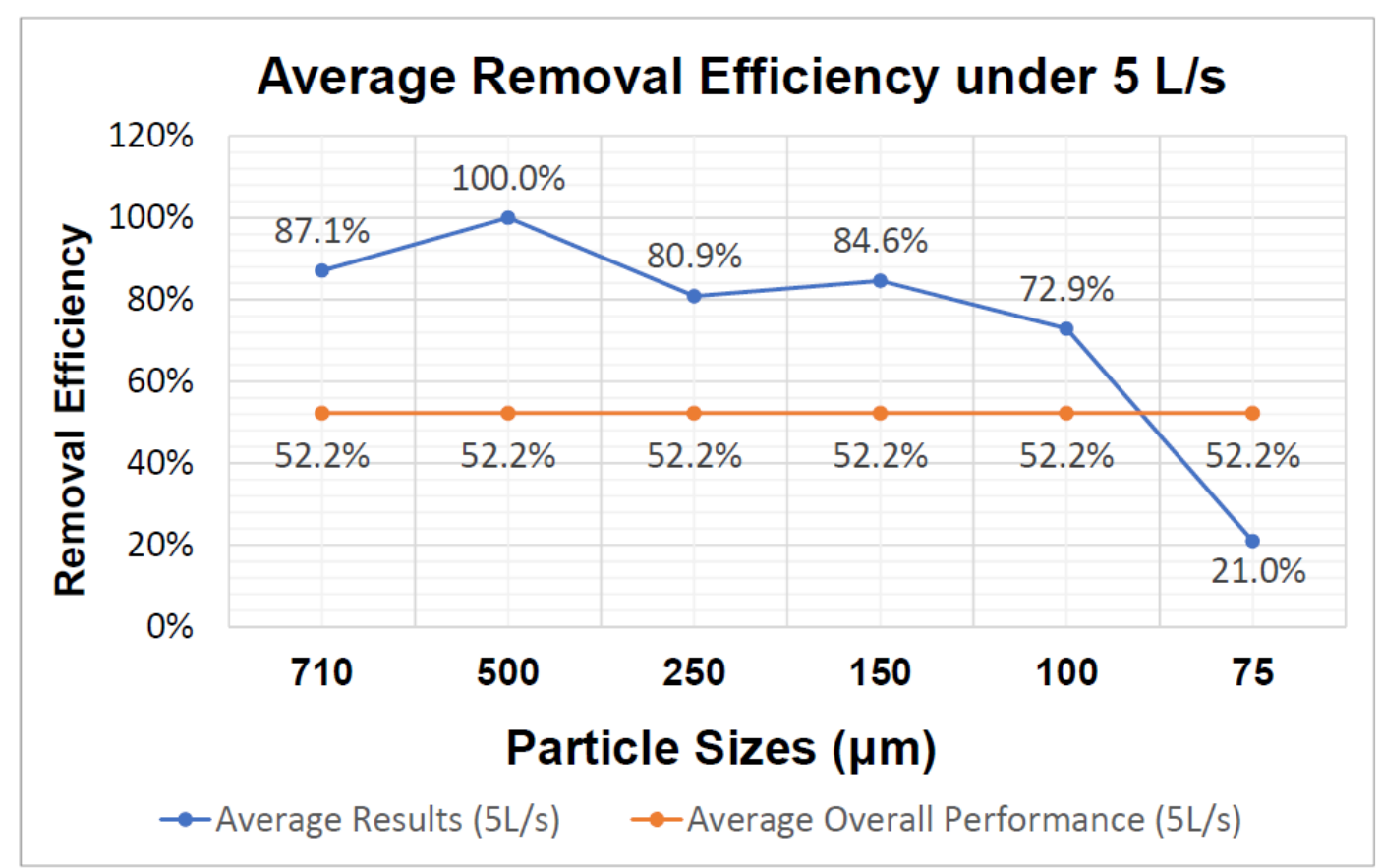

Appendix E. 7 Average Removal Efficiency of Scenarios with 5 L/s Flows

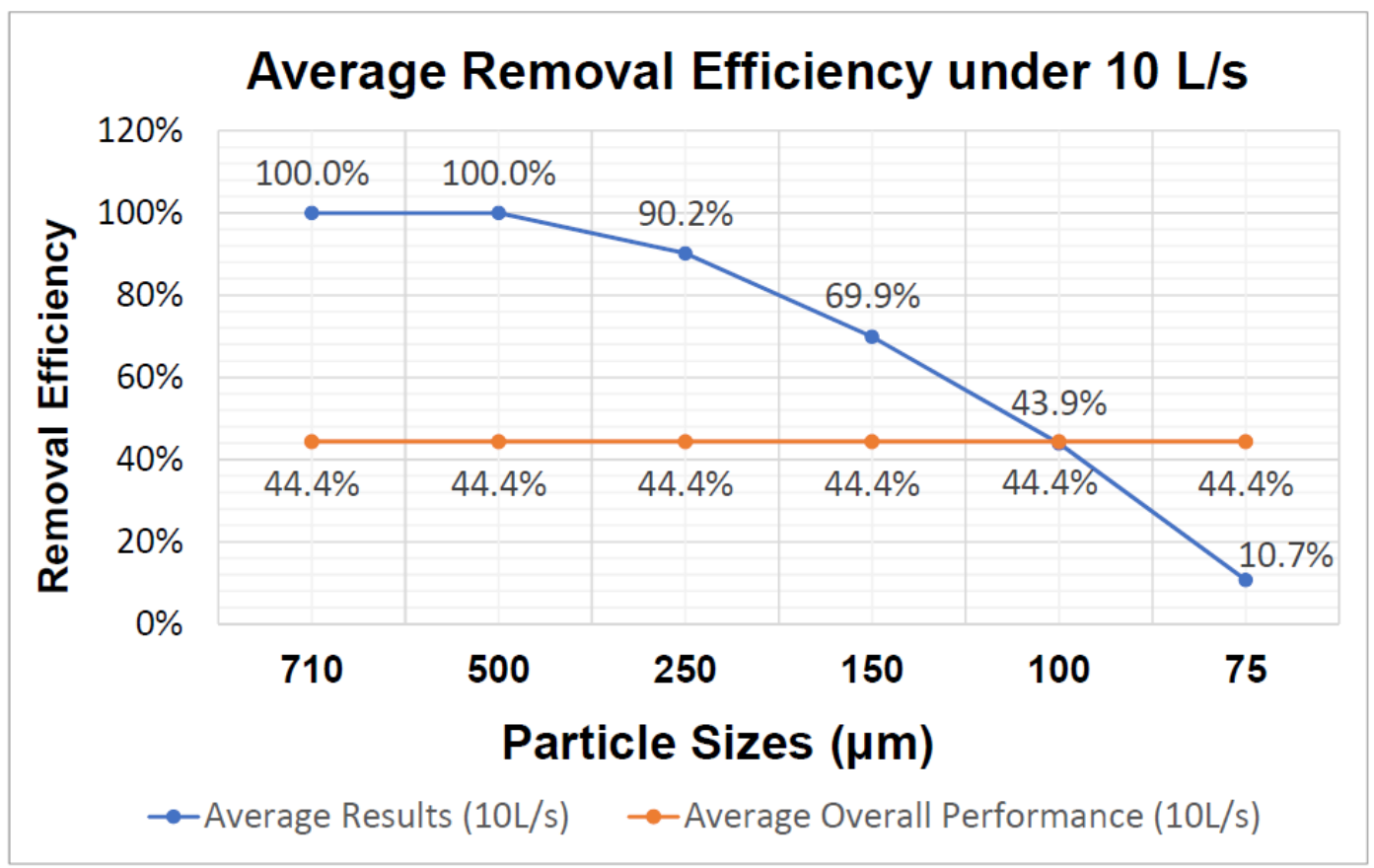

Appendix E. 8 Average Removal Efficiency of Scenarios with $10 \mathrm{~L} / \mathrm{s}$ Flows 


\section{References}

Andoh, R.Y.G., Hides, S.P., Saul, A.J. (2002). Improving Water Quality Using Hydrodynamic Vortex Separator and Screening Systems. Global Solutions for Urban Drainage. 8-13. 10.1061/40644(2002)3.

Andoh, R.Y.G., Smisson, R.P.M. (1994). High Rate Sedimentation in Hydrodynamic Separators. $2^{\text {nd }}$ International Conference on Hydraulic Modeling Development and Application of Physical and Mathmatical Models (Ed. A J Saul), Stratford, UK, 341-358.

Andoh, R.Y.G., Saul, A.J. (2003). The Use of Hydrodynamic Separators and Screening Systems to Improve Water Quality. Water Science and Technology 47(4): 175-183.

Angelakis, A.N. (2017). Urban Waste-and Stormwater Management in Greece: Past, Present, and Future. Water Science and Technology: Water Supply, 17(5), 1386-1399. https://doi.org/10.2166/ws.2017.042

Authority, R. C. (2004). Performance Assessment of Two Types of Oil \& Grit Separator for Stormwater Management in Parking Lot Applications.

Authority, R. C., Canadian, T., Technology, E., \& Program, V. (2014). Procedure for Laboratory Testing of Oil-Grit Separators.

Bian, B., \& Zhu, W. (2009). Particle Size Distribution and Pollutants in Road-deposited Sediments in Different Areas of Zhenjiang, China. Environmental Geochemistry and Health, 31(4), 511-520. https://doi.org/10.1007/s10653-008-9203-8

Chanson, H. (1999). The Hydraulics of Open Channel Flow: An Introduction (Second Edition) Chapter 14: Physical Modeling of Hydraulics. Retrieved May 3, 2020, from https://ebookcentral-proquestcom.ezproxy.lib.ryerson.ca/lib/ryerson/reader.action?docID=288909

Che, W., Liu, Y., Li, J. (2003). Stormwater Quality and Pollution Control in the Domestic and International Urban Areas. Water Supply and Drainage, Vol 29, No 10.

Chou, B. (2015). The Meaning, Approach and Expectation of Sponge City (LID).

Chu, W., \& Ng, F. L. (2000). Upgrading the Conventional Grease Trap Using a Tube Settler. Environment International, 26(1-2), 17-22. https://doi.org/10.1016/S0160-4120(00)00073-8

City of Toronto (2006). Wet Weather Flow Management Guidelines.

Cui, G., Zhang, Q., Zhan, Z., Chen, Y. (2016). Research Progress and Discussion of Sponge City Construction. College of Hydrology and Water Resources, Hohai University, China 
Chui, J. (2000). Control of Oil Spills in Urban Areas. Ryerson University, Canada.

Egarr, D. A., Faram, M. G., O'Doherty, T., \& Syred, N. (2009). Experimental Study of a Hydrodynamic Vortex Separator. Proceedings of the Institution of Mechanical Engineers, Part E: Journal of Process Mechanical Engineering, 223(1), 1-10. https://doi.org/10.1243/09544089JPME225

Elligson, J. C., Mikula, J. B., Clark, S. E., Roenning, C. D., Hafera, J. M., \& Franklin, K. A. (2014). Inclined Plate Settlers to Treat Stormwater Solids. Proceedings of the Water Environment Federation, 2006-6, 5609-5623. https://doi.org/10.2175/193864706783775711

Fan, Z. (2011). Research on Precipitation Trend Analysis and Design Storm of Tianjin City. Construction Department, Tianjin University, China.

Field, R. and O'Connor, T.P. (1996). Swirl Technology: Enhancement of Design, Evaluation and Application. Journal of Environmental Engineering, Vol. 122, No. 8, pp. 741-748.

Fletcher, T. D., Shuster, W., Hunt, W. F., Ashley, R., Butler, D., Arthur, S., ... Viklander, M. (2015). SUDS, LID, BMPs, WSUD and More - The Evolution and Application of Terminology Surrounding Urban Drainage. Urban Water Journal, 12(7), 525-542. https://doi.org/10.1080/1573062X.2014.916314

Frising, T., Noïk, C., \& Dalmazzone, C. (2006). The Liquid/Liquid Sedimentation Process: From Droplet Coalescence to Technologically Enhanced Water/Oil Emulsion Gravity Separators: A Review. Journal of Dispersion Science and Technology, 27(7), 1035-1057. https://doi.org/10.1080/01932690600767098

Fujisaki, K. (2010). Enhancement of Settling Tank Capacity Using a New Type of Tube Settler. Water Science and Technology, 62(6), 1213-1220. https://doi.org/10.2166/wst.2010.841

Galvin, K. P., Zhou, J., \& Walton, K. (2010). Application of Closely SpacedInclined Channels in Gravity sSeparation of Fine Particles. Minerals Engineering, 23(4), 326-338. https://doi.org/10.1016/j.mineng.2009.09.015

Garbon, J. (2018). Canadian Regulatory Guidance for Applications of Oil-Grit Separators in Stormwater Treatment. [PowerPoint Slide]. Retrieved May 14, 2020, from http://www.imbriumsystems.com/Portals/0/webinars/OGS\%20Regulatory\%20Guidance\%2 0Webinar\%20Presentation\%20Feb\%202018.pdf

Gong, Y., Liang, X., Li, X., Li, J., Fang, X., \& Song, R. (2016). Influence of Rainfall Characteristics on Total Suspended Solids in Urban Runoff: A Case Study in Beijing, China. Water (Switzerland), 8(7). https://doi.org/10.3390/w8070278 
Gronowska-Szneler, M. A., \& Sawicki, J. M. (2014). Simple Design Criteria and Efficiency of Hydrodynamic Vortex Separators. Water Science and Technology, Vol. 70, pp. 457-463. https://doi.org/10.2166/wst.2014.245

He, C., \& Marsalek, J. (2009). Vortex Plate for Enhancing Particle Settling. Journal of Environmental Engineering, 135(8), 627-635. https://doi.org/10.1061/(asce)ee.19437870.0000024

Heller, V. (2011). Scale Effects in Physical Hydraulic Engineering Models. Journal of Hydraulic Research, 49(3), 293-306. https://doi.org/10.1080/00221686.2011.578914

Hughes, S.A. (1993). Physical Models and Laboratory Techniques in Coastal Engineering. World Scientific, London. ISBN - 9810215401.

Imbrium Systems Inc. (n.d.). Jellyfish ${ }^{\circledR}$ Filter. Retrieved April 29, 2020, from http://www.imbriumsystems.com/stormwater-treatment-solutions/jellyfish-filter

Jazany, R. A., \& Ghobadi, M. S. (2017). Design Methodology for Inclined Continuity Plate of Panel Zone. Thin-Walled Structures, 113(December 2016), 69-82. https://doi.org/10.1016/j.tws.2016.12.026

Kobus, H., ed. (1980). Hydraulic Modelling. German Association for Water Resources and Land Improvement, Bulletin 7. Parey, Hamburg.

Koloski-Ostrow, A. (2015). The Archaeology of Sanitation in Roman Italy: Toilets, Sewers, and Water Systems. University of North Carolina Press. Retrieved May 14, 2020, from www.jstor.org/stable/10.5149/9781469621296_koloski-ostrow

Kwon, K.-H., Kim, S.-W., Kim, L.-H., Kim, J. H., Lee, S., \& Min, K.-S. (2018). Particle Removal Properties of Stormwater Runoff with a Lab-scale Vortex Separator. Desalination and Water Treatment, Vol. 38, pp. 349-353. https://doi.org/10.5004/dwt.2012.3589

Lake Simcoe Region Conservation Authority \& Simcoe Region Conservation Authority, L. (2013). LSRCA Technical Guidelines for Stormwater Management Submissions. 14.

Li, J. (2018). Water Resource System Analysis: Urban Drainage Design. [Class PowerPoint Slide]. Program of Civil Engineering, FEAS School, Ryerson University, Toronto, Canada

Li, J. (2019). Surface Water Pollution Analysis: Urban Stormwater Impacts. [Lecture Note]. Program of Civil Engineering, FEAS School, Ryerson University, Toronto, Canada

Li, J., Che, W., Liu, Y., Liu, S. (2002). Research on Sedimentation and Particle Size Distribution of Urban Rainwater. Beijing Institute of Civil Engineering and Architecture.

Li, Y. (2009). Development of Design Basis for Hydrodynamic Vortex Separator. New Brunswick Rutgers Graduate school, The State University of New Jersey. 
Lytra, M. (2019). Hydraulics of Lamella Sedimentation - A Study of the Lamella Settlers at Ringsjö Water Treatment Plant, Sweden. Retrived February 9, 2020, from http://lup. lub.lu.se/luur/download?func=downloadFile\&recordOId=8991326\&fileOId=8991327

Mei, C., Liu, J., Wang, H., Xiang, C., \& Zhou, J. (2017). Review on Urban Design Rainstorm. Chinese Science Bulletin, 62(33), 3873-3884. https://doi.org/10.1360/n972016-01295

Metcalf \& Eddy. (2014). Wastewater Engineering: Treatment and Resource Recovery.

Mietzel, T., Klepiszewski, K., \& Weiss, G. (2007). Development and Verification of a General Approach to Describe the Efficiency of Vortex Separators in Combined Sewer Systems. Water Science and Technology, 55(4), 165-173. https://doi.org/10.2166/wst.2007.106

Ministry Of The Environment Ontario. (2003). Stormwater Management Planning and Design Manual. March 2003. In Water Resources.

Ngu, L. H., Law, P. L., Wong, K. K., \& Yusof, A. A. R. (2010). Oil Droplets and Solid Particles Removal Using Circular Separator with Inclined Coalescence Mediums: Comparison between Co-current and Counter-current Flow. Water Science and Technology, 62(5), 1129-1135. https://doi.org/10.2166/wst.2010.407

Novak, P. (1984). Scaling Factors and Scale Effects in Modelling Hydraulic Structures. Symp. Scale effects in modelling hydraulic structures 0(3), 1-5. H. Rouse, ed. Technische Akademie, Esslingen.

Okoth, G., Centikaya, S., Brüggemann, J., \& Thöming, J. (2008). On Hydrodynamic Optimisation of Multi-channel Counter-flow Lamella Settlers and Separation Efficiency of Cohesive Particles. Chemical Engineering and Processing: Process Intensification, 47(1), 90-100. https://doi.org/10.1016/j.cep.2007.08.003

Ontario. (2010). Lake Simcoe Phosphorus Reduction Strategy. Retrieved February 9, 2020, from https://www.ontario.ca/page/lake-simcoe-phosphorus-reduction-strategy\#section-0

Oyegbile, B. A., Hoff, M., Adonadaga, M., \& Oyegbile, B. O. (2017). Experimental Analysis of the Hydrodynamics, Flow Pattern and Wet Agglomeration in Rotor-stator Vortex Separators. Journal of Environmental Chemical Engineering, Vol. 5, pp. 2115-2127. https://doi.org/10.1016/j.jece.2017.04.016

Pitt, R., Robertson, B., Barron, P., Ayyoubi, A., \& Clark, S. (1999). Stormwater Treatment at Critical Areas: The Multi-Chambered Treatment Train (MCTT). Borchardt Conference, a Seminar on Advancements in Water and Wastewater. (March), 507.

Raiyani, J. (2018). Vortex Separator [Powerpoint Slides]. Retrieved on April 10, 2019. https://www.slideshare.net/JIGNESHRAIYANI2/vortex-separator-to-separate-differentdensity-fluid 
Saady, N. M. C. (2012). Utilizing Settling Tests to Design a Conventional Upflow Settling Tank Modified with Inclined Plates. Water Science and Technology, 66(4), 858-864. https://doi.org/10.2166/wst.2012.260

Salem, A. I., Okoth, G., \& Thöming, J. (2011). An Approach to Improve the Separation of Solidliquid Suspensions in Inclined Plate Settlers: CFD Simulation and Experimental Validation. Water Research, 45(11), 3541-3549. https://doi.org/10.1016/j.watres.2011.04.019

Sansalone, J. J., \& Cristina, C. M. (2004). First Flush Concepts for Suspended and Dissolved Solids in Small Impervious Watersheds. Journal of Environmental Engineering, 130(11), 1301-1314. https://doi.org/10.1061/(asce)0733-9372(2004)130:11(1301)

Sansalone, J. J., Koran, J. M., Smithson, J. A., \& Buchberger, S. G. (2002). Physical Characteristics of Urban Roadway Solids Transported during Rain Events. Journal of Environmental Engineering, 124(5), 427-440. https://doi.org/10.1061/(asce)07339372(1998)124:5(427)

Sansalone, J., Lin, H., \& Ying, G. (2009). Experimental and Field Studies of Type I Settling for Particulate Matter Transported by Urban Runoff. Journal of Environmental Engineering, 135(10), 953-963. https://doi.org/10.1061/(asce)ee.1943-7870.0000078

Sansalone, J., Ying, G., \& Lin, H. (2009). Distribution of Metals for Particulate Matter Transported in Source Area Rainfall-Runoff. Journal of Environmental Engineering, 136(2), 172-184. https://doi.org/10.1061/(asce)ee.1943-7870.0000139

Sarkar, S., Kamilya, D., \& Mal, B. C. (2007). Effect of Geometric and Process Variables on the Performance of Inclined Plate Settlers in Treating Aquacultural Waste. Water Research, 41(5), 993-1000. https://doi.org/10.1016/j.watres.2006.12.015

Schlieper, L., Chatterjee, M., Henschke, M., \& Pfennig, A. (2004). Liquid-Liquid Phase Separation in Gravity Settler with Inclined Plates. AIChE Journal, 50(4), 802-811. https://doi.org/10.1002/aic.10075

Science. (2013). China's Rapid Urbanization. 18 October, Vol 342.

Sedimentation (Water Treatment). (n.d.). In Wikipedia. Retrieved on April 8, 2019. https://en. wikipedia.org/wiki/Sedimentation_(water_treatment)

Shen, Z., Liu, J., Aini, G., \& Gong, Y. (2016). A Comparative Study of the Grain-size Distribution of Surface Dust and Stormwater Runoff Quality on Typical Urban Roads and Roofs in Beijing, China. Environmental Science and Pollution Research, 23(3), 2693-2704. https://doi.org/10.1007/s11356-015-5512-5 
Smith, B. T., Halperin, J., Darzins, A., \& Davis, R. H. (2013). Enhanced Sediment Flow in Inclined Settlers via Surface Modification or Applied Vibration for Harvesting Microalgae. Algal Research, 2(4), 369-377. https://doi.org/10.1016/j.algal.2013.05.004

Sullivan, R., Cohn, M., Coomes, J., Smission, B., Coombes, J., Smission, B. (1972). The Swirl Concentrator as a Combined Sewer Overflow Regulator Facility. Report No: EPA-R2-72008, US Environmental Protection Agency, Washington, DC.

Sullivan, R.H., Cohn, M.M., Ure, J.E., Parkinson, F., Galiana, G., Boericke, R.R.; Koch, C., and Zielinski, P. (1978). The Swirl Primary Separator: Development and Pilot Demonstration. Report No: EPA-600/2-78-122, US Environmental Protection Agency, Cincinnati, Ohio.

Sun, Y., Wei, X., Pomeroy, C. (2011). Review of Current Research and Future Directions of Low Impact Development Practices for Storm Water. Department of Civil Engineering, North-West Agriculture and Forest Technology University, China \& Department of Civil and Environmental Engineering, University of Utah, USA. Advance in Water Science, 22(2), 287-293.

Takayanagi, E., Abe, T., Taruya, T., Fukui, S. (1997). Introduction of Plate Sellter for Combined Sewer Overflow Treatment. Sewage Works Bereau, Osaka City Government.

Tarpagkou, R., \& Pantokratoras, A. (2014). The Influence of Lamellar Settler in Sedimentation Tanks for Potable Water Treatment - A Computational Fluid Dynamic Study. Powder Technology, 268, 139-149. https://doi.org/10.1016/j.powtec.2014.08.030

Task Committee on Hydraulic Modeling, Environmental and Water Resources Institute, ASCE R. Ettema. (2000). Hydraulic Modeling - Concepts and Practice - ASCE Manuals and Reports on Engineering Practice (MOP) No. 97. American Society of Civil Engineers (ASCE). Retrieved from https://app.knovel.com/hotlink/toc/id:kpHMCP000D/hydraulicmodeling-concepts/hydraulic-modeling-concepts

Thompson, P. \& Galvin, K. (2002). An Empirical Description for the Classification in an Inclined Counter-flow Settler. Minerals Engineering, 10(1), 97-109. https://doi.org/10.1016/s0892-6875(96)00134-3

Toronto and Region Conservation Authority - Stormwater Assessment Monitoring and Performance (SWAMP) Program. (2004). Performance Assessment of Two Types of Oil \& Grit Separator for Stormwater Management in Parking Lot Applications.

Toronto and Region Conservation Authority. (2014). Procedure for Laboratory Testing of OilGrit Seperators.

Tyson Ochsner. (2013, October 23). Sediment Deposition and Stokes' Law [Video]. YouTube. https://www.youtube.com/watch?v=xGNFimTv0mE

U.S. Environmental Protection Agency. (2003). Framework of cumulative risk assessment. Risk Assessment Forum, Washington, DC. EPA/630/P-02/001F. 1-47. 
Wang, Q., Zhang, Q., Dzakpasu, M., Lian, B., Wu, Y., \& Wang, X. C. (2018). Development of an Indicator for Characterizing Particle Size Distribution and Quality of Stormwater Runoff. Environmental Science and Pollution Research, 25(8), 7991-8001. https://doi.org/10.1007/s11356-017-1074-z

Wikipedia. (2019). Lamella Clarifier. Retrieved February 9, 2020, from https://en.wikipedia.org/wiki/Lamella_clarifier

Wikipedia. (2019). Similitude (Model). Retrieved May 3, 2020, from https://en.wikipedia.org/wiki/Similitude_(model)

Wikipedia. (2020). Stormwater. Retrieved February 9, 2020, from https://en.wikipedia.org/wiki/Stormwater

Wikipedia. (2020). Water Pollition. Retrieved February 9, 2020, from https://en.wikipedia.org/wiki/Water_pollution

Yalin, M.S. (1971). Theory of Hydraulic Models. Macmillan, London.

Yu, K., Li, D., Yuan, H., Fu, W., Qiao, Q., \& Wang, S. (2015). “Sponge City”: Theory and Practice. Planning Studies, 39(6), 26-36.

Yufen, R., Xiaoke, W., Zhiyun, O., Hua, Z., Xiaonan, D., \& Hong, M. (2008). Stormwater Runoff Quality from Different Surfaces in an Urban Catchment in Beijing, China. Water Environment Research, 80(8), 719-724. https://doi.org/10.2175/106143008x276660

Zhang, W., \& Li, T. (2015). Particle Size Distributions in Combined Sewer Overflows in a High-intensity Urban Catchment in Shanghai, China. Desalination and Water Treatment, 56(3), 655-664. https://doi.org/10.1080/19443994.2014.942703

Zhang, Y. (2018). Performance Test of a Rotary Lamella Settler and its ICEM-CFD Mesh Generation. School of Water Conservancy and Environment, Zhengzhou University.

Zhang, Z., Chen, Y., Wang, P., Shuai, J., Tao, F., \& Shi, P. (2014). River Discharge, Land Use Change, and Surface Water Quality in the Xiangjiang River, China. Hydrological Processes, 28(13), 4130-4140. https://doi.org/10.1002/hyp.9938 